\title{
HYDRODYNAMIC ASPECTS OF BOILING HEAT TRANSFER (thesis)
}

By

Novak Zuber

June 1959

$\cdots$

\section{Research Laboratory}

(Los Angeles) and Ramo-Wooldridge Corporation University of California.

Los Angeles, California

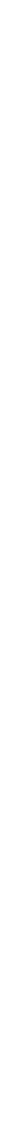




\section{LEGAL NOTICE}

This report was prepared as an account of Government sponsored work. Netther the United States, nor the Commisston, nor any person acting on behalf of the Commiseton:

A. Makes any warranty or representation, expressed or implled, with respect to the accuracy, completeness, or usefulness of the information contained in this report, or that the use of any information, apparatus, method, or procese disclosed in this report may not infringe privately owned righte; or

B. Assumes any liabllities wh reapect to the use of, or for damages resulting from the use of any Information, apparatus, method, or process dieclosed in this report.

As used in the above, "person acting on behalf of the Commisalon" Includes any employee or contractor of the Commission, or employee of such contractor, to the extent that such employee or contractor of the Commission, or employee of euch contractor prepares, disseminates, or provides access to, any information pursuant to his employment or contract with the Commission, or his employment with such contractor.

This report has been reproduced directly from the best available copy.

Printed in USA. Price $\$ 2.00$. Avallable from the Office of Technical Services, Department of Commerce, Washington 25, D. C.

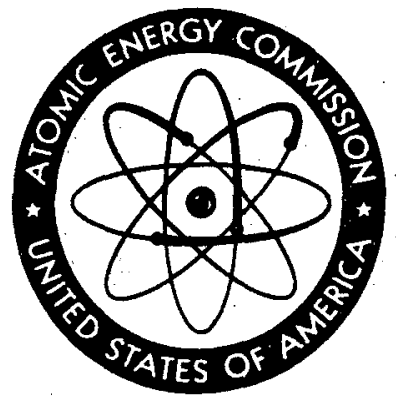




\section{DISCLAIMER}

This report was prepared as an account of work sponsored by an agency of the United States Government. Neither the United States Government nor any agency Thereof, nor any of their employees, makes any warranty, express or implied, or assumes any legal liability or responsibility for the accuracy, completeness, or usefulness of any information, apparatus, product, or process disclosed, or represents that its use would not infringe privately owned rights. Reference herein to any specific commercial product, process, or service by trade name, trademark, manufacturer, or otherwise does not necessarily constitute or imply its endorsement, recommendation, or favoring by the United States Government or any agency thereof. The views and opinions of authors expressed herein do not necessarily state or reflect those of the United States Government or any agency thereof. 


\section{DISCLAIMER}

Portions of this document may be illegible in electronic image products. Images are produced from the best available original document. 


\section{UNIVERSITY OF CAIIFORNIA, LOS ANGELES}

Hydrodynamic Aspects

of Boiling Heat Transfer

A dissertation submitted in partial satisfaction of the requirements for the degree Doctor of Philosophy

in Engineering

by

Novak Zuber

June, 1959 


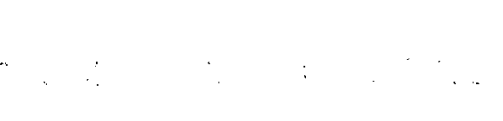


TABLE OF CONTENTS

Chapter

Page

INTRODUCTION . . . . . . . . . . . . I

I A REVIEW OF NUCLEATE BOILING . . . . . 6

Nucleation from a Solid Surface . . . . 7

Bubble Generation .......... 9

The Dynamics of a Bubble Departing from

a Horizontal, Heated Surface . . . . 15

The Relation Between Heat Flux Density,

Surface Roughness and Temperature in

Nucleate Boiling . . . . . . . 24

II THE PROBLEM OF BUBBLE GROTTH . . . . . 34

The Dynamics of Vapor. Bubbles in a Uniform

Temperature Field - Historical Background 34

The Growth of a Vapor Bubble in a Non-Uniform

The Bubble Growth in ilquids at Saturation Temperature ........... 47

Maximum Bubble Diameter and the Thiclmess of the Superheated Liquid Film . . . . 51

The Thickness of the Superheated Liquid Film

Implications and Limitations of the Model 55

Dynamics of a Vapor Bubble in a Subcooled

liquid .:............. 61

III HYDRODYNAMIC ASPECTS OF NUCLEATE BOILING 77

The Similarity Between Bubble. Formation at an Orifice and Bubble Formation in Nucleate Boiling Bubble Emission and the

Value of the Product: Bubble Diameter

Times Frequency in Nucleate Boiling .

A Possible Relation Between the Diameter of a Quasi-Static Bubble and the

Diameter of a Cavity ......... 
III (Continued)

On the Similarity Between Nucleate Boiling and Flow of Gas Bubbling Through a Perforated Surface . . . . 89 The Phenomenon of "Flooding" ...... 93 Previous Empirical Correlations of the Critical Heat Flux . . . . . . . 97 Nucleate Boiling and the Region of the Critical Heat Flux . . . . . . . . 106 Nucleate Boiling Heat Transfer - Methods of Analysis . . . . . . . . . 112

IV HYDRODINAMIC ASPECTS OF TRANSITIONAI BOILING

Stability of a Plane Interface Mathematical Formulation . . . . . 119 Stability of a Plane Interface Esperimental Results . . . . . . 123 Westwater and Santangelo's Description of Transitional Boiling . . . . . . 127 The Hydrodynamic Instability of Transitional Boiling . . . . . . . . 129

$V$ THE MINTMUM HEAT FLUX DENSITY IN TRANSITIONAI BOILING FROM A HORIZONTAL SURFACE

Minimum Heat Flux Density - Formulation and Solution of the Problem ..... 138 The Minimum Heat Flux Density - Comparison of Analytical and Experimental Results . 146

VI THE CRITICAL HEAT FLUX IN BOILING FROM A HORIZONTAL SURFACE . . . . . . . 149

The Critical Heat Flux - Formulation and Solution of the Problem . . . . . 150 The Critical Heat Flux - Comparison of Theoretical with Experimental Results . 157 The Critical Heat Flus in Pool Boiling of a Subcooled Liquid . . . . . . 164 
Chapter

Page

CONCLUSIONS AND SUMMARY

173

BIBLIOGRAPHY

179

APPENDIX A

189 
TABLE OF FIGURES

F1gure

Page

I A Typical Curve of Heat Flux Density versus Surface Temperature in Boiling 2

I-1. Nucleation from a Cavity: Liquid Wets the Solid ........ 10

I-2. Nucleation from a Cavity: Liquid Does Not Wet the Solid ..... 10

I-3. Nucleation from a Conical Cavity . . 13

I-4. Deformation of a Bubble Departing from a Heated Horizontal Surface According to Jakob ........... 18

I-5. Types of Vapor Bubbles Departing from a Horizontal Heated Surface as Reported by Yamagata and Nishikawa ..... 21

I-6. The Effect of Different Amounts of Roughness on the Heat Transfer in Nucleate Boiling . . . . . . 29

II-1. Bosnjakovic's Analytical hodel of a Bubble Growing in a Uniformly Superheated Liquid .........

II-2. Jakob's Analytical Model of a Bubble Growing in a Non-Uniform Temperature Field

II-3. Comparison of Equation II-17 with Zmola's Experimental Data for Bubbles in Pool Boiling of Water at Saturation Temperature ....... 50

II-4. The Probable Bubble Form and the Approximation of the Model ... 57 
Figure

II-5. Ellion's Experimental Data for Bubbles Growing and Collapsing in Subcooled

Water ... . . . . . . . . .

II-6. Ellion's Experimental Data for Bubbles Groving and Collapsing in subcooled

II-7. Ellion's Experimental Data for Bubbles Growing and Collapsing in subcooled

II-8. Ellion's Experimental Data for Bubbles Growing and Collapsing in Subcooled Wat:er

II-9. Comparison of Equations II-30 and II-33 with Ellion's Experimental Data Shown on Figure II-5.

II-10. Comparison of Equations II-30 and II-33 with Ellion's Experimental Data Shorm on Figure II-6.

II-11. Comparison of Equations II-30 and II-33 with Ellion's Experimental Data Shorn on Figure II-7.

II-12. Comparison of Equations II-30 and II-33 with Ellion's Experimental Data Shown on Figure II-8.

III-1. The Relation Between the Frequency of Bubble Enission and the Diameter of $a$ Bubble Departing from a Horizontal Surface

III-2.

The Relation Between the Superficial Gas Velocity, and the Pressure Drop Across a Porous Plate as a Function of the Pore Size ............

III-3. Correlation of Data for Various Liquids at the Critical Heat Flux Density in Pool Boiling 
III-4. Schematic Representation of the Process of Nucleate Boiling ....... 108

IV-1. Schematic Representation of the Process of Transitional Boiling ...... 130

VI-1. Comparison of Predicted with Experimentally Determined Critical Heat Flux Densities in Pool Boiling for Water at Saturation Temperature

VI-2. Comparison of Predicted with Experimentally Determined Gritical Heat Flux Densities in Pool Boiling for Ethyl Alcohol at Saturation Temperature . . 163

VI-3. Comparison of Predicted with Experimentalis Determined Gritical Heat Transfer Rates in Pool Boiling of Subcooled Liquids

VI-4. Comparison of Predicted with Experimentally Determined Critical Heat Transfer Rates in Pool Boiling of Subcooled Water

VI-5. Comparison of Predicted with Experimentally Determined Critical Heat Transfer Rates in Pool Boiling of Subcooled Ethyl Alcohol at Various Pressures . . . . 171 


\section{NOMENCLATURE}

$$
\begin{aligned}
& \text { a = thermal diffusivity } \\
& \text { A = area } \\
& \text { b }=\text { a numerical constant equal to } I \text { or } \pi / 2 \\
& \text { c = specific heat at constant pressure } \\
& \text { C = propagation velocity of a disturbance } \\
& D_{c} \quad=\text { diameter of a nucleating cavity } \\
& \mathrm{D}_{\mathrm{d}}=\text { diameter of a bubble departing from a } \\
& \text { horizontal surface } \\
& \mathrm{D}_{\mathrm{m}} \quad=\text { maximum bubble diameter } \\
& \text { f }=\text { Erequency } \\
& \text { g = acceleration due to gravity } \\
& \text { h = heat transfer coeficient } \\
& \mathrm{H}_{\mathrm{O}}=\text { amplitude of a disturbance }
\end{aligned}
$$

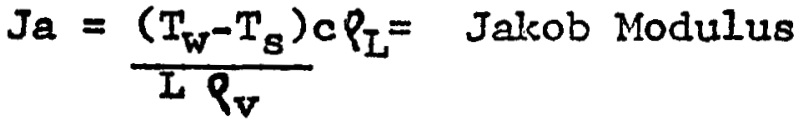

$$
\begin{aligned}
& I_{0}=\text { modified Bessel function of the first kind } \\
& K_{0}=\operatorname{modified~Bessel~function~of~the~second~}_{\text {lcind }} \\
& \text { k = thermal conductivity } \\
& \mathrm{I}=\text { latent heat of vaporization } \\
& \text { m = wave number } \\
& \text { M = vapor mass flow rate per unit area } \\
& \mathrm{n}=\text { number of bubbles }
\end{aligned}
$$




$$
\begin{aligned}
& \text { P }=\text { pressure } \\
& q \text { = heat flux density from solid to liquid } \\
& q_{b}=\text { heat flux density from bubble interface } \\
& \text { to bulk liquid } \\
& q_{c}=\text { critical heat flux } \\
& q_{m}=\text { minimum heat flus in transitional boiling } \\
& r_{0} \quad=\text { radius of a surface cavity } \\
& \mathrm{R} \quad=\text { bubble radius } \\
& \mathrm{R}_{\mathrm{tm}}=\text { maximum bubble radius in subcooled boiling } \\
& t_{c}=\begin{array}{l}
\text { contact time, i.e., the time needed to } \\
\text { superheat the liquid }
\end{array} \\
& t_{\mathrm{d}}=\text { the time needed for a bubble to reach } \\
& \text { the diameter } \mathrm{D}_{\mathrm{d}} \\
& t_{m}=\text { the time needed for a bubble to reach } \\
& \text { the radius } R_{m} \\
& T_{L} \quad=\text { temperature of the bulk liquid } \\
& T_{0}=\text { temperature of the superheated liquid } \\
& T_{\mathbf{s}} \quad=\text { saturation temperature } \\
& T_{W}=\text { temperature of the solid, i.e., of the } \\
& \text { wall and therefore of the liquid in } \\
& \text { contact with it } \\
& T=T_{0}-T_{s}=T_{W}-T_{S}=\begin{array}{l}
\text { liquid superheat temperature } \\
\text { difference }
\end{array} \\
& u_{v}=\text { velocity of the vapor } \\
& U_{\mathrm{L}} \quad=\text { velocity of the liquid } \\
& \mathrm{U}=\text { velocity of rise of deformed bubbles } \\
& \mathrm{x}_{\mathrm{s}}=\text { thiclness of the superheated liquid film } \\
& \text { adjacent to the heating surface }
\end{aligned}
$$




$$
\begin{aligned}
& \alpha=\text { conical angle of a nucleating cavity } \\
& \delta=\text { thickness } \alpha \text { the thermal boundary layer } \\
& \lambda=\text { wave length } \\
& \eta=\text { disturbance of a plane interface } \\
& y=\text { disturbance of a cylindrical interface } \\
& \omega=\text { angular frequency } \\
& \rho=\text { density } \\
& \sigma=\text { surface tension } \\
& \theta=\text { contact angle }
\end{aligned}
$$

Subscripts

$$
\begin{aligned}
& v \quad=\text { vapor } \\
& \mathrm{I} \quad=\text { liquid }
\end{aligned}
$$




\section{ACKONOWLEDGEMENTS}

The author wishes to express his deep gratitude to Professor Myron Tribus without whose encouragement and support this investigation would not have been completed.

This study was initiated while the writer was associated with the Department of Engineering, University of California, Los Angeles; 1t was completed after he became a staff member of the Research Laboratory of the Ramo-Wooldridge Corporation. The author is grateful to the Department of Engineering and to the Research Laboratory for providing an atmosphere conducive to research. 
Mojim dobrim roditeljima - tamo daleko, and to Corinne close by.

$\mathrm{Na}$ Vidovdan 1959

xiii 


\section{ABSTRACT}

An analytical expression is presented which permits the prediction of the critical heat flux density in pool bolling of saturated or subcooled liquids. The theoretical results are in good agreement with experimental data.

The hydrodynamic characteristics of transitional bolling from a horizontal surface are analyzed; the phenomenon is interpreted in terms of Taylor instability. It is shown that the minimum heat flux is limited by the effect of Taylor Instability only; an equation is derived which permits the prediction of this heat flux density.

The hydrodynamic characteristics of nucleate boiling from a horizontal surface are analyzed. The similarity between bubble formation at an orifice and bubble formation in nucleate boiling is investigated. This similarity indicates a relation between the diameter of a quasl-static bubble departing from the surface and the radius of the nucleating cavity. An equation is derived also for the product bubble diameter times frequency of bubble enission; the predicted values are in good agreement with experimental data of Jakob. It is possible thus to estimate the maximum frequency of the 
release of single bubbles in nucleate boiling. The similarity between nucleate boiling and the process of gas bubbling from a porous surface is discussed. The similarity indicates that the critical heat flux is a hydrodynamic phenomenon known as "flooding".

The nucleation from a solid surface is reviewed. The analysis of available experimental data indicates that the diameter of a nucleating cavity can be related to the heat flus density and to the superheat temperature difference. Further experimental investigations are needed.

The problem of bubble growth is discussed. It is pointed out that the original formulation of the problem as given by Bosnjaleovic and Jalkob contains the essential features of the problem. The theory of Bosnjakovic and Jakob is extended to include the effects of a non-uniform temperature field. 


\section{INTRODUCTION}

Developments in nuclear reactors and rocket engines where exceedingly high heat transfer rates occur in comparatively small areas, have focused attention on boiling as a mode of transferring heat at high flux densities. To attain these high heat transfer rates by forced convection would require excessively high velocities with resulting high pressure drops. With nucleate boiling, however, they can be reached at much lower bulk velocities. For this reason extensive experimental and theoretical studies of this phenomenon are conducted in this country and abroad.

The phenomenon is complex because three different regimes exist: nucleate, transition and film boiling. The change from one regime to another is accompanied by marked changes in the hydrodymamic and thermal state of the system. These regimes are illustrated in Fig ure I. When the temperature of the heating surface is below the fluid saturation temperature heat is transferred by convection, forced or natural, depending on the system. This non-boiling region ( $A B$ ) has been extensively investigated and equations have been derived which permit the prediction of heat transfer rates. 


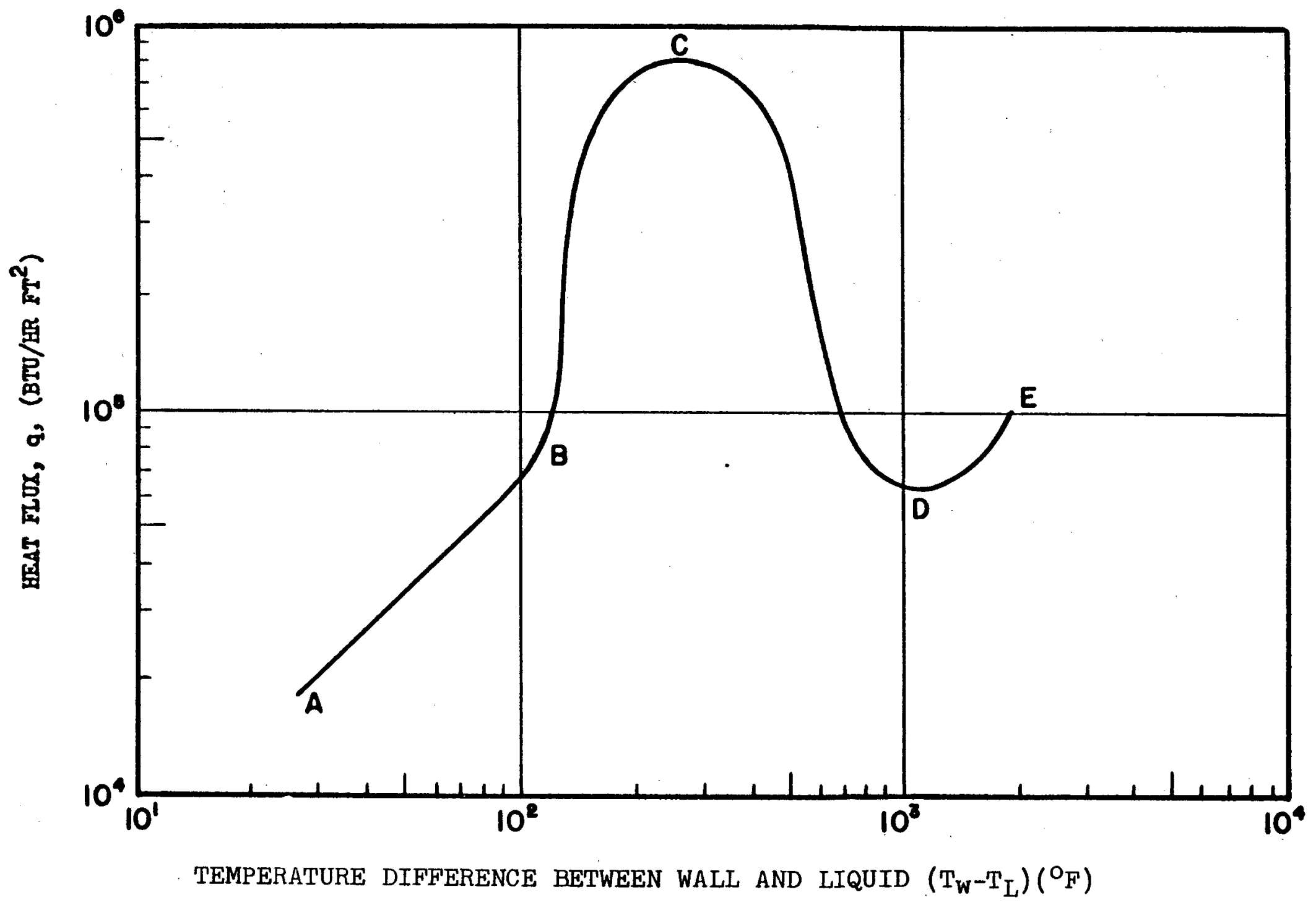

FIGURE I. A TYPICAL CURVE OF HEAT FLUX DENSITY VERSUS SURFACE TEIVIPERATURE IN BOILING 
Nucleate boiling ( $B C)$ starts when the temperature of the surface exceeds the saturation by a few degrees. Adjacent to the surface a thin layer of superheated liquid is formed in which bubbles nucleate and grow from some preferred spots on the surface. The thermal resistance of this superheated liquid film is greatly reduced by the agitation produced by the bubbles. An increase of the wall temperature is accompanied by a large increase of the bubble population causing in turn a sharp increase of the beat flux. However, as the temperature increases, bubbles become so numerous that their motions intereact. Under these conditions the nucleate heat flux reaches its peak. If the temperature is further increased transition boiling begins. Westwater and Santangello (I) have found that in this region (CD) no liquid-solid contact exists. The surface is blanketed by an unstable, irregular film of vapor which is in violent motion. In transition boiling an increase of temperature is followed by a decrease of heat flux until a minimum value is reached at which point film boiling starts. This new regime is characterized by an orderly discharge of large bubbles with a regular frequency and at regular intervals. In the film bolling region the heat flux increases with an increase of temperature but at a much slower rate than in nucleate 
bolling. Consequently, at high heat transfer rates the temperature of the heating surface can exceed the melting temperatire and "burnout" $\propto$ curs. It is of great practical interest to operate in the nucleate region becavse of the favorable heat transfer. The problem is to avo id the "burnout" phenomenon.

The temperatures of the wall in nucleate boiling are quite low, for example, with boiling water the temperature of the surface at the point $C$ exceeds the fluid saturation temperature by about $50^{\circ} \mathrm{F}$ at 14.6 psia and by only $10^{\circ} \mathrm{F}$ at 2000 psia. Therefore, in many designs the exact surface temperatures are of secondary importance. This is especially tmue for constant heatinput systems such as a nuclear reactor. The essential information needed by a desigmer is the limft to the heat transfer rates given by the critical heat flux, i.e., by the flux corresponding to point $C$. The temperature at point $C$ is relatively unimportant. An investigation of the conditions leading to this maxdmum heat flux is therefore of practical and theoretical interest. Such a study, as applied to a horizontal plate, is reported in this dissertation. The study is concerned with determining the limiting hydrodynamic conditions. which characterize nucleate and transitional 
bolling. The critical heat flux (point $C$ ) and the minimm heat flux (point D) are derived from these hydrodynamic Iimits.

An understanding of the conditions leading to the changes in the regimes of bolling requires an understanding of the processes which characterize each regime separately. Because of this, both nucleate and transitional boiling are investigated and discussed. The dissertation is divided into six chapters according to the problem under consideration. Results of experimental investigation of nucleate boiling are discussed in Chapter I, which is, therefore, a review section. The problem of bubble growth is discussed in this dissertation because nucleate boiling is characterized by the action of vapor bubbles. In Chapter II, the bubble growth theory of Bosnjakovic and Jakob is extended to include the effect of a non-uniform temperature field. In Chapter III, the hydrodynamic aspect of nucleate boiling is analyzed, wherens the hydrodynamic aspect of transitional boilfing is investigated in Chapter IV. The minimum and the critical heat flux are analyzed in Chapters $V$ and VI respectively. 


\section{CHAPTER I}

\section{A REVIEW OF NUCLEATE BOILING}

The first systematic investigation of nucleate bolling was undertaken by Jakob, Fritz and Linke, the results of which were reported in a series of outstanding papers $(2,3,4,5,6)$. The high heat transfer rates in nucleate boiling were attributed to bubbles which induce locally a strong agitation of the liquid near the heating surface. The effect of bubble agitation on the heat flux was further investigated and confirmed by Gunther and Kreith (7) and by Rohsenow and Clark ( 8 ).

Jakob and Fritz (2) noted that the high heat transfer rates, which are caused by local flow oscillations that exist in the vicinity of growing and rising bubbles, can be induced also by liquid jets flowing in between rising bubble columns and impinging on the heating surface. The total heat flux from the heating surface to the liquid is the sum of such local heat transfer rates. Consequently, in order to gain an understanding of nucleate boiling it is necessary to understand the local processes which are associated with single bubbles. It is necessary thus to investigate 
three aspects of the problem: the nucleation of a bubble, the growth of a bubble, and finally, the hydrodynamics of a bubble departing from the heating surface. These three aspects of the problem, which were investigated by Jakob and co-workers as well as by other investigators, w1ll be discussed in the sections which follow.

I-1. Nucleation from a Solid Surface

The effect of a surface on the process of nucleation will be discussed in some detail because, as it will be seen in the sections which follow, the conditions of the surface determine the temperature at which the bubble will generate and, thus, determine the subsequent history of a growing bubble.

It is experimentally observed that in bolling liquids bubbles nucleate at liquid superheat temperatures which are considerably less than those which would be predicted by classical nucleation theories. It 1s known, also, that bubbles originate from specific nucleating centers located on the heated surface. The question of whether these nucleating centers are roughness projections or cavities in the surface was examined in detail by Bankoff (9). By comparing the theoretical superheat temperatures which would be required to generate a bubble from a flat or projecting surface, with the superheats which are observed in experiments, Bankoff concluded 
that only pits or scratches which contain gas or vapor are possible sites for nucleation. These deductions were conclusively verified by the experimental results of Clark, Strenge and Westwater (10).

The nucleation process fron a cavity was investigated by Jakob and co-workers $(2,3,4,5)$; by Dzhandzhgava (11); Nesis (12); Courty and Foust (13, 14); Ellion (15); Bankoff (9); Clark, Strenge and Westwater (10); and by Griffith and Wallis (16). The problem can be resolved into two parts: one, an investigation of the conditions necessary to entrap gas and/or vapor into surface capillaries (pits or scratches) and, two, an investigation of conditions which would permit the release of this vapor in the form of a bubble.

The process of entrapment of a gas and/or vapor into a cavity and the related problem of investigating whether this cavity will remain stable in time, i.e., whether or not the entrapped gas will diffuse and whether the entrapped vapor can condense, are of importance in an analysis of transient boiling and of boiling hysteresis. Since these latter two processes are not the topic of the present investigation, the entrapment and the stability of a cavity will not be discussed here. The reader is referred to the papers by Courty (13), Benleoff $(17,18)$ and by Sabersley and 
Gates (19) for a discussion and investigation of this problem. An analysis of transient boiling will be presented in a future report where the entrapment process will be taken up also.

We shall proceed with the discussion of the conditions which permit the release of a gas or vapor from a cavity, a problem which was first analyzed by Courty and Foust (13, 14) in this country and by Nesis (12) in Russia.

I-2. Bubble Generation from a Cavity

Consider an amount of gas or vapor which is entrapped in a cylindrical capillary with vertical walls. shown in Figures $I-1$ and I-2, which are reproduced from Nesis' paper (12). When the liquid wets the solid, i.e., when the contact angle $\theta$, is less than $90^{\circ}$ (Figure I-1), the pressure in the cavity is given by:

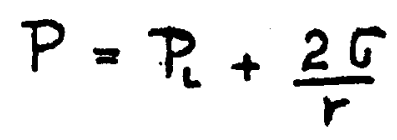

where $r$ is the radius of the curvature of the interface and $P_{I}$ is the pressure in the liquid. The maximum pressure in the cavity occurs when the radius, $r$, is minimum. In expanding from stage I to stage VI, the minimum curvature occurs when the interface turns around the comer. The minimum value of $r,\left(r_{\min }=r^{*}\right)$ is 


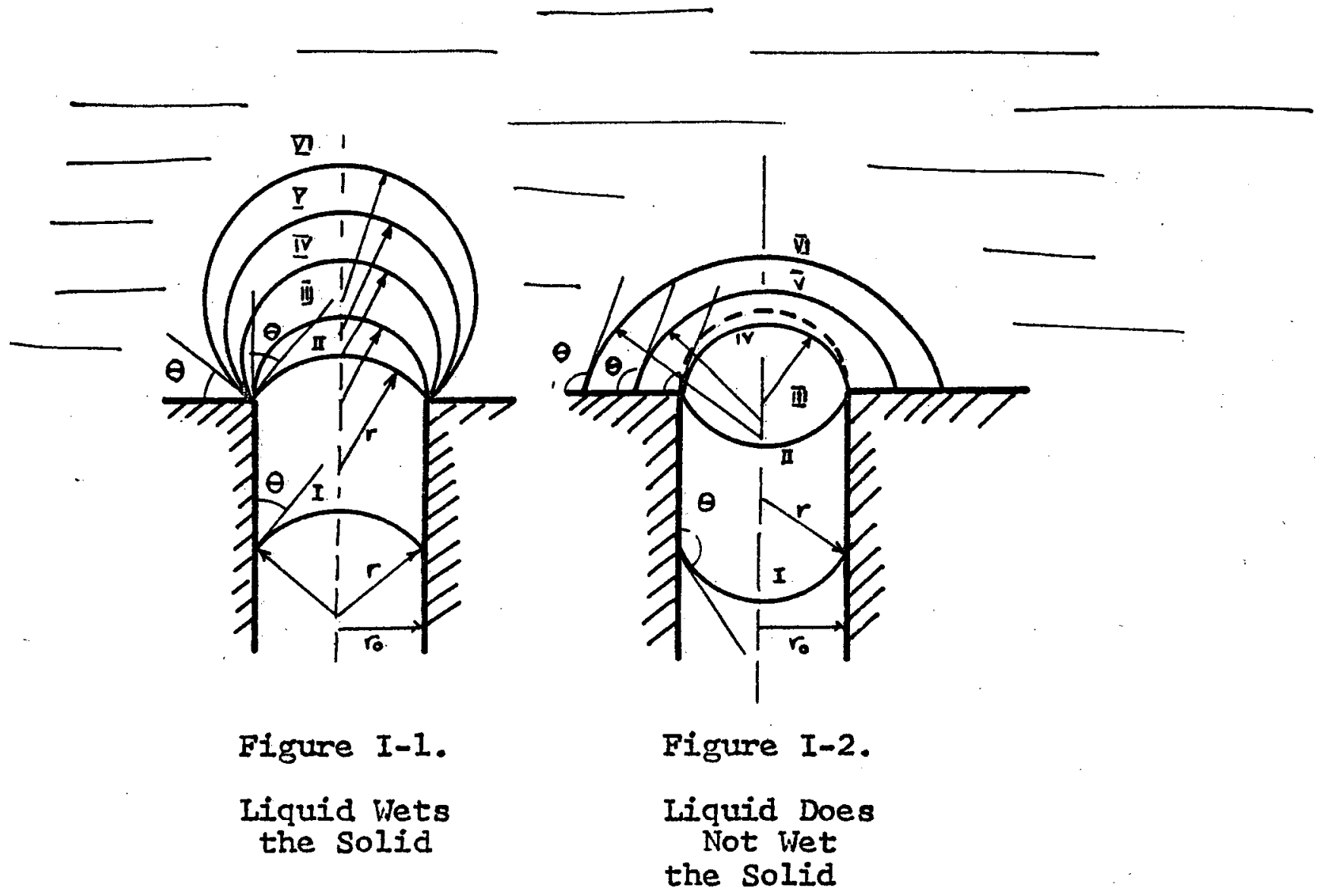

Nucleation from a Cavity

(These figures are reproduced from the paper by Nesis (12).) 
equal then to the radius $r_{0}$, of the cylindrical capillary, thus $r^{*}=r_{0^{*}}$ Consequently, if the cavity is to be active, i.e., if it is to be a nucleating center, the pressure within it must reach the maximum value, corresponding to $r=r^{*}$. Using Clausius-Clapeyron's equation:

$$
\frac{P_{v}\left(r_{0}\right)-P_{s}}{T_{w}\left(r_{0}\right)-T_{s}}=\frac{L}{T\left(v_{v}-v_{s}\right)}
$$

This maximum pressure difference can be related to a temperature djeference. For a cavity which contains only vapor, and for a uniformly superheated liquid, the superheat temperature which will activate a cavity of radius $r_{0}$, is given by Equations $I-I$ and $I-2$, thus

$$
T_{w}\left(r_{0}\right)-T_{s}=\frac{2 \sigma}{r_{0}} \frac{T\left(v_{v}-v_{s}\right)}{L}
$$

It should be noted here that $r_{0}$ is a characteristic of the suriace. Consequently, for a given cavity at a given pressure different starting superheat temperatures can be obtained by varying the value of the surface tension.

If the cavity contains both, vapor and gas, the temperature of the superheated liquid, $T_{\mathrm{w}}\left(r_{0}\right)$, will be reduced by an amount corresponding to the partial 
pressure of the gas.

When the cavity does not vet the solid $(\theta>900)$, the interface is concave (see Figure I-2), and the pressure within the cavity is given by

$$
P=P_{L}-\frac{2 \sigma}{r}
$$

In expanding, the interface changes from concave to convex; the maximum pressure occurs when the radius of the convex interface establishes the contact angle $\theta$, at the entrance of the cavity. Since the radius of curvature, $r$, and the radius of the cavity, $r_{0}$, are related by

$$
r^{*}=\frac{r_{0}}{\sin \theta}
$$

It follows from Equations I-1, I-2 and I-5 that with a non-wetting liquid the cavity will be activated at a lower superheat temperature difference $T_{W}-T_{8}$. Similar deductions can be derived for conical cavities, which were investigated by Courty (13), Bankoff (17), and Wallis (16). Since the radius of curvature of the interface in a conical cavity (see Figure I-3) is given by 


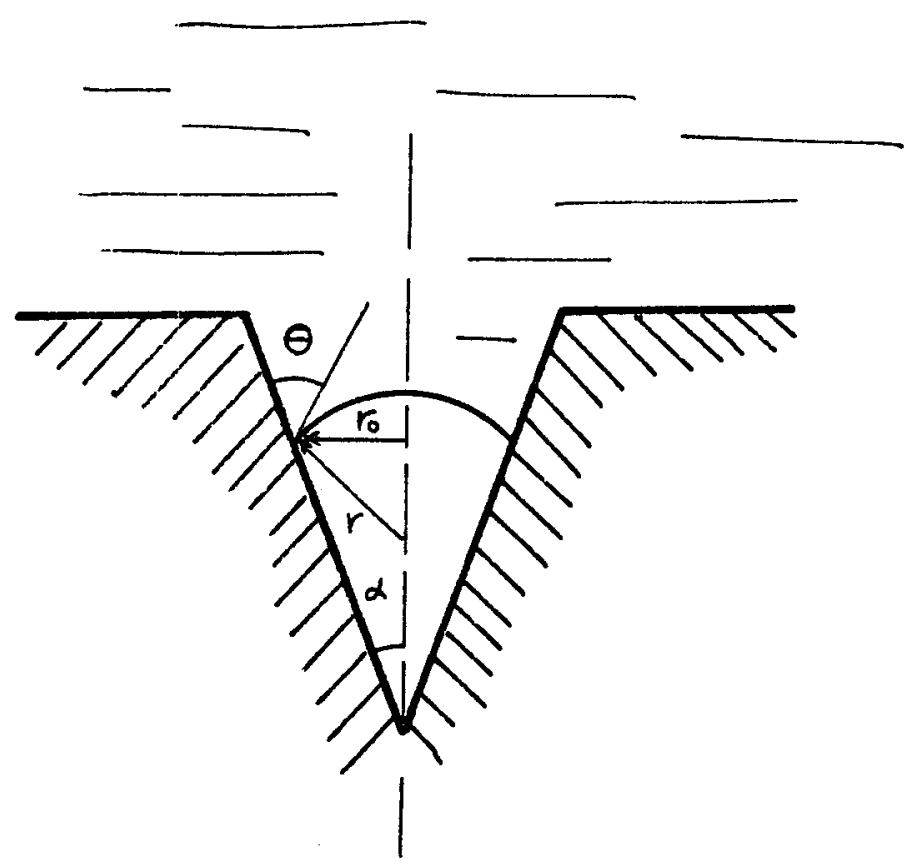

Figure I-3.

Nucleation from a Conical Cavity 


$$
r=\frac{r_{0}}{\cos (\theta-\alpha)}
$$

the maximum superheat temperature will depend on the contact angle $\theta$, as well as on the relative magnitude of $\alpha$ and $\theta$.

The above considerations were verified recently by Griffith and Wallis (16) in experiments which were conducted by reducing the pressure on the system (note that this has the same effect as of uniformly superheating the liquid). The results of these careful and important experiments showed that when $\alpha<\theta<900$ the temperature which activated a cavity of given radius $r_{0}$, was given indeed by Equation $I-3$. However, when the same experiments were performed by heating the surface it was found that the wall superheat temperature, $T_{W}-T_{s}$, required to nucleate a bubble from the same cavity was $20^{\circ} \mathrm{F}$ instead of $3^{\circ} \mathrm{F}$ as predicted by Equation I-3. Similar results were reported by Clark, Strenge and Westwater (10).

Four conclusions can be drawn from these experiments and from the analysis discussed in this section.

1) Pits and cavities which contain entrapped gas or vapor are nucleating centers in boiling 
from a solid surface.

2) The dimensions of the cavity are important in the nucleating process from a heated surface.

3) For a given cavity size a larger superheat is required to nucleate a bubble in a non-uniform than in a uniform temperature field.

4) The nucleating propensity of a surface will depend upon the size distribution of cavities. For a given surface this cavity distribution vill remain an invariant for variations of pressure or of liquid properties. This important fact was shom, also by Griffith and Wallis.

In the nest section we shall discuss experimental results which pertain to bubbles groving and rising from a horizontal heated surface in nucleate, pool boiling, i.e., in absence of forced convection.

I-3. The Dymamics of a Bubble Departing from a Horizontal, Heated Surface

Following the nucleation from a cavity the bubble grows in a superheated liquid film which exists adjacent to the solid wall. The thiclness of this superheated liquid film is of the order of $10^{-1}$ to $10^{-2} \mathrm{~cm} .(2,3$, $4,5,7,20)$. The bubble remains attached to the surface until, at time $t_{d}$, it reaches a characteristic 
diameter $\mathrm{D}_{\mathrm{d}}$, and departs from the heating surface. The departure is governed by the dynamics of the surrounding liquid as well as by the buoyant and adhesion forces. The dynamic effect of the surrounding liquid upon a bubble departing from the surface has not been evaluated quantitatively yet. The problem was analyzed by Wark (21), Fritz (22) and by Nesis (12), who considered a static equilibrium between the buoyant and adhesive forces. For such a static condition the problem becomes an analogue to the problem of determining the maximum volume of a sessile drop hanging from a horizontal surface, a problem which was analyzed by Bashfort and Adams (23). Using their numerical results, Fritz (22) derived the following expression for the diameter $D_{d}$, of a bubble departing from a horizontal surface, thus

$$
D_{d}=0.0208 \theta\left[\frac{\sigma}{g\left(\rho_{L}-\rho_{V}\right)}\right]^{1 / 2}
$$

where the contact angle $\theta$, is measured in degrees. The problem of bubble detachment was investigated experimentally by Jakob and Linle (5) and by Fritz and Ende (24). From a detailed study of motion pictures of steady boiling at low heat flus densities (of the order of $20,000 \mathrm{kcal} / \mathrm{m}^{2} \mathrm{hr}$ ), Jakob and linke (5) found that 
diameters of departing bubbles $D_{d}$, are given by a statistical distribution about a mean value. The values of $D_{\mathrm{d}}$ varied from $1.5 \mathrm{~mm}$ to $4.5 \mathrm{~mm}$ with a maxcimum at about $\mathrm{D}_{\mathrm{d}}=2.8 \times 10^{-1} \mathrm{~cm}$. This marimum value was in agreement with the value predicted by Equation I-7 for a contact angle of $\theta=50^{\circ}$. It was observed also that preceding the detachment, bubbles become elongated (see Figure 1-4). Immediately after the detachment, i.e., after the elongated connection snaps, the lower surface of the bubble reenters causing a deformation. Thus, imnediately after departure, the bubble tales first a mushroom-like form and later a lenticular shape.

It was observed in these experiments that the nucleation and bubble growth from a given nucleating center is a periodic process. Following the departure of a bubble the colder liquid comes in contact with the solid and gets heated during a "contact time", $t_{c}$, at the end of which time another bubble is nucleated from the same center. This new bubble grows until, at time $t_{d}$, it in tum departs from the surface, and the process is repeated. The contact time, $t_{c}$, during which the liquid in the vicinity of the nucleating cavity becomes superheated was found to be approsimately equal to the growth time $t_{d}$. Thus, the total frequency of bubble emission 


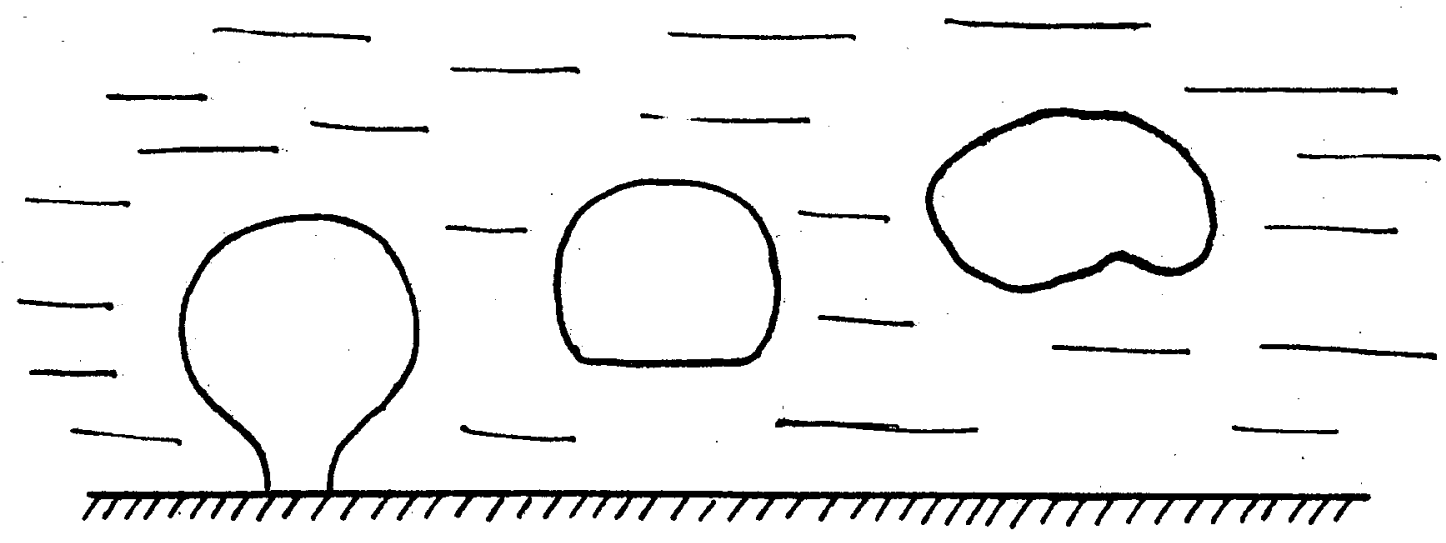

Figure I-4.

Deformation of a Bubble Departing from

a Heated Horizontal Surface According to Jakob (3). 


$$
f=\frac{1}{t_{c}+t_{d}}
$$

was found to be a constant for a given nucleating center, although for different cavities a statistical distribution was observed. In the range of low heat flux densities, i.e., in the range which was investigated by Jakob and linke and Fritz and Ende it was found that both the diameter $D_{\mathrm{d}}$, and the frequency $f$, were independent of the heat flux. The effect of increasing the heat flux was to increase only the number of active nucleating centers. Consequently the product of the diameter $D_{d}$, and the frequency $f$, is a constant,

$$
D_{d} \cdot F \Rightarrow \text { const. }
$$

Recently a study of bubble dynamics in the entire range of nucleate boiling with methanol was reported by Perkins and Westwater (25). It was observed that the average bubble diameter as well as the average frequency remained constant for heat fluxes up to $80 \%$ of the critical heat flux. At higher flux densities both the diameter as well as the frequency increased. In these 
experiments at high heat transfer rates the contact time, $t_{c}$, was found to be approximately zero, i.e., the bubbles form immediately after each other. A similar observation was reported by Donald and Haslam (26). This change of bubble generation from intermittent to continuous was investigated by Yamagata and Nishikawa (27). These experiments were carried out with water at atmospheric pressure with heat flux densities ranging from 6,000 to $13,000 \mathrm{kcal} / \mathrm{m}^{2} \mathrm{hr}$. It should be noted here that for water at atmos pheric pressure the critical heat flux is approximately $10^{6} \mathrm{kcal} / \mathrm{m}^{2} \mathrm{hr}$. From a study of their esperimental data, Yamagata and Nishikawa classified bubbles into four groups, i.e., sphere type, bell type, precession type and tandem type. Figure I-5, which is reproduced from their report, shows the different bubble forms.

The formation of sphere and bell type bubbles was essentially identical, consequently they were classified as "simple bubbles"; whereas the precession and the tandem bubbles vere called "multiple bubbles" because they were formed by bubble coalescence. All four bubble types were generated intermittently. A contact time, $t_{c}$, and a growth tine, $t_{d}$, were observed for simple bubbles. The product of $D_{d} . f$ was constant for simple bubbles, whereas the data for nultiple bubbles showed a 


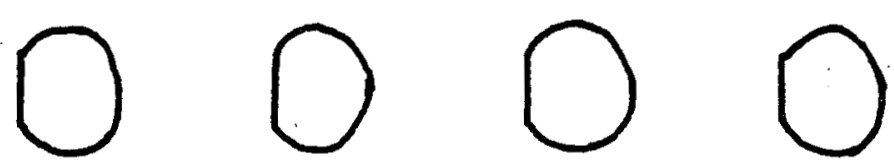

a) Sphere Type
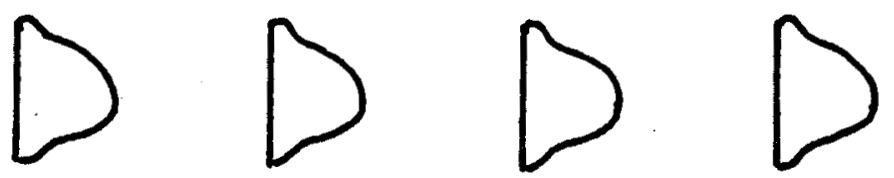

b) Be11 Type

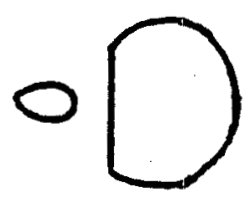<smiles>O=C1CCCCCCC1</smiles>

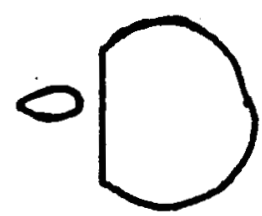

c) Precession Type<smiles>C1CCCCCC1</smiles><smiles>C1CCCCCCC1</smiles><smiles>C1CCCCC1</smiles><smiles>C1CCCCCCCCCC1</smiles>

d) Tandem Type

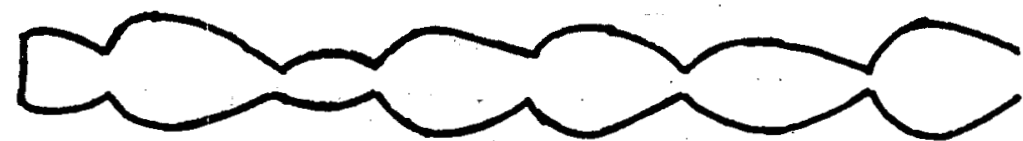

e) Continuous Vapor Column

Figure I-5. Types of Vapor Bubbles Departing from a Horizontal Heated Surface as Reported by Yamagata and Nishikawa (Reference 27). 
larger scatter. Simple bubbles were generated at lower heat flux densities when a smaller number of nucleating centers were active. Multiple bubbles were formed at higher heat flux density when the bubble columns were more numerous. Yamagata and Nishikawa noted that when the heat flux is increased beyond a certain limit "the bubbles were not generated intermittently, but formed a continuous vapor column. These whirls were often generated at two or more spots interferring with each other." Such an observed continuous vapor column is shown in Figure I-5. It is important to note that continuous vapor columns can be seen also in the high speed movies taken by Perkins and Westwater.

We shall now summarize the experimental evidence concerning the dynamics of bubbles departing from a horizontal surface in nucleate, pool bolling.

1) At low heat flux densities the mechanism governing the departure can be approximated by a quasi-static equilibrium between the buoyant and adhesive forces. Consequently, the statistical mean value of the diameters can be approximated by Equation I-7.

2) Immediately after the departure a bubble undergoes a deformation. 
3) At low heat transfer rates bubbles are generated intermittently with a characteristic constant frequency.

4) As the heat flux is increase the contact time, $t_{c}$, decreases. Bubbles change from simple to multiple type which are formed by a coalescence of several bubbles.

5) At high heat transfer rates the contact time decreases until bubbles form immediately after each other.

6) At still higher heat transfer rates the diameters and the frequency of bubble formation increase until a continuous vapor colum is formed.

7) It appears that at low heat transfer rates the effect of increasing the heat flus is to increase the number of active nucleating centers, ie., of bubble columns. Whereas at higher heat flux density this effect appears to change the bubble generation from an intermittent to a continuous process. The characteristic of the phenomenon thus appears to be a change from a column consisting of single, rising bubbles to a continuous vapor column, i.e., a vapor jet. 
I-4. The Relation Between Heat Flux Density, Surface Roughness and Temperature in Nucleate Boiling

In the two previous sections we have reviewed the nucleation of a single bubble from a cavity and the departure of a bubble from a heated surface. In this section we shall discuss the interdependence of the heat flux density, surface roughness, superheat temperature difference and bubble dynamics in nucleate boiling. The phenomenon will be described in more detail in order thet, In Chapter III, it may be more easily compared to the process of a gas bubbling through a porous plate. The discussion in this section is based to a large extent upon a penetrating study of nucleate boiling which was reported by Courty (13) and Courty and Foust (14). Consider a surface with cavities which contain some entrapped gas or vapor and let the heat flux density increase so that the temperature of the solid surface exceeds the saturation temperature, i.e., the liquid adjacent to the wall becomes superheated. At some superheat temperature difference, $T_{w}-T_{8}$, a few bubbles will start growing from some nucleating centers located at random. At what particular superheat temperature this nucleation can start will depend, as discussed in Section I-2, upon the dimensions of the cavities, contact angle and the amount of entrapped 
gas or vapor. Consequently, surfaces with different surface characteristics will have different "starting" superheat temperatures. The heat flux starts deviating from the values corresponding to natural convection when few of these active bubble centers appear. If the heat flux density is increased again, the superheat temperature increases (though at a lower rate than in natural convection), and the active bubbling sites become centers of patches of more violent boiling. Courty and Foust observed that, in nucleate, patchwise boiling, wide variations occur in the temperature of the surface. The areas covered with bubbles are considerably cooler than the bubble-free areas. Also, widely varying temperature readings were recorded by thermocouples located in the surface. The magnitude of these fluctuations were, however, not reported. Since the original bubbling centers are located at random, the patches of active boiling are also randomly distributed.

Courty and Foust discussed two processes by which patchwise boiling can be established around an active nucleating center. The first is a pressure fluctuation mechanism, and the second is a vapor trapping process. The pressure fluctuation mechanism bears similarity to cavitation. During the bubble growth the liquid is pushed away from the nucleating center; after the 
departure of the bubble, the liquid rushes back. These oscillations of the fluid and the possible vortex formation with attendant pressure variations can induce nucleation.

The second mechanism is a vapor-trapping process. Suppose that in the vicinity of the active center are cavities which are, originally, filled with liquid. The bubble growing from the active nucleus may blanket some of these neighboring cavities. After the bubble breaks off, the liquid advances over the area which was covered by vapor and may trap some vapor in the neighboring capillaries. If the heating rate is sufficiently high and the rate of penetration of the advancing liquid comparatively slow, the liquid may become superheated before it refills the capillary. Under these conditions the neighboring capillaries may in turn become active nucleating centers. It is seen that this process depends upon the dimensions of the capillaries as well as upon two rates, the rate of heating the liquid and the rate of penetration of the liquid into cavities. This problem was malyzed recently by Banlooff (18).

The process of seeding, i.e., of activating cavities around the original active nucleating center, will continue until, depending upon the heating rate, the original nucleus can co-exist independently with 
another nucleus. The internediately located nucleating centers will be starved out. Such starving of a nucleus by a new active cavity was described recently by Clark, Strenge and Westwater (10). At a given heat flus density the spreading of boiling patches will cease when there are no cavities, within the range of activation, that can support a new bubble colum at the prevailing heating rate and superheat temperature, $T_{W}-T_{S}$. If the heat flux density is increased again, the temperature of the non-bubbling, ie., the bare area which surrounds the boiling patch, increases. Consequently, within the range of activation new, smaller cavities (which require higher superheats) can be activated and at the higher heating rates additional bubble columns can be maintained. This spreading mechanism continues with increasing heat flus densities until the whole area is popilated with nucleating centers, i.e., with bubble columns.

Two important facts should be stressed now. It was observed already tiiat the superheat temperature difference, $T_{\mathrm{w}}-T_{S}$, at which bubbling starts depends upon the conditions of the surface. Different "starting" superheats are needed for different cavities. It was noted also that when boiling occurs in patches, the increase of superheat temperature which follows an 
increase of the heat flux density depends on the size distribution of cavities. Different superheat temperatures are needed to activate new nucleating centers. Thus in nucleate boiling, at moderate heating rates at least, the rate of increase of the superheat temperature $T_{w}-T_{s}$, with an increase of the heat flux density depends upon the characteristics of the surface. This is shown on Figure I-6 which is reproduced from the paper by Courty and Foust. It is seen that different "starting" superheat temperatures, different nucleate boiling curves with different slopes can be obtained with the sarne metal surface having different roughness characteristics. Thus, the heat flux density is not a single valued function of the temperature. In order to determine the relation between the heat flus and the temperature, the characteristics of the surface must be specified.

The question which arises - whether this depe ndence upon the surface roughness is a characteristic of the entire nucleate boiling curve - is not resolved yet. Experimental results of Jaloob ( 3 ) and of Averin (28) iridicate that, for water at atmos pheric pressure, nucleate boiling curves for surfaces of different roughness merge when the heat flux density reaches a value of 150,000 to $200,000 \mathrm{kcal} / \mathrm{m}^{2} \mathrm{hr}$. Note that the 


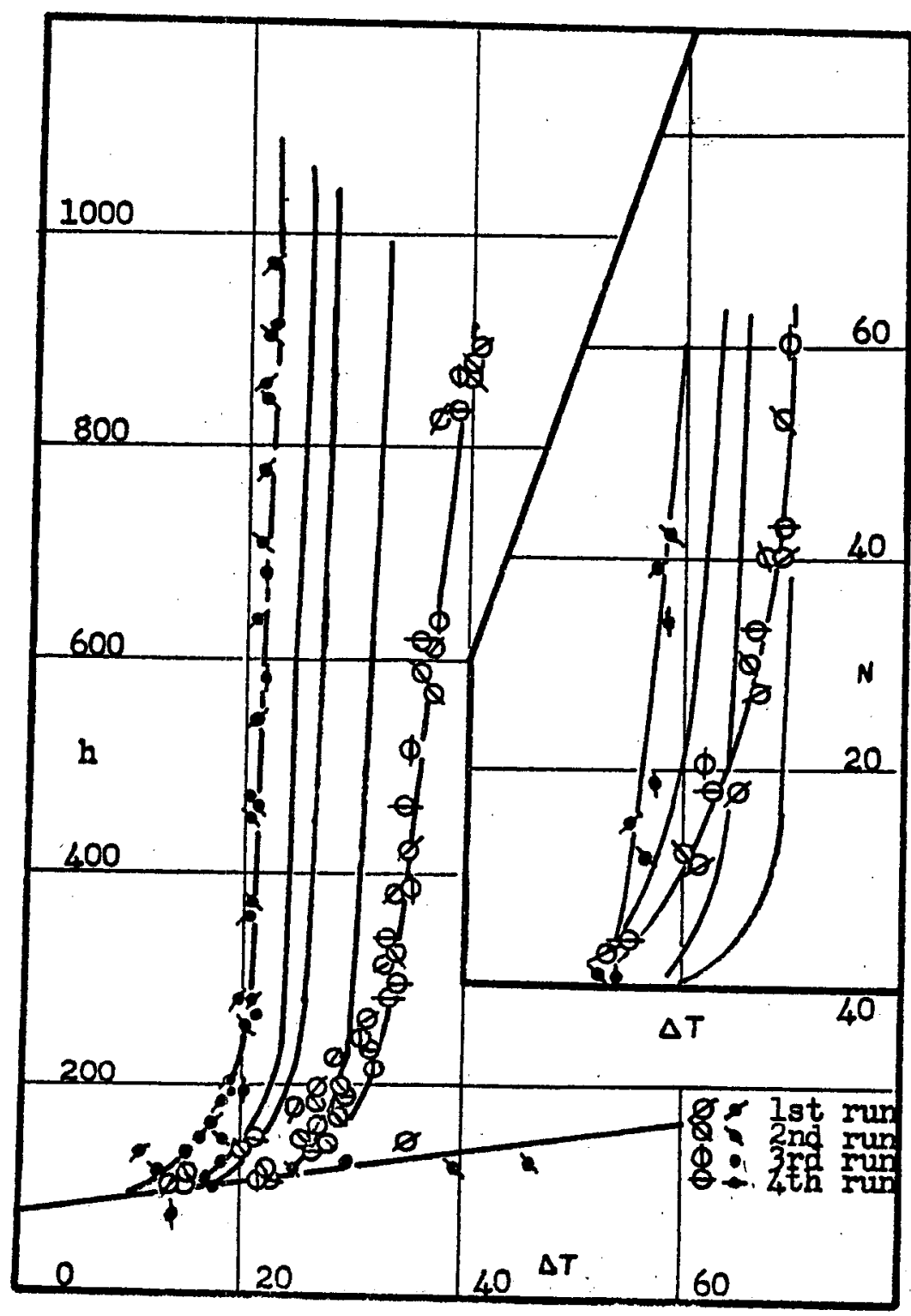

FIGURE I-6. THE EFFECT OF DIFFERENT AMOUNTS OF ROUGHINESS ON THE HEAT TRANSFER IN NOCLEATE BOILING ( $h=q / \triangle T, B T U / \mathrm{HR}$ FT2OF; $N=$ BUBBLES/IN2). THIS FIGURE IS REPRODUCED FROM THE PAPER BT CLAUDE CORTY AND ALLAN S. FOUST $(13,14)$. 
critical heat flux is $10^{6} \mathrm{kcal} / \mathrm{m}^{2} \mathrm{hr}$. The dependence of the nucleate boiling curve upon the surface roughness can be expected to manifest itself in the low flux region because boiling in patches occirs at lower heat flus densities. The merging of the nucleate boiling curves could be interpreted then as a change in the boiling process, i.e., the termination of boiling in patches. A large number of active nucleating centers would tend to decrease the effects of different surface roughness. It was discussed in the preceding section that with increasing heat transfer rates bubbles change from simple to multiple, and the bubble generation changes from an intermittent to a continuous process. It is possible that with the spreading and the subsequent disappearance of boiling patches a gradual change in the bubble types and in the bubble generation mechanism takes place over most of the surface area. Such a change, together with the termination of boiling in patches, could account for the change in the boiling process. It appears also that the effect of the surface roughness disappears for surfaces which have "aged", i.e., from which boiling was maintained over a period of a week or so. The experiments of Jakob and Linke (4) and of zysina-lolozhen (29) indicated that both the superheat temperature difference, $T_{W}-T_{6}$, and the 
number of nucleating centers, $n$, approach a limit value with prolonged boiling. These limiting values were Independent of the original roughness, but dependent upon the metal of the heating surface. Zysina-Molozhen noted that the number of nucleating centers and, therefore, the superheat temperature, become constant (For a given surface) once a stable oxide film has formed. She made the following observation: "Evidently the factor controlling the numerical value of the heat transfer coefficient in ordinary heating surfaces is the thermal conductivity of the oxide film, which males it impossible to determine the true value of the heat transfer coefficient." Note that her statement implies the uncertainty of determining the superheat temperature $T_{w}-T_{8}$, for a given heat flux density. We shall return, in Chapter III, to discuss this effect in connection with the coupling of the themal properties of the solid and liquid during the transient temperature oscillations which occur in boiling. In the following we shall summarize the discussion of this section.

1) The superheat temperature difference, $T_{w}-T_{B}$, at wich nucleate boiling sets in depends upon the characteristics of the surface and upon the amount of gas and vapor which is entrapped in cavities. The first nucleating centers are 
located at randorn.

2) At low heat transfer rates boiling takes place in patches which are located around the first nucleating centers.

3) During this non-uniform, patchwise bolling spatial and time variations occur in the temperature of the surface.

4) In the boiling patches, growing and departing bubbles strongly agitate the superheated film; colder fluid is brought in contact with the heating surface resulting in high heat transfer rates.

5) With an increase of the heat flux density, the superheat temperature, $T_{W}-T_{8}$, increases so as to alter the number of nucleating sites. The increase of active cavities and, therefore, of the superheat, is such that the agitation of the superheated film due to all bubble columns can accomnodate the new heat flux.

6) In nucleate boiling, at moderate heat transfer rates at least, the heat flux is not a singlevalued function of the temperature. In order to determine the relation between the heat transfer rate and the temperature of the surface, the characteristics of the surface 
must be specified.

7) It appears that, at higher heat flux densities, the effect of the surface roughness upon the q $-\Delta T$ relation vanishes, indicating a more uniform boiling (disappearance of patches) with a possible change in the types of bubbles and in the bubble generation process (from intermittent to continuous).

8) It appears also that the effect of the surface roughness on nucleate boiling disappears once a stable oxide film coats the surface. Under these circumstances it appears that the thermal conductivity of the oxide film becomes the controlling factor.

In this part of the dissertation we have discussed the results of analytical and experimental investigations which pertain to the nucleation process, to the hydrodynamics of bubbles departing from surface and to the effects of surface roughress. We shall proceed, in Chapter II, with the problem of bubble growth. 
CHAPTER II

THE PROBLEM OF BUBBLE GROWTH

II-1. The Dynamic s of Vapor Bubbles in a Uniform

Temperature Field - Historical Bacliground

The growth of a bubble in a superheated liquid

was first analyzed by Bosnjakovic (30). According to

his theory the vaporization process is maintained by an energy transfer from the superheated liquid to the bubble interface. The temperature drop which maintains this process is localized in a thin boundary layer which surrounds the bubble. This model is shown on Figure II-1. A relation between the bubble growth rate, the temperature drop and the heat transfer coefficients is obtained from an energy balance, thus

$$
h\left(T_{0}-T_{s}\right)=L P_{y} \frac{d R}{d t}
$$

The theory of Bosnjalkovic was tested experimentally by Jakob and co-workers $(2,3,4,5)$, Prüger (31) and Fritz and Ende (24); good agreement was reported. In the discussion of their experimental results Fritz and Ende noted that, for a liquid which is initially uniformly 


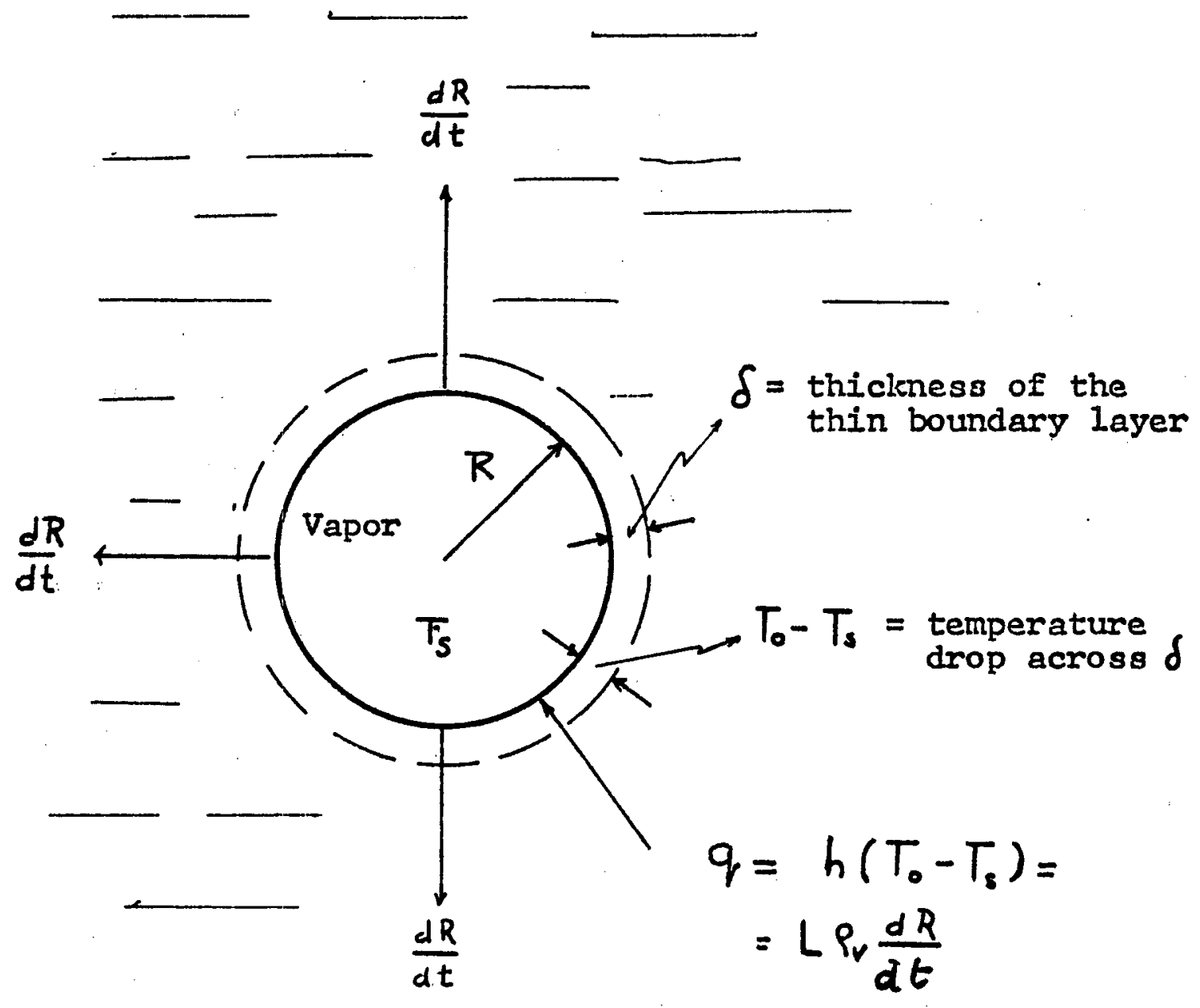

Liquid at temperature: $T_{0}$

Figure II-1. Bosnajalcovic's Analytical Model of a Bubble Growing in a Uniformly Superheated Liquid 
superheated, the bubble growth problem was similar to the one dimensional, transient heat conduction problem, 1.e., to

$$
\begin{gathered}
a \frac{\partial^{2} T}{\partial x^{2}}=\frac{\partial T}{\partial t} \\
t=0: T(x, 0)=T_{0}-T_{S} \\
t>0: T(0, t)=0
\end{gathered}
$$

The heat transfer coefficient for a growing bubble can be obtained from the gradient at the bubble wall $(x=0)$, thus

$$
h\left(T_{0}-T_{s}\right)=\kappa\left(\frac{\partial T}{\partial x}\right)_{x=0}
$$

The temperature gradient is obtained by solving Equation II-2, thus

$$
\left(\frac{\partial T}{\partial x}\right)_{x=0}=\frac{T_{0}-T_{5}}{\sqrt{\pi a t}}
$$

Substituting Equation II-3 and II-4 into Equation II-I, the bubble growth rate is given by

$$
L P_{r} \frac{d R}{d t}=K \frac{T_{0}-T_{s}}{\sqrt{\pi a t}}
$$

Using high speed movies Fritz and Inde determined the bubble growth velocity, and from Equation II-I computed the heat transfer coefficient. This value was then 
compared to the value computed from Equations II-1 and II-4; the two values of $h$, thus determined, were found to be in good agreement with each other.

At first this agreement could appear as fortuitous. The real bubble is spherical; its interface represents a moving boundary whereas the system described by Equation II-2 represents a semi-infinite slab with stationary boundary. Also, the effects of Iiquid inertia and of surface tension. were neglected in the analysis. Nevertheless, subsequent more detailed studies of the bubble growth problem only corroborated the theory of Bosnjakovic and the analysis of Jakob, Fritz and Ende which contains the essential feature of the phenomenon. The first mathematical formulation which took into account the effects of liquid inertia, surface tension, sphericity and of the moving boundary is due to Plesset and Zwick $(32,33)$ and to Romie (34). The problem is formulated by considering Rayleigh's equation of motion:

$$
R \ddot{R}+\frac{3}{2} \ddot{R}^{2}+\frac{2 \sigma}{\rho_{L} R}=\frac{P_{L}(t)-P_{s}}{\rho_{L}}
$$


Clausius-Clapeyron's equation:

$$
\frac{P_{r}(t)-P_{s}}{T_{r}(t)-T_{s}}=\frac{L \rho_{v} \rho_{L}}{T\left(\rho_{L}-\rho_{v}\right)}
$$

and the energy equation:

$$
\begin{gathered}
a\left[\frac{\partial^{2} T}{\partial r^{2}}+\frac{2}{r} \frac{\partial T}{\partial r}\right]=\frac{\partial T}{\partial t}+\frac{\partial T}{\partial r} \dot{r} \\
t=0: T(r, 0)=T_{0}-T_{s} \\
t>0: \quad r\left(\frac{\partial T}{\partial r}\right)_{r}=R(t)=L P_{V} \dot{R} \\
T(\infty, t)=T_{0}-T_{s}
\end{gathered}
$$

The problem was analyzed in References 32,33 and in 34, 35. The results of these studies were in agreement with each other and with experimental data reported by Dergarabedian (36). It was found that during the growth of a bubble the effect of liquid inertia and of surface tension are not important. Consequently, the growth of a vapor bubble is governed by the heat transfer process. Plesset and Zwick derived the following expression for the bubble growth rate

$$
L e_{v} \stackrel{R}{=}=\sqrt{3} \times \frac{T_{0}-T_{s}}{\sqrt{\pi a t}}
$$


Whereas Forster and Zuber obtained

$$
L P_{v} \stackrel{\mathrm{R}}{=} \frac{\pi}{2} \times \frac{T_{0}-T_{s}}{\sqrt{\pi a t}}
$$

It should be noted that, in both of these studies, the assumption was made that the temperature drop is localized in a "thin boundary layer" near the bubble wall. Thus, the "thermal boundary layer" assumption of Bos njakovic was reintroduced in the problem.

Recently, Birlkhoff, Margulies and Horning (37) and Scriven (38) solved the energy problem (Equation II-8) without maleing recourse to the "thin boundary layer" approsimation. It was shown that this assumption is valid when the Jakob number* is large, that is when:

$$
J_{a} \triangleq \frac{\left(T_{0}-T_{S}\right) C P_{L}}{L P_{V}} \gg 1
$$

It was found also, that for large Jakob nurabers the bubble growth rate is given by Equation II-9.

* This dimensionless group was recently proposed by Savic (39) to be referred to as the "Jakob Number" in honor of the late Professor Max Jakob. 
Comparing Equation II-9 and II-10 with Equation II-5 it is seen that, apart from a numerical constant, these equations are identical. A larger growth rate could have been expected for spherical bubbles than for a plane interface because the temperature gradient at a spherical surface is given by (40)

$$
\left(\frac{\partial T}{\partial r}\right)_{r=R}=\left(T_{0}-T_{s}\right)\left[\frac{1}{\sqrt{\pi a t}}+\frac{1}{R}\right]
$$

It follows for Equation II-4 and II-12 that the plane approsimation is valid when

$$
\frac{\sqrt{T a t}}{R} \ll 1
$$

It is important to note that, for growing vapor bubbles, Equation II-9 and Equation II-10 indicate that the effect of sphericity is only to increase the gradient by a constant factor whose value lies between 1 and $\sqrt{3}$, with $\pi / 2$ an intermediate value.

We can conclude this section by noting that for a growing vapor bubble the effects of Iiquid inertia and surface tension are not important. The growth rate, i.e., the rate of evaporation is given by an equation of the form: 


$$
L P_{v} \dot{R}=b \leqslant \frac{T_{0}-T_{s}}{\sqrt{\pi a t}}
$$

Where the value of the constant is $b=1$ for "plane" bubbles, or $b=\pi / 2$ or $\sqrt{3}$ when correcting for the curvature. The mathematical formulation originally given by Fritz and Ende, and the theory of Bosnjakovic and Jakob contain, therefore, the essential feature of the phenomenon. In the next section we shall extend the theory of Bosnjakovic and Jalkob to include the effect of a non-uniform temperature field.

\section{II-2. The Growth of a Vapor Bubble in a Non-Uniform}

Temperature Field

In 1932 Jakob (3) described the heat transfer process which occurs at the interface of a bubble groving on a heated surface as follows: "It can be imagined that during the small explosion which starts the growth of a bubble, the interface temperature, because of the heat of vaporization, drops immediately from the superheat temperature to the saturation temperature, for example from $110^{\circ} \mathrm{C}$ to $100^{\circ} \mathrm{C}$. . As a consequence of the heat transfer from the liquid to the vapor bubble the liquid envelope is being cooled progressively from the inside toward the outer boundary; a temperature 
boundary layer is created with a constantly decreasing temperature drop. This thermal boundary increases in thickness until the thermal wave, which advances from the vapor bubble interface into the liquid, has reached the outer limit of the hydrodynamic boundary layer. The decrease in thickness of the hydrodynamic boundary layer because of the evaporation at the interface is, initially, a small fraction of the total thickness." This model is shown on Figure II-2.

It is important to point out now the difference between the growth of a bubble in a uniformly superheated liquid and the growth in a non-uniform temperature field. In a uniformly superheated liquid (see Figure II-1) the thickness of the thermal boundary layer $\delta$, constantly increases; however, since the liquid was initially at a uniform temperature $T_{0}$, the total temperature difference $T_{0}-T_{s}$, across the thermal boundary remains constant. In a non-uniform temperature field (see Figure II-2) the thicleness of the thermal boundary layer $\delta$, also increases as the thermal waves advance into the liquid. However, in this case, since the initial temperature in the liquid was not uniform, the temperature drop across the thernal boundary layer $\delta$, constantly decreases as $\delta$ increases. In other words, whereas in a uniform thernal field only one heat transfer process occurs, 


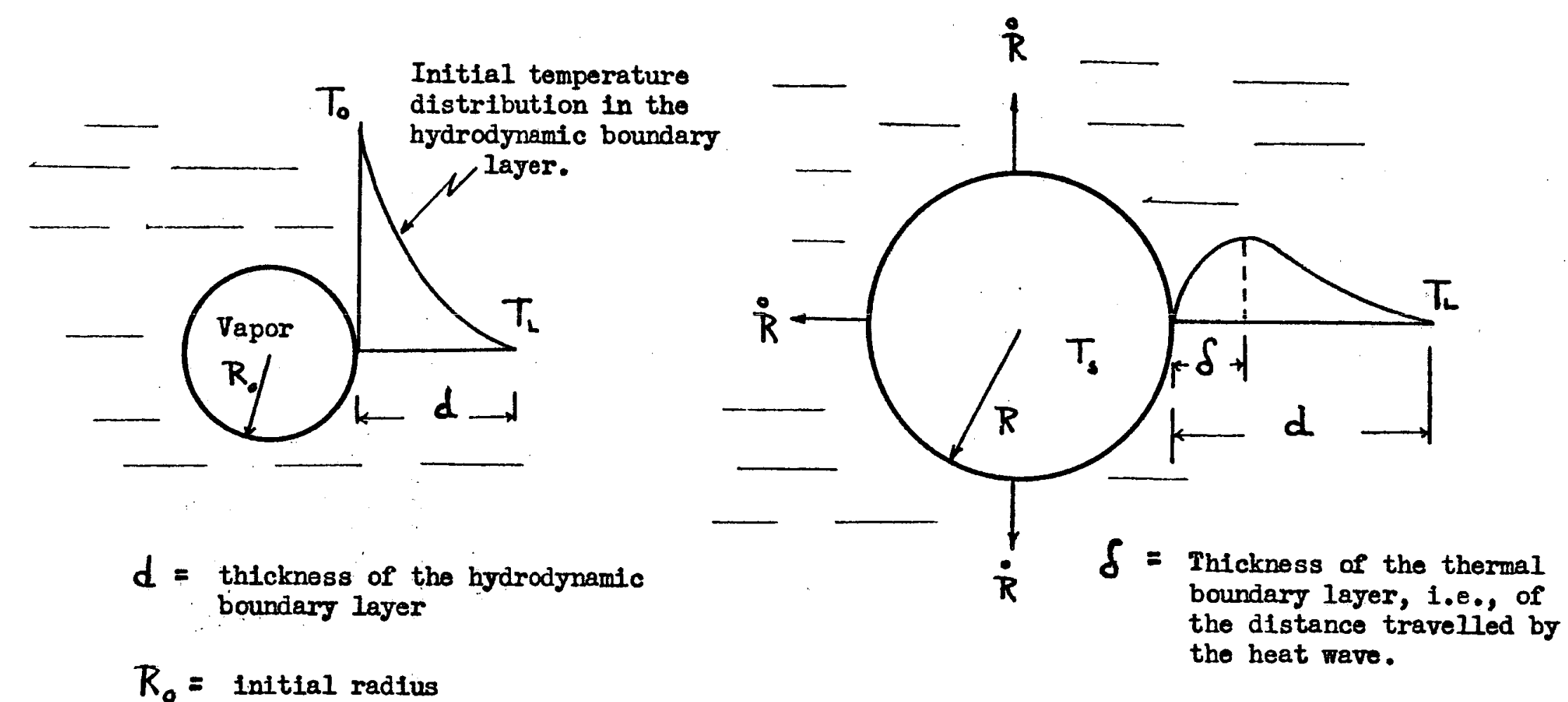

FIGURE II-2.a. INITIAL TEMIPERATURE DISTRIBUTION

FIGURE II-2.b. TEMPERATURE DISTRIBUTION FOR A GROWING BUBBLE

FIGURE II-2. JAKOB'S ANALYTICAL MODEL OF A BUBBLE GROWIKG IN A NON-UNIFORM TEMPERATURE FTEID 
In a non-uniform temperature field two transfers of energy take place. One is the heat transfer across the film $\delta$; this process maintains the evaporation at the bubble interface. The second is the heat transfer to the bulk liquid.

The theory of Bosnjakovic and Jakob can be extended to include the effect of the non-uniform temperature by maling an energy balance per unit transfer area. For a plane bubble the rate of evaporation is then given by:

$$
L p_{r} \dot{R}=k \frac{T_{0}-T_{b}}{\sqrt{\pi a}}-q_{b}
$$

where the second term on the right hand side is the heat transfer rate to the bulk liquid. For a uniformly superheated liquid $q_{b}=0$ and Equation II-15 becomes Equation II-5. In view of the results which were discussed in the previous section it can be expected that a correction for the curvature can be made by multiplying the right hand side of Equation II-15 by $b=\pi / 2$, thus

$$
L P_{v} \stackrel{\circ}{R}=b\left[K \frac{T_{0}-T_{s}}{\sqrt{\pi a t}}-q_{b}\right]
$$


with $b=1$ or $b=\pi / 2$. The bubble radius is then given by

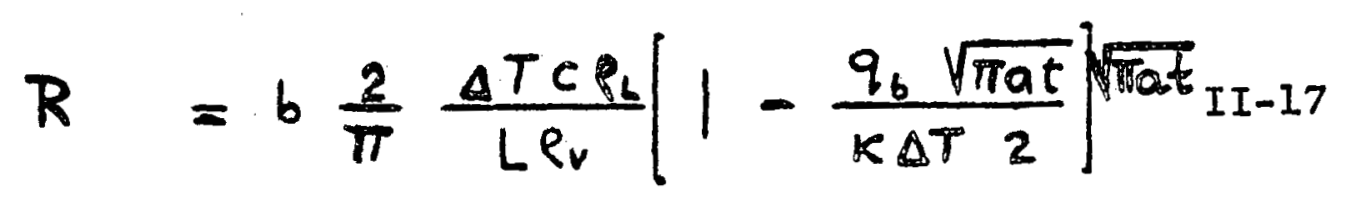

In order to make use of Equation II-I7 we must determine the heat flux $\mathrm{q}_{\mathrm{b}}$, to the bulk liquid. Consider an instant just prior to the nucleation of a bubble. A temperature gradient exists in the liquid adjacent to the heating surface. It is this gradient which transfers the energy from the solid wall to the liquid. Consider now the instant just after the nucleation of a plane bubble. The effect of introducing, locally, the vapor phase between the solid and the liquid is to insulate, locally, the liquid from the heating solid. Assuming that no great distortions and disturbances occur in the liquid during this transition instant, the temperature gradient between the vapor interface and the liquid is approximately equal to the gradient which existed between the solid wall and the liquid before the bubble wes nucleated. In the sections which follow we shall identify therefore, $q_{b}$ with the heat transfer rate from the heating surface, $q$ - This appears a rather drastic assumption; we shall justify 
it a posteriori in the sections which follow after we esamine the physical process in greater detail.

It is seen that Equation II-17 relates the bubble radius to the superheat temperature $\left(\Delta T=T_{W}-T_{8}\right)$ and the heat flur which exist locally around a nucleating center. It was discussed in Chapter I that, in nucleate boiling at low heat flux density, both the temperature of the surface and the heat transfer rate vary over the heated area. The values which are usually reported are average values. It is known also from the experiments of Jakob and Iinke (5), of Ellion (15) and of Treschov (41) that the diameters of bubbles departing from or collapsing on a heated surface follow a distribution curve. Therefore, if we introduce into Equation II-17 the average heat flux and superheat we can expect to predict only the growth of an average bubble and not of a specific bubble (unless it coincides with the average one) whose grorth was recorded on a motion picture. Keeping this fact in mind we shall examine first the growth of a bubble in a liquid whose bulk is at saturation temperature. 
II-3. The Bubble Growth in Liquids at Saturation Temperature

In this section the bubble growth rate predicted by Equation II-17 will be compared to experimental results reported by Fritz and Ende (24) and by Zmola (42). In Section I-3 the experiments of Jalcob and Linke (5) were discussed; in these experiments the diameters of bubbles departing from a heated surface varied between $1.5 \mathrm{~mm}$ and $4.5 \mathrm{~mm}$ with a maximum at about $\mathrm{D}_{\mathrm{d}}=2.81 \times 10^{-1} \mathrm{~cm}$. Because Jakob and Iinke did not report the values of the superheat temperature $T_{W}-T_{s^{\prime}}$ and the heat flux density $q$, Equation II-17 cannot be used for comparison with their results. This can be done, however, with the data for water reported by Fritz and Ende which is tabulated below.

\section{TABLE II-1}

Experimental Data of Fritz and Ende

$\begin{array}{llcccc}\begin{array}{l}\text { Bubble } \\ \text { Number }\end{array} & \frac{\mathrm{Kcal}}{\mathrm{hrm}^{2}} & \begin{array}{c}\mathrm{T}_{\mathrm{w}}-\mathrm{T}_{\mathrm{s}} \\ { }^{\circ} \mathrm{C}\end{array} & \begin{array}{c}\mathrm{t}_{\mathrm{c}} \\ \mathrm{sec}\end{array} & \begin{array}{c}\mathrm{t}_{\mathrm{d}} \\ \mathrm{sec}\end{array} & \begin{array}{c}\mathrm{D}_{\mathrm{d}} \\ \mathrm{cm}\end{array} \\ \mathrm{BMI} & 19000 & 9 & 0.0225 & 0.023 & 5.42 \times 10^{-1} \\ \mathrm{BM3} & 19000 & 9 & 0.0025 & 0.018 & 4.04 \times 10^{-1} \\ \text { BR8 } & 19000 & 9 & 0.01675 & 0.020 & 4.64 \times 10^{-1}\end{array}$


Inserting the above values of superheat temperature $T_{w}-T_{s}$, heat flux $q$, and time at departure $t_{d}$, into Equation II-17 the corresponding diameters at departure for two values of the constant $b$ are given below.

TABLE II-2

Diameters at Departure Predicted by Equation II-I7

$\begin{array}{ccr}\begin{array}{c}\text { Bubble } \\ \text { Number }\end{array} & D_{\mathrm{d}}(\mathrm{cm}) & D_{\mathrm{d}}(\mathrm{cm}) \\ \mathrm{b}=1 & \mathrm{~b}=\frac{\pi}{2} \\ \text { BMI } & 2.91 \times 10^{-1} & 4.58 \times 10^{-1} \\ \text { BM3 } & 2.68 \times 10^{-1} & 4.18 \times 10^{-1} \\ \text { BR8 } & 2.76 \times 10^{-1} & 4.33 \times 10^{-1}\end{array}$

It is seen that the predicted values are in better agreement with experiments when the correction for the sphericity is taken into account. This could have been expected because for large bubbles the liquid laminae over the bubble are stretched thus increasing the temperature gradients. We note, however, that even if this correction was not included, the predicted values for the "plane" bubble, i.e., $b=1$, are in agreement with the average value observed by Jakob and Linlee, i.e., $D_{\mathrm{d}}=2.81 \times 10^{1} \mathrm{~cm}$. 
In order to investigate whether Equation II-17 Is in agreement with experimental data over a longer time interval we can compare it to the experimental data reported by zmola (42) and shom on Figure II-3. For these experiments the heat flux was $q=9.6 \times 10^{3}$ $\mathrm{Btu} / \mathrm{hr} \mathrm{ft}^{2}$, and the superheat temperature $\mathrm{T}_{\mathrm{W}}-\mathrm{T}_{\mathrm{s}}=9.4^{\circ} \mathrm{F}$. Inserting these values into Equation II-17, the predicted growth rate for $b=\pi / 2$ is shown on Figure II-3 also.

To the writer's knowledge no other experimental data for liquids at saturation are available in the Iiterature for further comparison. On the basis of present results it appears that at atmospheric pressure, at low heat flux densities, and for liquids at saturation temperature, Equation II-17 with $b=\pi / 2$ approximates experimental data adequately.

The agreement of Equation II-17 with experimental data indicates some areas for further investigation and offers interesting possibilities. We shall discuss some of them before proceeding with the analysis of the growth and collapse of bubbles in subcooled liquids. 


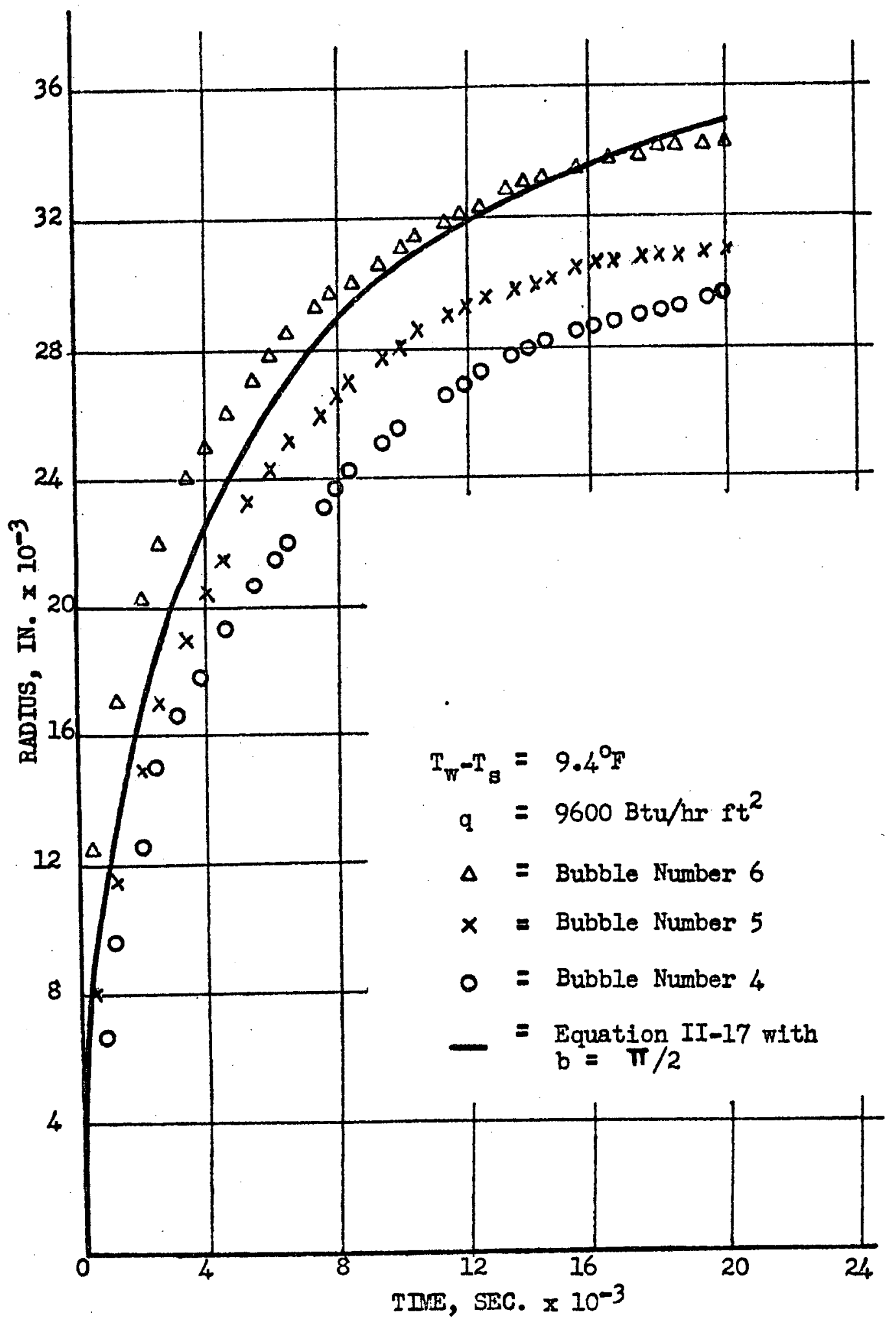

FIGURE II-3. COMPARISON OF EQUATION II-17 WITH ZMOLA'S (42) EXPERIMENTAL DATA FOR BUBBLES IN POOL BOILING OF WATER AT SATURATION TEMMERATURE 
II-4. Maximum Bubble Diameter and the Thiclness of the Superheated Liquid Film

It is seen from Equation II-16 that the bubble will stop growing when

$$
\sqrt{\pi a t}=\frac{k \Delta T}{q}
$$

That is, when the diffusion length becomes equal to the thickness $x_{s}$, of the superheated liquid film adjacent to the heating surface. The maximum bubble diameter is given then by

$$
D_{m}=\frac{\Delta T C P_{L}}{L P_{r}} \frac{K \Delta T}{q}
$$

which is equivalent to:

$$
\frac{D_{m}}{x_{s}}=\frac{\Delta T C P_{L}}{L P_{V}}
$$

This is not surprising since it is in energy balance nil novi sub sole. Consider a surface area $A$, and $a$ liquid film of thickness $x_{s}$, adjacent to it. If this liquid film is superheated by $\Delta T$ degrees the internal energy of the liquid is given by: $\mathrm{Ax}_{s} \Delta \mathrm{TC} \rho_{L}$. If all this stored energy is used for vaporization, the maximum distance the vapor front can advance is given by: 


$$
A D_{m} L P_{V}=A X_{S} \Delta T C P_{L} \quad I I-24
$$

It follows from Equation II-23 that, in boiling, the significance of Jalrob's number is that it represents the ratio of the maximum bubble radius to the thiclmesa of the superheated Iiquid film.

For the experiments of Znola, Equation II-23 predicts a maximum radius $R_{\mathrm{m}}=36.6 \times 10^{-3} \mathrm{in.}$, which would be reached when $R=0$. It is interesting to note that the growth curves on Figure II-3 show almost zero velocity at $t=20 \times 10^{-3} \mathrm{sec}$; indiceting a deficiency of energy available to maintain the vaporization process.

II-5. The Thiclness of the Superheated Liquid Film The nucleation from a cavity was discussed in Section I-2; it was noted that the dimensions of a cavity is important in the nucleation process from a heated surface. The results of the previous section indicated the importance of the thicleness of the superheated liquid film. Both the nucleation and the thicleness of the superheated film affect the dynamics of a bubble. one is lead therefore to investigate whether or not a relation exists between the diameter $D_{c}=2 r_{0}$, of the 
cavity and the thiclness of the superheated film given by

$$
x_{s}=k \frac{T_{w r}-T_{s}}{q}
$$

For the experiments of Griffith and Wallis (16) the superheat which initiated boiling was: $\quad T_{W}-T_{S}=20^{\circ} \mathrm{F}$, and the heat flux was $q=40,000 \mathrm{Btu} / \mathrm{hr} \mathrm{ft}^{2}$. Substituting these values into Equation II-26 the film thicleness becomes $\mathrm{x}_{\mathrm{s}}=2.39 \times 10^{-3} \mathrm{in}$. The diameter of the cavity in the experiments of Griffith and Wallis was $D_{C}=2.7 \times 10^{-3} \mathrm{in}$. Such a close agreement is rather surprising. To explore further this relation the experimental data for pentane and ether reported by clark, Strenge and Westwater (10) are reproduced on the following table together with the corresponding values of $\mathrm{X}_{8}$ computed from Equation II-26. For some pits two values of the superheat temperature were reported; the second was the observed value whereas the first one was corrected to smooth the data. The accuracy of the heat flw density $q$, and of the superheat temperature $T_{W}-T_{S}$, were not evaluated for these experiments. 
TABLE II-3

Comparison of Computed Film Thickness with the Diameters of Active Gavities in Westwater-Strenge's Experiments

Pit

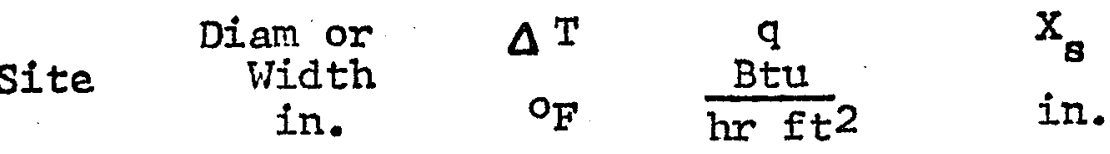

$\begin{array}{rlrrl}1 & 0.0003 & 14 & 13400 & 0.0010 \\ 2 & 0.0003 & 50 & 8100 & 0.0057 \\ 3 & 0.00038 & 23-35 & 12300 & 0.0019-0.0029 \\ 4 & 0.0082 & 12 & 5400 & 0.0021 \\ 5 & 0.0018 & 11-19 & 2600 & 0.0040-0.0068 \\ 6 & 0.0018 & 23-35 & 12300 & 0.0017-0.0024 \\ 7 & 0.0020 & 22-30 & 13000 & 0.0017-0.0023 \\ 8 & 0.0022 & 23-35 & 12300 & 0.0018-0.0029 \\ 9 & 0.0022 & 27-42 & 15600 & 0.0017-0.0026 \\ 10 & 0.0023 & 14 & 4900 & 0.0026 \\ 11 & 0.0025 & 21-31 & 13200 & 0.0015-0.0022 \\ 12 & 0.0026 & 27-41 & 15600 & 0.0017-0.0025 \\ 13 & 0.0033 & 10 & 3400 & 0.0028\end{array}$

Scratch

$\begin{array}{lccrl}1 & 0.00008 & 50 & 8100 & 0.00058 \\ 2 & 0.00018 & 14 & 8900 & 0.0015 \\ 3 & 0.00044 & 23-35 & 12300 & 0.0017-0.0027 \\ 4 & 0.00075 & 13 & 17600 & 0.00069\end{array}$

It is seen from the above table that, with exception of Pits No. 1 and No. 2, the computed thicleness $\mathrm{X}_{8}$, appears to be of the same order as the diameters of active cavities $D_{c}$. For scratches such an agreement is not indicated.

A relation between $D_{c}$ and $x_{s}$ such as that indicated by these experiments offers interesting possibilities in an analysis of boiling heat transfer and initial 
boiling transients. We shall note only one here. Since the diameter of a cavity is a characteristic of the surface, a relation between $D_{c}$ and $x_{s}$ implies that for a given heat flus the local superheat temperature $T_{W}-T_{s}$, is determined by the diameter of the active cavity. In steady boiling at low heat transfer rates, a known distribution of cavities would permit then a derivation of an equation relating the average superheat temperature to the average heat flux.

in view of the indicated relation between the cavity diameter and the thiclness of the superheated liquid film, and in view of the possibilities that this relation offers, it appears that further experimental and theoretical investigations are varranted.

II-6. Implications and Limitations of the Model In this section will be discussed some implications and limitations of the extended Bosnjakovic-Jakob model. In order to bring these implications and limitations into focus we shall describe first the actual physical process. This brief description is based on an excellent discussion of the bubble growth problem given by Ellion (15).

A thin superheated liquid film with a steep temperature profile exists adjacent to the heating surface. Following the nucleation, the bubble starts 
growing in this superheated liquid film. The bubble is, initially, hemispherical; it is probable that the bottom of the bubble is separated from the wall by a thin layer of liquid since the viscous and adhesion forces in the liquid prevent the bubble from wiping the wall clear of water and the evaporation rate may not be sufficiently large to evaporate any appreciable thickness of liquid at the bubble base. Ellion was not able to verify the existence of this thin film because of the limited resolution of the camera lens. In view of the presence of the nucleating cavity, the existence of a "dry" point appears probable also. The superheated liquid film that was initially above the nucleating cavity is pushed away from the wall by the bubble, see Figure II-4. As the bubble grows, the film becomes stretched and this results in higher temperature gradients. Heat is removed from the displaced superheated film by conduction and convection to the bulk liquid and by evaporation at the bubble interface. Evaporation occurs also at the base of the bubble. The rate of evaporation and, consequently, the rate of growth decreases as the superheated film becomes cooler. As the bubble decelerates the momentum that was stored in the liquid and the buoyant force tend to pull the bubble aray from the surface. Because 


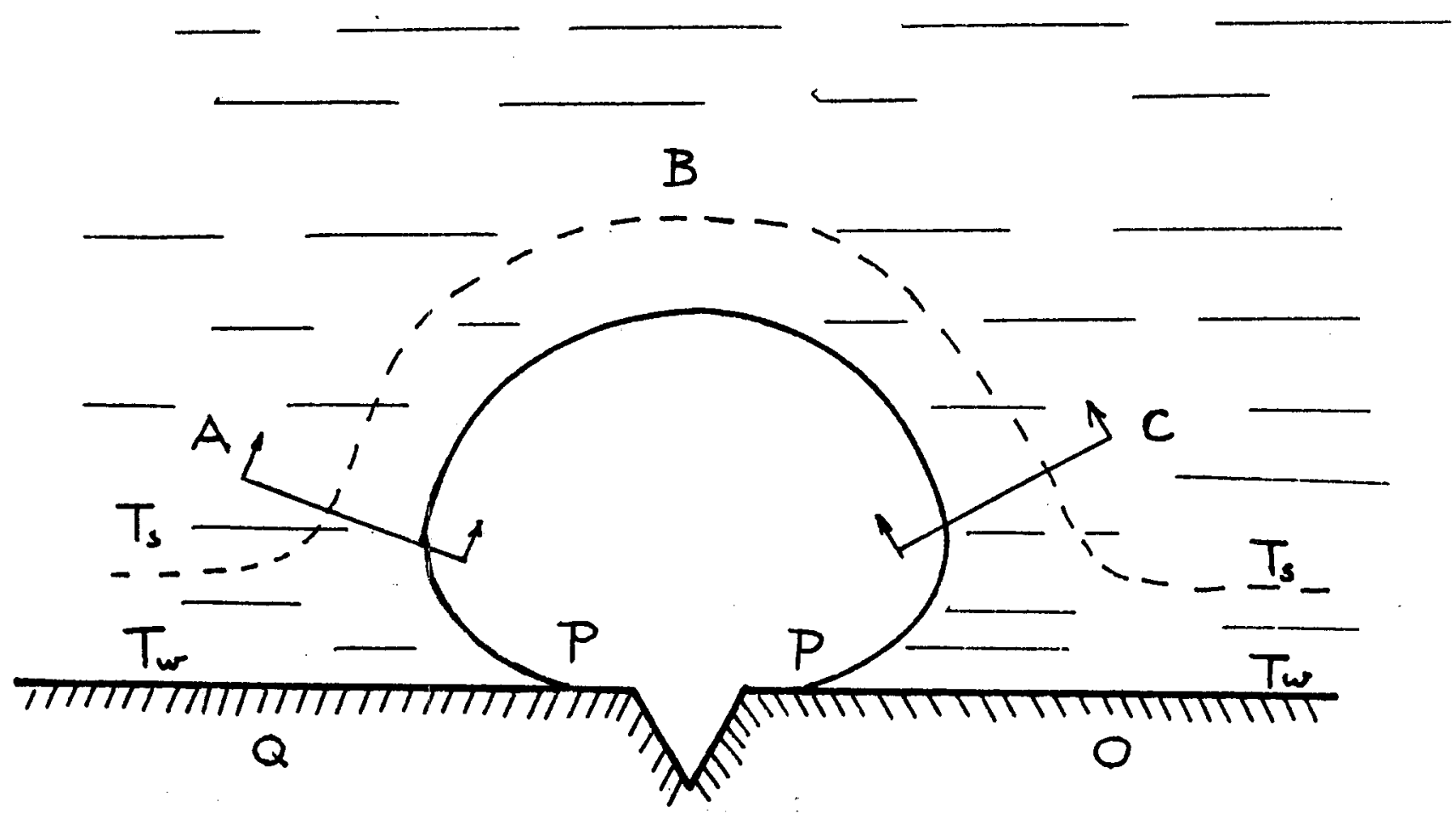

Figure II-4. The Probable Bubble Form and the Approximation of the Model 
of the adhesion forces and the resistance to motion the bubble becomes deformed. If the bulk liquid is at saturation temperature the bubble will depart from the surface; if it is subcooled the bubble will collapse. It is seen from this brief description that a complete analysis of the bubble growth problem on a heated surface should take into account the hydrodynamic aspect as well as the energy aspect of the problem. Since the process takes place in such a vicinity of the solid surface it is apparent that the viscous effects of the liquid as well as the inertial should be retained in the analysis. In addition, because of the liquid-vapor interface and of the solid-liquid-vapor interface, both the surface tension and the contact angle become important. Because evaporation takes place over the entire interface the energy equation should take into account the transfer of energy from the wall to the liquid and the interface in the regions QAP and OCP, during the entire bubble lifetime. As a consequence of this energy transfer the temperature distribution in the solid in the region OPQ will not be uniform. Thus, the problem involves two energy equations, one for the solid and the second for the liquid with a matching problem along an unlenown moving boundary. 
In view of the complexity of the problem any agreement of predicted growth rates by the simple model with experimental data is rather surprising. It can be seen from Figure II-4 that the model considers only the energy transfer in the region $A B C$. The motion of the interface depends only on the amount of stored energy which existed originally in the superheated film.

The limitations of the model now become apparent. The model cannot take into account any temperature change due to heating after the growth has started, i.e., after the vapor has insulated locally the liquid from the solid. Since the regions $Q A P$ and $O C P$ are not considered, the effect of additional heating during the growth process is not accounted for. We note, however, that it appears that this restriction could be removed; it will be discussed in a future report. Because the hydrodynamic effects were not considered, the effect of the distortion of the bubble on the temperature field is neglected.

It is seen from these limitations that if any agreement is to be expected the effect of evaporation in the regions QAP and OCP should be small when compared to the evaporation in the region $A B C$. To achieve this, two conditions must be satisfied. First, the transfer area in the regions QPA and OPC should be 
small in comparison with the transfer area $A B C$. Second, the rate of vaporization in the region $A B C$ should be fast so that any change of the temperature field in the regions QPA and OPC, caused by heating during the growth process is small. The effect of the bubble distortion on the process of vaporization in the region $A B C$ will be small if the distortion occurs at the end of the growth process. In view of the agreement of predicted growth rates with experimental data it appears that either these conditions were satisfied, or that the effects of various assumptions tend to compensate each other.

Another important limitation should be noted before closing this section; it is concerned with the "thin thermal boundary layer" assumption. It was noted in Section II-I that for a growing bubble this assumption is valid when the Jakob number is larger than unity. The assumption will be satisfied also for a collapsing bubble as long as the thickness of the thermal boundary layer is smaller than the bubble radius (see Figures II-1 and II-2). For sufficiently small radii, hovever, Equation II-12 indicates that an equation which is based on the "thin boundary" assumption will underestimate the collapse rate. This question appears to be important for bubble dynamics in subcooled liquids, a topic which 
is taken up in the next section.

II-7. Dynamies of a Vapor Bubble in a Subcooled Liquid Bubble dynamics in a subcooled liquid was investigated experimentally by Gunther and Kreith (7) and by Ellion (15) in a non-flow system, and by Gunther (43) and by Treshov (41) in a flow system. The problem was investigated also analytically be various researchers. The growth problem in a non-uniform temperature field was analyzed by Griffith (44) and by Savic (39). An analysis of a bubble growing and collapsing in a subcooled liquid was reported by Bankoff and Mikesell $(45,46)$. The time needed for a bubble to reach the maximum radius before collapsing was considered by Forster (47). The collapse of a bubble in a uniformly subcooled liquid was analyzed by Plesset and Zwick (48). The condensation rate of a vapor bubble which is introduced in a subcooled liquid was discussed by the writer (49). In the following, the growth and collapse rates predicted by the extended Bosnjalcovic-Jakob model will be compared to the experimental results of Gunther and Kreith and of Ellion.

Ellion's experiments were performed with water at atmospheric pressure; Figures 47, 49,53 and 57 from Ellion's dissertation are reproduced in this Chapter as Figures II-5, 6, 7 and 8 . Figures II-5 and II-6 
show the effect of increasing the heat flux density from $5.29 \times 10^{5} \mathrm{Btu} / \mathrm{hr} \mathrm{ft}^{2}$ to $11.5 \times 10^{5} \mathrm{Btu} / \mathrm{hr} \mathrm{ft}^{2}$ at a constant bull Iiquid temperature $T_{L}=135^{\circ} \mathrm{F}$, i.e., at a constant subcooling of $T_{S}-T_{L}=77^{\circ} \mathrm{F}$. Whereas Figures II-7 and II-8 show the effect of increasing the bulk liquid temperature from $T_{I}=78^{\circ} \mathrm{F}\left(T_{S}-T_{L}=134^{\circ} \mathrm{F}\right)$, to $T_{I}=177^{\circ} \mathrm{F}\left(T_{s}-T_{L}=35^{\circ} \mathrm{F}\right)$, at a constant heat flux density of $7.79 \times 10^{5} \mathrm{Btu} / \mathrm{hr} \mathrm{ft}^{2}$. It can be seen from these figures that a given average heat flux and average temperature variations occur in the dynanics of different bubbles. Ellion noted that, because of the se large variations, an agreement closer than $25 \%$ should not be expected. The large changes which are indicated by these figures point to the importance of local conditions which exist in the vicinity of a nucleating center and, consequently, to the statistical nature of the overall process when an ensemble of nucleating centers, i.e., of bubbles, is considered.

We shall compare now the growth and collapse rates predicted by Equation II-17 with Ellion's experimental data. It is seen from Equation II-16 that the maximum radius $R_{m}$ is reached when, 
PIGURE II-5. ELLION'S EXPERTMENTAL DATA (RETERENCE 15)

FOR BUBBLES GROITING AND COLLAPSING IN SUBCOOLED TATER

$$
q=5.29 \times 10^{5} \mathrm{Btu} / \mathrm{hr} \mathrm{ft}^{2}, \mathrm{~T}_{L}=135^{\circ} \mathrm{F}, \mathrm{T}_{8}-\mathrm{T}_{L}=77^{\circ \mathrm{F}}
$$

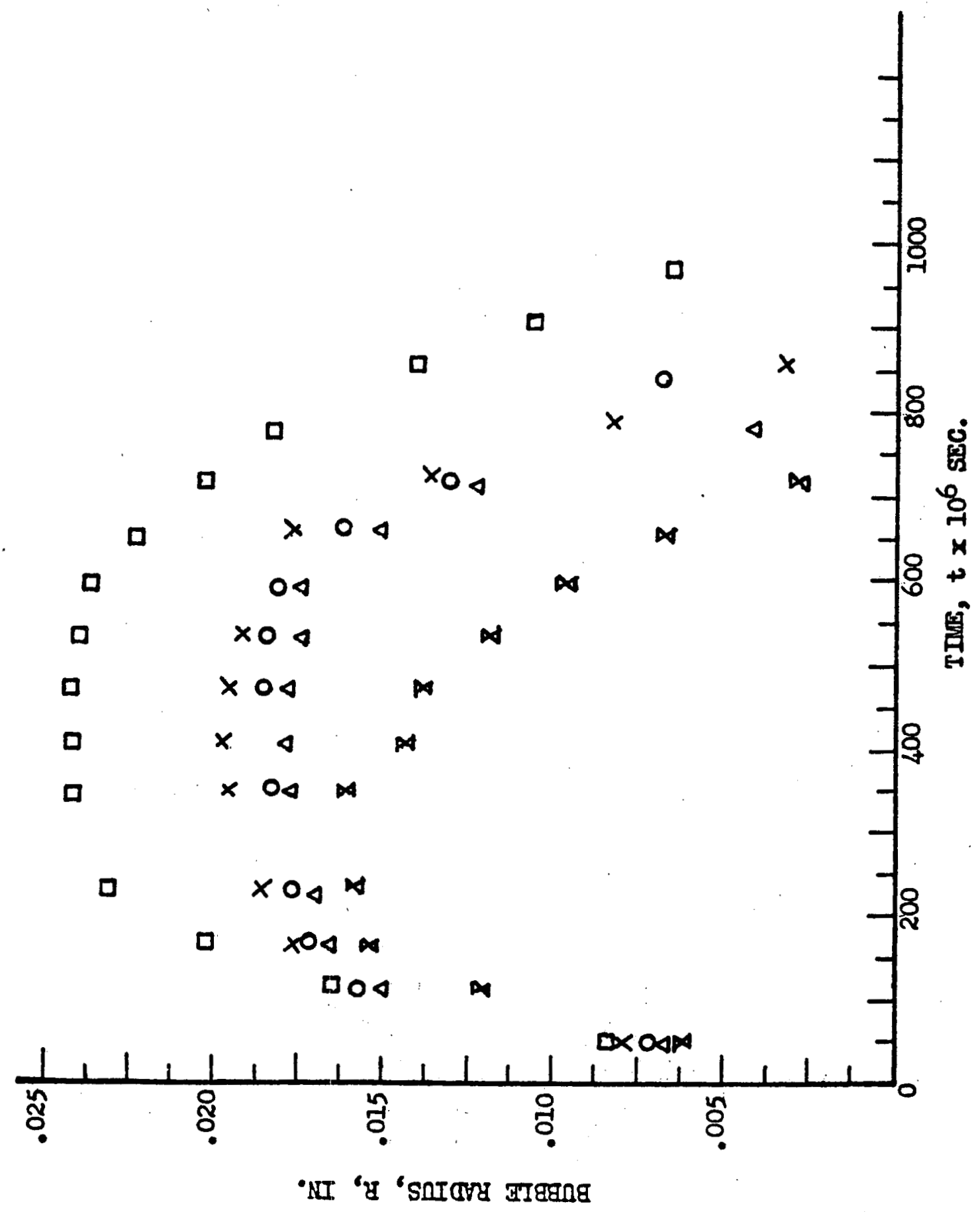


FIGUEE II-6. ELIION'S EXPERDIENTAL DATA (REFERENCE 15) FOR BUBBLTS GROWING AND COLLAPSING IN SUBCOOLED TATER

$q=11.5 \times 10^{5} \mathrm{Btu} / \mathrm{hr} \mathrm{ft}, \mathrm{T}_{L}=135 \mathrm{~F}, \mathrm{~T}_{\mathrm{s}}-\mathrm{T}_{\mathrm{L}}=77^{\circ \mathrm{T}}$

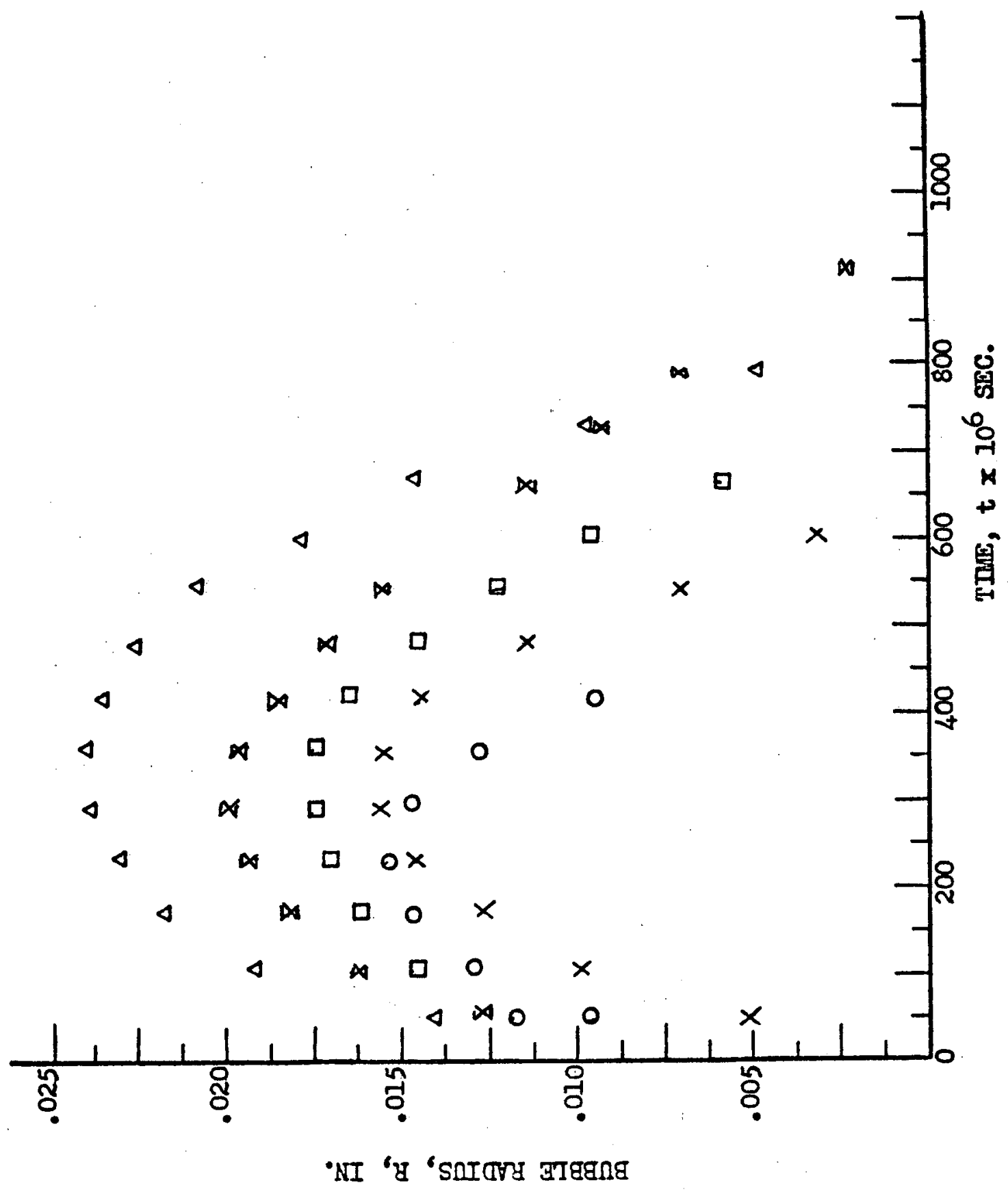


FIGURE II-7. ELLION'S EXPERDIENTAL DATA (REFERENGE 15)

FOR BUBBLES GROWING AND COLLAPSING IN SUBCOOLED TATER

$$
q=7.79 \times 10^{5} \mathrm{Btu} / \mathrm{hr} \mathrm{ft}^{2}, \mathrm{~T}_{\mathrm{L}}=78 \mathrm{O}_{\mathrm{F}}, \mathrm{T}_{\mathrm{s}}-\mathrm{T}_{\mathrm{L}}=134_{\mathrm{F}}
$$

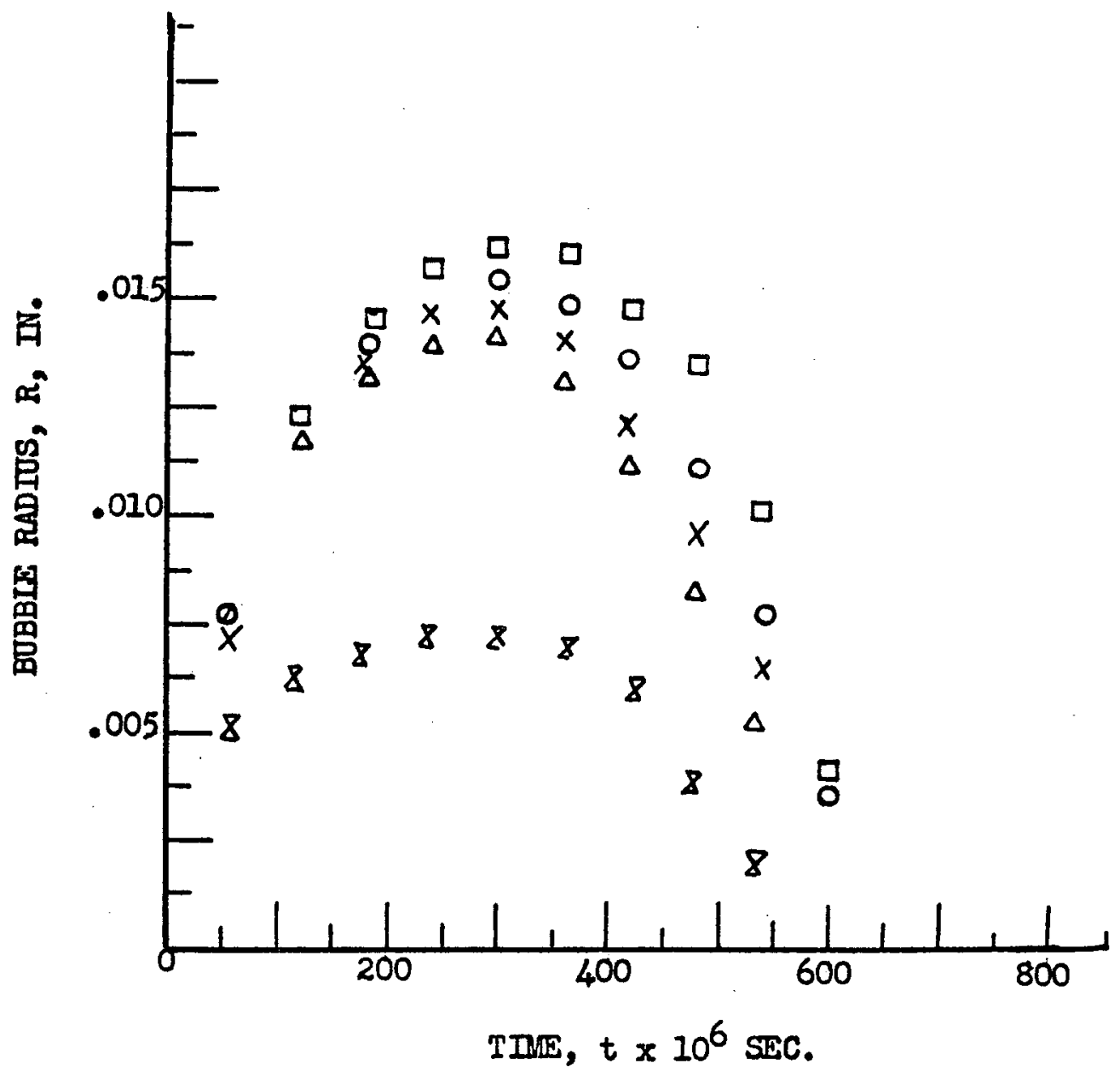


FIGURE II-8. ELLION'S EXPERDIENTAL DATA (REFERENCE 15)

FOR BDBBLES GROTING AND COLLAPSIIG IN SUBCOOLED TATER

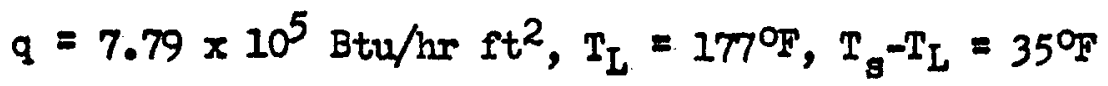

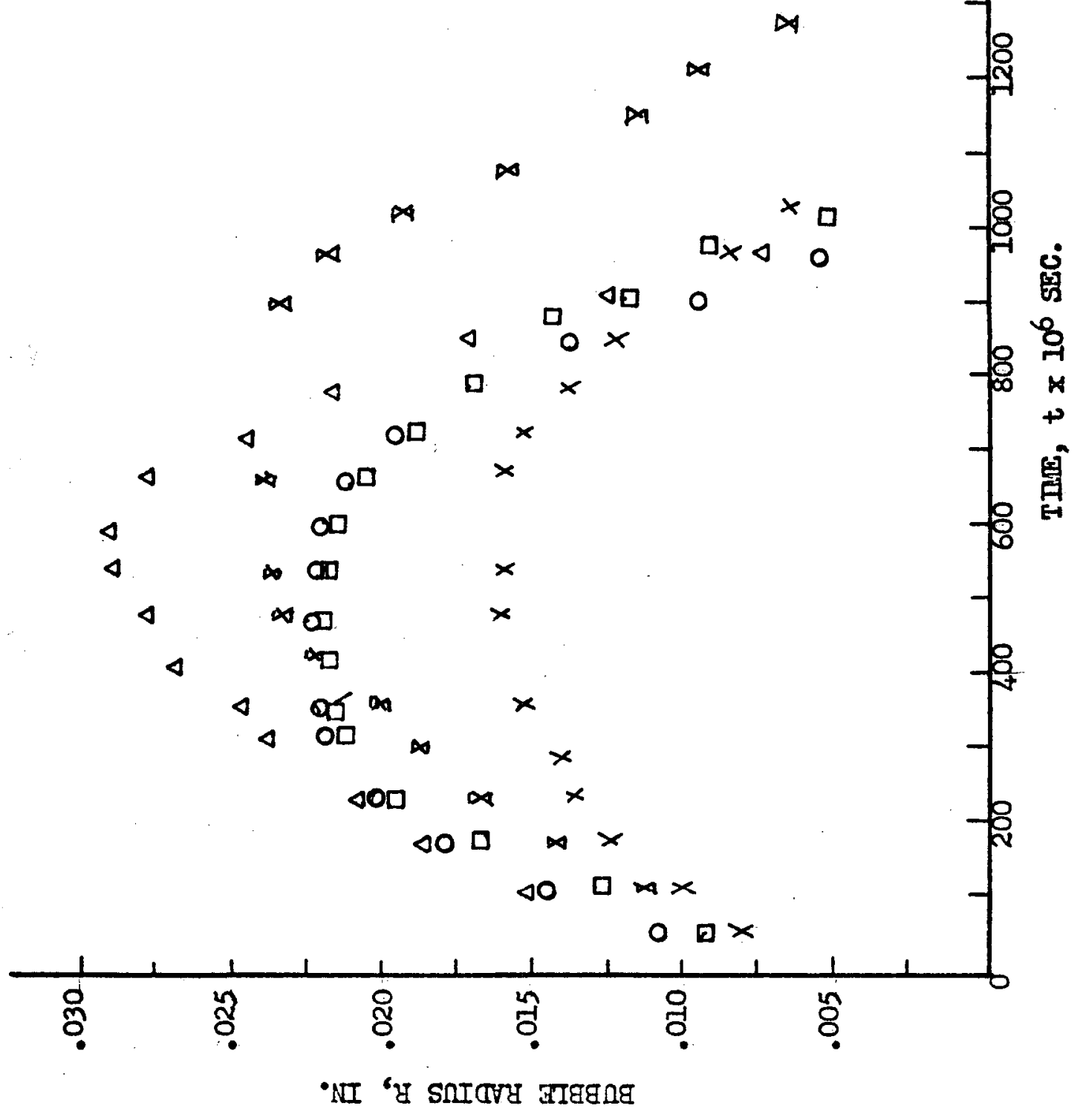


at

$$
\sqrt{\pi a t_{m}}=k \frac{T_{w}-T_{s}}{q}
$$

The radius vs. time relation given by Equation II-17 can be expressed in terms of the maximum growth time $t_{m}$, thus

$$
R=b \frac{2}{\pi} \frac{\left(T_{w}-T_{s}\right) C P_{L}}{L P_{V}}\left[1-\frac{1}{2} \sqrt{\frac{t}{t_{m}}}\right] \sqrt{\text { rat }}
$$

The maximum bubble radius is then given by:

$$
R_{m}=\frac{b}{\pi} \frac{\left(T_{w}-T_{s}\right) c r_{L}}{L \rho_{v}} \sqrt{\pi a t_{m}}
$$

It is advantageous to introduce a dimensionless radius given by $R / R_{m}$ and a dimensionless form is obtained from Equation II-28 and II-29, thus

$$
\frac{R}{R_{m}}=\sqrt{\frac{t}{t_{m}}}\left[2-\sqrt{\frac{t}{t_{m}}}\right]
$$

Equation II-30 is plotted on Figures II-9, 10, 11 and 12 together with Ellion's data show on Figures II-5, 6 , 7 , and 8 respectively. The advantage of nornalizing the data becomes apparent. 


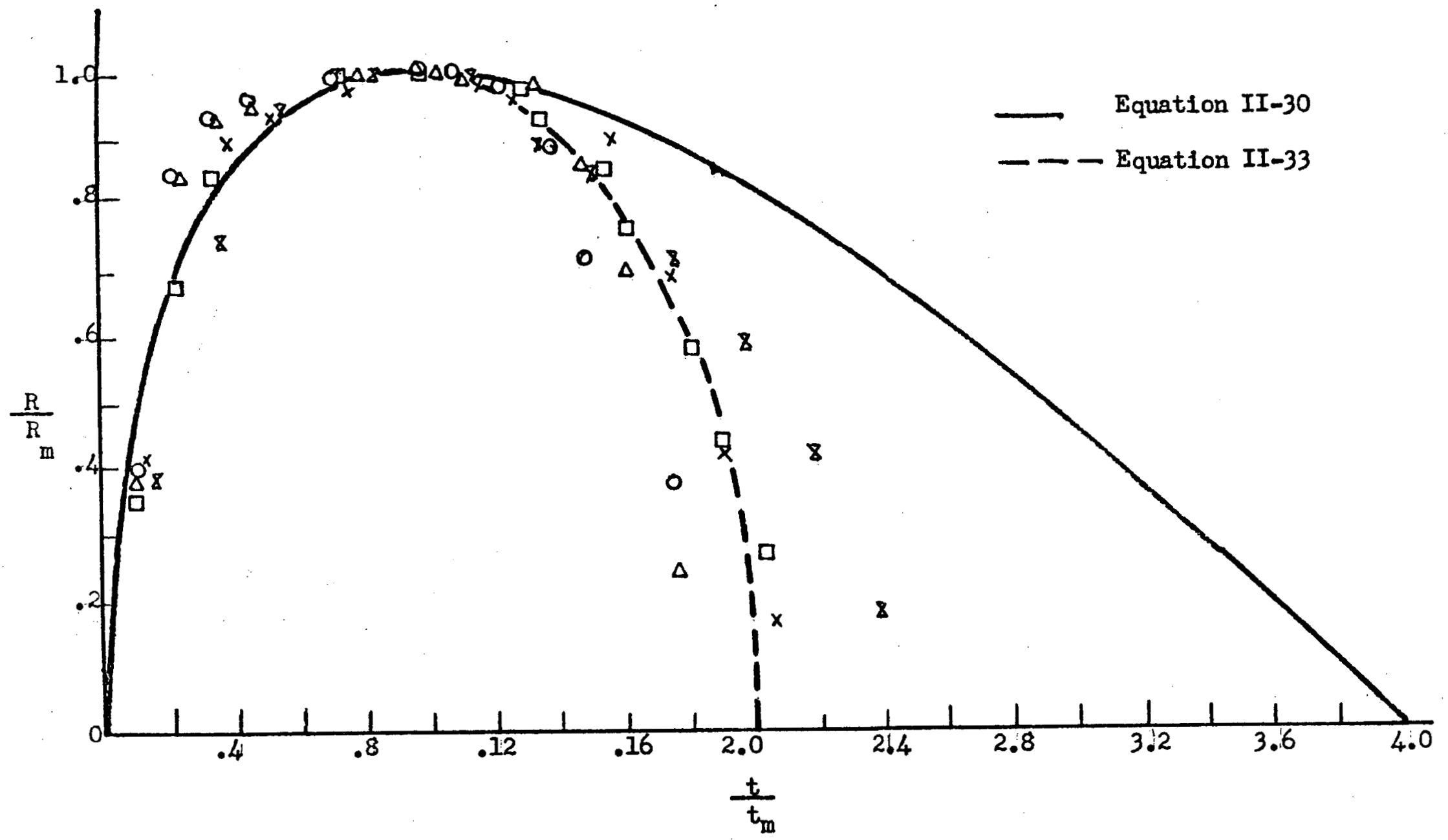

FIGURE II-9. COMPARISON OF EGUATION II-30 and II-33 WITH ELLION'S EXPERTIENTAL DATA SHOHN ON FIGURE II-5 


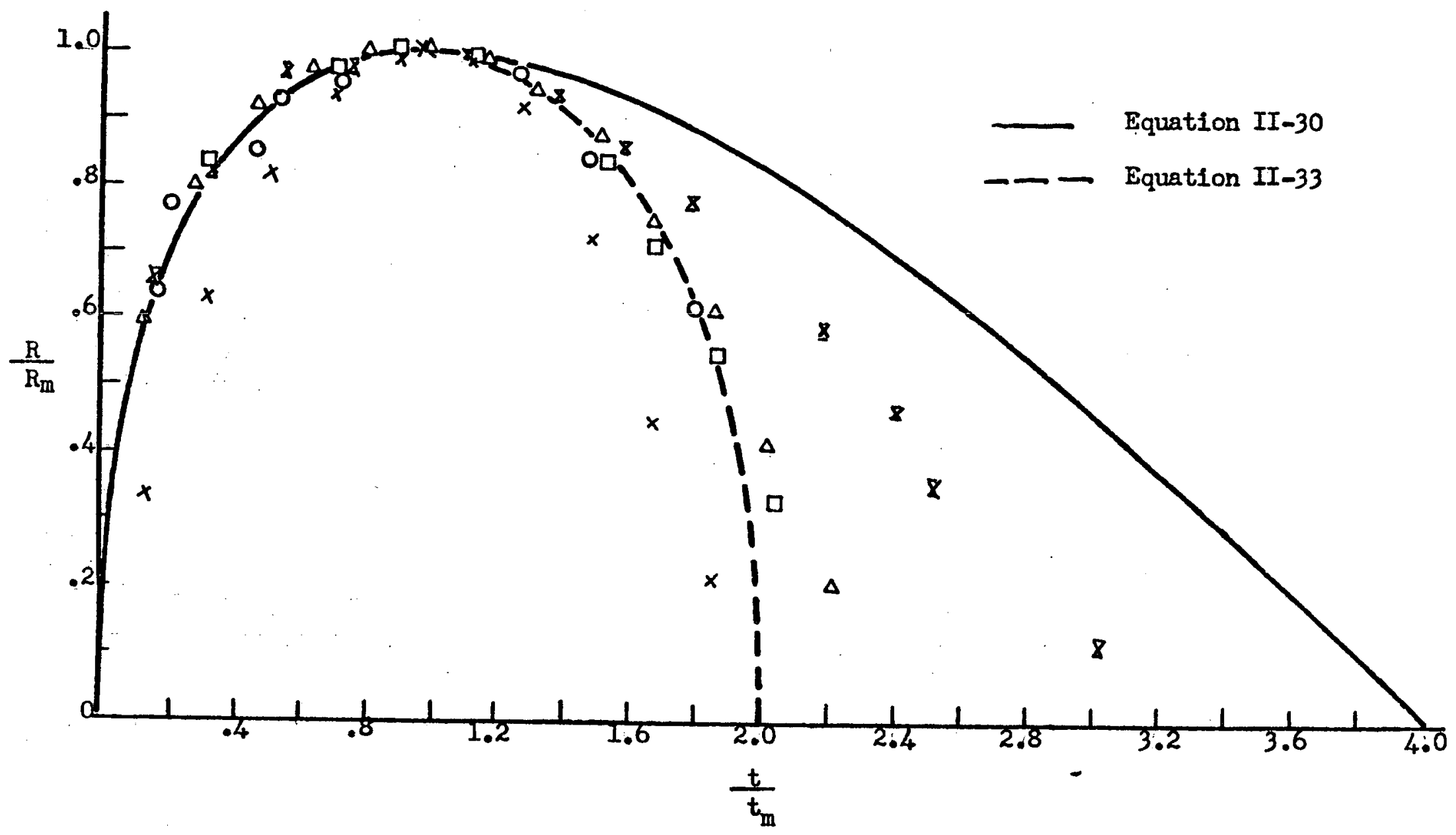

FIGURE II-10. COMPARISON OF EQUATION II-30 AND II-33 WITH ELLION'S EXPERDENTAL DATA SHOWN ON FIGURE II-6 


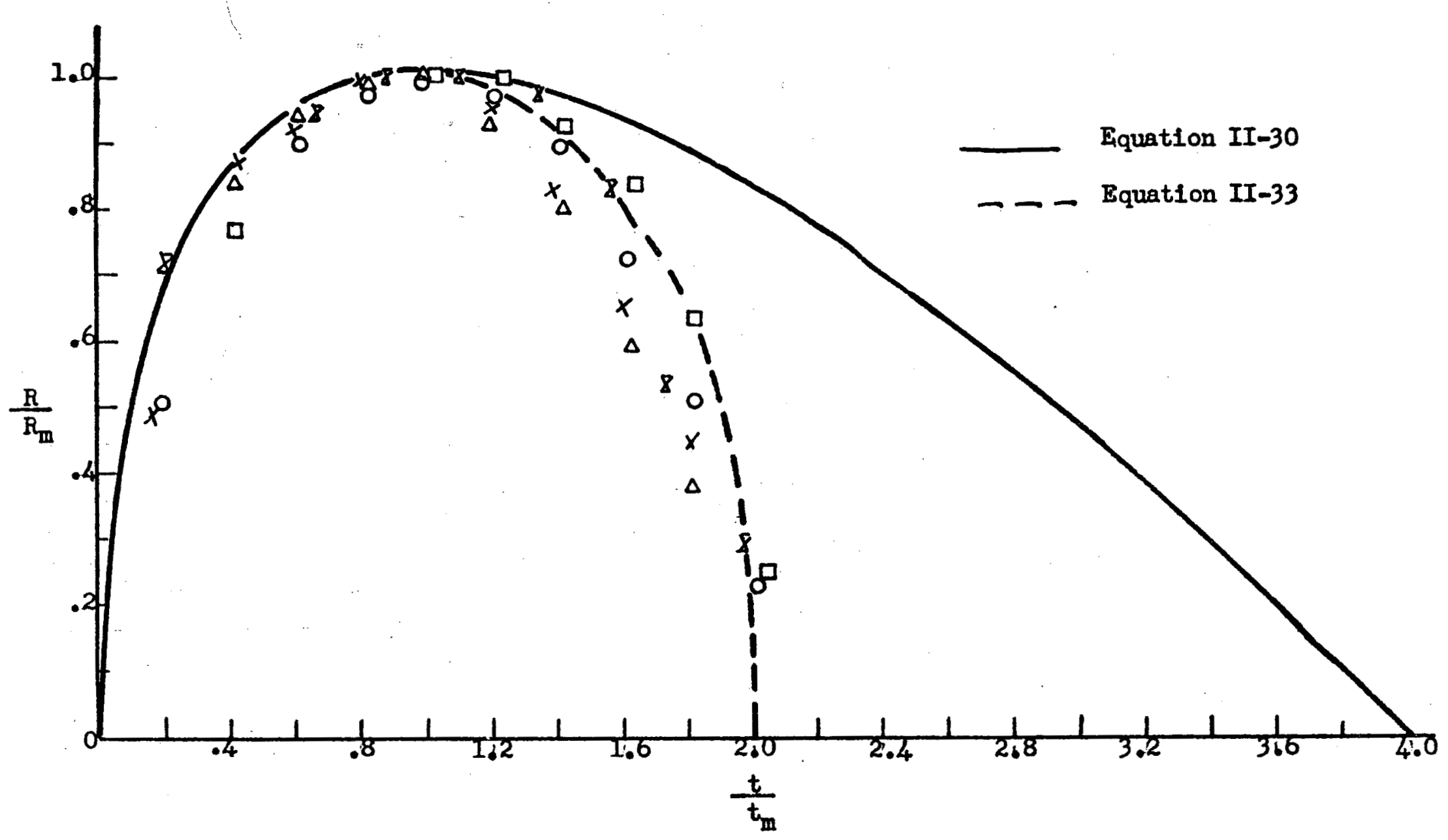
FIGURE II-11. COMPARISON OF EQUATION II-30 AND II-33 WITH ELLION'S
EXPERTENTAL DATA SHOWN ON FIGURE II-7 


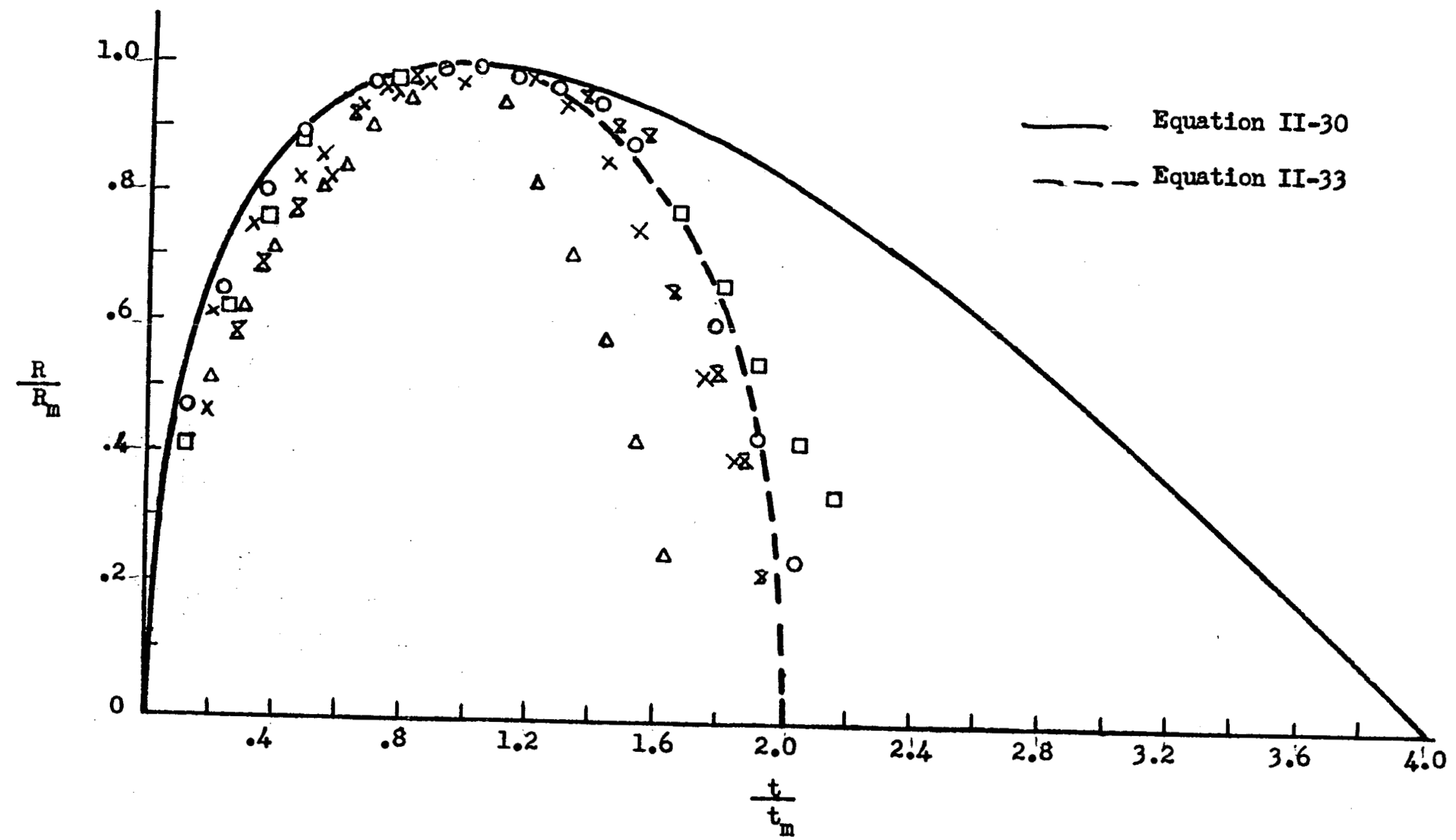

FIGURE II-12. COMPARISON OF EQUATION II-30 AND II-33 WITH EILION'S EXPERTIENTAL DATA SHOFIN ON FIGURE II-8 
It appears from the se figures that Equation II-30 adequately approsimates the growth process. It is seen, also, that the model is not adequate for predicting the collapse stage. The reason for this inadequacy can be understood in terms of the discussion of the preceding section.

The inertia of the liquid and the buoyant force tend to pull the bubble away from the surface, causing a deformation of the bubble after it reaches the maximum radius. This defornation and motion of the bubble increase the thermal gradients causing a faster condensation. As the vapor condenses the thermal boundary layer increases, whereas the radius decreases. Consequently, the "thin boundary layer approximation" becomes progressively worse. Because the curvature increases with a decrease of radius, it is seen from Equation II-12 that the thermal gradients increase enabling faster condensation rate than predicted by the thin boundary approximation. Heat transfer and consequently, the condensation rate during the collapse is enhanced also by the turbulence of the liquid, as noted already by Griffith (44) and by Bankoff and Mikesell (45). Let us examine now the significance of these favorable heat transfer conditions. As the vapor condenses at the bubble interface latent heat is liberated and the 
temperature of the interface increases. Unless this energy is transferred to the bulk liquid the rate of condensation will decrease. High heat transfer rates to the bulk liquid thus minimize the temperature variations at the interface and in the vapor due to the latent heat. Thus, when large thermal gradients are present it appe ars permissible to consider, in the limit, an isothernal collapse process. For an isothermel process the inertia of the liquid becomes important.

It was shown already by Plesset and Zwick (48) that, for bubbles collapsing in a liquid at uniform temperature, the thermal effects on restricting the collapse rate are unimportant. Consequently, only the inertial terms in Rayleigh's equation of motion are of significance. The inertial effects were used by Bankof $f$ and Mikesell (45) to explain both the grawth and the collapse of a bubble in a subcooled liquid. We shall examine the inertia effect in terms of the extended Bosnjakovic-Jakob model.

Assuming spherical sytunetry, the liquid inertia can be taken into account by combining Rayleigh's equation of motion, Equation II- $\dot{6}$, with the energy equation. As an analogy to the bubble growth problem in a uniformly superheated liquid, the bubble growth and collapse equation for a subcooled liquid becomes: 
$R \ddot{R}+\frac{3}{2} \dot{R}^{2}+\frac{2 \sigma}{P L R}=\frac{1}{R} \frac{d P}{d T}\left[T_{\omega}-T_{S}-\frac{L P_{v} \dot{R} \sqrt{\pi a L}}{K b}-\frac{9 \sqrt{\pi a t}}{K}\right] I I-31$

where the term $\mathrm{dP} / \mathrm{d} T$ is given by Clausius-Clapeyron equation. With the term $q=0$, and $b=\pi / 2$, Equation II-3I is reduced to the equation which describes the growth of a bubble in a uniformly superheated.

liquid; an equation which was analyzed already in References 32,33 and 34,35 .

During the growth process, the effects of the inertia and of the surface tension are small in comparison with the heat transfer effect. Consequently, during the growth the left hand side can be neglected, and Equation II-3I is reduced to Equation II-17. As the bubble radius goes through the maximum, the right hand side of Equation II-3I changes signs and becomes negative. In this domain then, the liquid inertia cannot be neglected. It was noted already that, because of the combined effects of liquid turbulence and of bubble curvature, the collapse stage could be approximated by an isothermal process. Neglecting the effect of surface tension, Rayleigh's equation for an isothermal process becomes : 


$$
R \ddot{R}+\frac{3}{2} \dot{R}^{2}=-\frac{\Delta P}{P_{L}}=\text { const. }
$$

The solution of this equation for the initial conditions given by: $t=0, R=R_{m},: \dot{R}=0$, can be obtained from Lamb (50), thus

where:

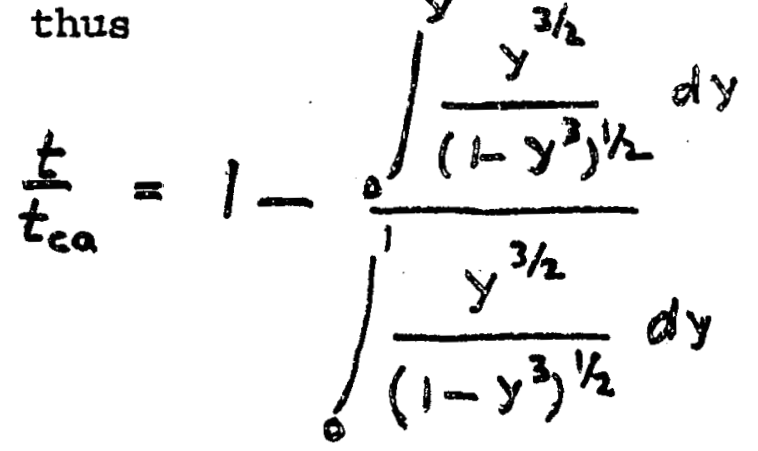

$$
y=\frac{R}{R}
$$

and $t_{c}$ is the time needed for the total collapse. Equation II-33 is plotted on Figure II-9, 10, 11 and 12; the initial time for collapse was taken to correspond to $t_{\mathrm{m}}$, in order to satisfy the initial conditions. The agreement appears satisfactory. Note that the large variations in experimental data shown on these figures point out the poor reproducibility of the data and the statistical nature of the process.

In closing, we note that if a bubble is suddenly introduced in a subcooled liquid, or if the saturation temperature of the liquid is suddenly increased (by increasing the pressure), then, initially, the thickness of the thermal boundary layer is much smaller than the bubble 
radius. Under these conditions, as discussed in Reference 49, the initial collapse rate will be given by:

$$
L P_{v} \stackrel{\circ}{R}=-\frac{\pi}{2}-\frac{T_{s}-T_{1}}{\sqrt{T_{t}}}
$$

We shall summarize now the results presented in this chapter.

1) It appears that experimental data for bubbles growing and collapsing in a subcooled liquid can be approximated by considering the growth and collapse process separately.

2) The growth process is governed by the rate of heat transfer to the bubble wall. The esperimental data can be approsimated by growth rates obtained from the extended BosnajakovicJakob mode1.

3) The collapse stage can be approxinated by the solution of Rayleigh's equation for an isothermal process.

4) The growth and collapse equations thus obtained are matched at the maximum bubble radius.

5) The large variation of experimental data point out the poor reproducibility of the data and the statistical nature of the process.

6) Experimental data tend to indicate that the diameter of an active cavity can be related to the thicleness of the superneated liquid film. 
CHAPTER III

HYDRODYNAMIC ASPECTS OF NUCLEATE BOILING

Two aspects of bubble dynamics, the nucleation and the growth, were discussed and analyzed in the preceding chapters. It was seen that the growth equation can predict the dynamics of a bubble while it is still attached to the surface. However, the analysis cannot predict when and how the bubble will depart from the surface. The mechanist of nucleate boiling was discussed in Chapter I. It was noted that growing and departing bubbles agitate the superheated liquid film; thus, colder liquid is brought in contact with the heating surface resulting in high heat transfer rates. Since this renewal rate depends upon the rate at which bubbles depart from the surface, the heat transfer rates in nucleate, pool boiling will depend upon the mechanism which governs the departure of bubbles. This mechanism of heat transfer and the dependence of the heat flux upon the frequency of bubble emission was described and used by Jalkob and co-workers $(2,3,4,5)$ in their analysis of nucleate boiling. It was talken into account also in the studies reported by Rohsenow $(51,52)$, 
Kutateladze (53), Kruzhilin (54, 55) and Sterman (56, 57), among others. Indeed, an analysis which would omit from consideration the mechanism of vapor removal from the heated surface can be considered as incomplete. This statement is supported by the recent experiments of Siegel and Usiskin (58) which were conducted in a gravity-free field. The importance of the gravity in removing the vapor from the surface and thus its influence on nucleate, pool boiling was clearly demonstrated.

In this chapter we shall analyze the problem of vapor removal. It will be seen that the phenomenon of a bubble growing from a nucleating center, and its subsequent departure fror the horizontal surface bears a strong similarity to a gas bubbling frorn a horizontal orifice. This similarity and some known facts concerning the frequency of bubble emission from an orifice will permit an estimate of the frequency of bubble generation in nucleate pool boiling. It will be seen also that the hydrodynamic aspects of nucleate pool boiling are similar to those which characterize the process of a gas bubbling through a porous plate. This similarity, and the hydrodynamic instability (usually referred to as "flooding"), which occurs when a gas is forced to bubble through a perforated plate or a sieve-tray, will help us gain an 
understanding of the basic nature of the phenomenon which induces the change from nucleate to transitional boiling and thus creates the critical heat flux in boiling heat transfer.

III-1. The Similarity Between Bubble Formation at an Orifice and Bubble Formation in Nucleate Boiling The problem of bubble formation at a submerged orifice has received much attention. For detailed information concerning this problem the reader is referred to the studies reported by Krevelen and Hoftijzer (59), Siemes $(61,62)$, Hughes and co-workers (63), Davidson and Amick (64) and Silberman (65), among others.

Seimes (61, 62) and Davidson and Amick (64) presented a detailed study of the regimes of bubbie formation from a given orifice as a function of the gas flow rate. In what follows we shall summarize their results; the quoted sections are taken from Reference 64 .

Static Bubbling Region. At very low gas flow rate the bubble formation is a problem of hydrostatics. The diameter of a bubble can be determined by considering the balance of the buoyant and adhesion forces at the orifice. Denoting by $r_{0}$, the radius of the orifice, the bubble diameter is given approximately by 


$$
D_{d}=\left[\frac{12 r_{0} \sigma}{g\left(p_{L}-p_{v}\right)}\right]^{1 / 3}
$$

Davidson and Amick noted that stationary bubbles could not be formed at orifices larger than about $0.7 \mathrm{~cm}$. In Chapter IV we shall see the reason for this. In this regime the bubble diameter is independent of the gas flow rate. The frequency of bubble emission Increases with the gas flow rate. Bubbles rise with constant velocity. "Therefore for a given orifice the center-tocenter spacing of the rising bubbles is inversely proportional to their frequency. At low frequencies the Iiquid at the orifice may be assumed to come to rest before each bubble escapes. The bubbles are identical at any given point early in their rising path."

Transition Region. "At higher flow rates the bubble volume increases sharply as the frequency levels of the spacing between bubbles has decreased, and each forming bubble is affected by the presence of the preceding bubble, perhaps through the mechanism of a liquid vortex at the orifice. The bubbles now start to form into pairs although still forming individually at regular time intervals at the orifice. A bubble will appear to hover 
above the orifice until the next bubble emerges, and the two bubbles will remain together as they rise. This pattern is repeated by the next two bubbles, and so on indefinitely."

Inciplent Coalescence. "As the flow rate further increases, the members of a bubble pair touch each other. This condition may be called 'doublet formation'. The first member of the doublet usually assumes a hemispherical shape, and the second bubble is elongated vertically; the assembly resembles a mushroom."

Coalescence. "Further flow-rate increase causes the second bubble to penetrate the first one appreciably. Under other conditions the first bubble absorbs part of the second and leaves the residue as a small satellite."...

Coalescence at the Orifice. "Coalescence takes place closer and closer to the orifice as the flow rate Increases. A flow rate is finally reached at which the bubbles coalesce right at the orifice, the first bubble having no time to rise before the second emerges. Under the se conditions it will appear that the frequency of bubbles rising from the orifice has suddenly been halved," Double Coalescence. "As the flow rate further increases, the large coalesced bubbles themselves undergo coalescence as they rise. This may be called 'double coalescence.' 
At this stage the fluid is usually quite turbulent and the gas seems to issue from the orifice as a continuous jet."

The reader should compare now the above description to the discussion presented in section I-3. Indeed, if the expression "increasing the gas flow rate" were changed to "increasing the heat flux density", it would appear as if Davidson and Amick were describing the bubble formation in Yamagata and Nishilkawa's experiments of nucleate boiling, see Figure I-5.

It appears thus that both the types of bubbles and the bubble formation regimes in nucleate bolling and in gas bubbling through a submerged orifice are hydrodynamically similar.

In the section which follows we shall make use of this apparent similarity to predict the frequency of bubble emission in nucleate boiling.

III-2. The Frequency of Bubble Enission and the Value of the Product: Bubble Diameter Times Frequency in Nucleate Boiling

It was noted in section I-3 that during the departure bubbles deform. The rate of rise of bubbles was analyzed by Pebels and Garber (65). The velocity of rise for deformed bubbles was found to be constant and independent of the size. It was found that the data 
for sixteen liquids could be described within $\pm 3 \%$ by the relation

$$
u_{\infty}=1.18\left[\frac{\sigma q\left(\rho_{L}-e_{V}\right)}{P_{b}^{2}}\right]^{1 / 4}
$$

This equation was derived analytically by Frank-Kamenetskii also (66). During the process of quasi-static bubble formation, "simple bubbles" depart under the action of gravity only. It can be expected that, under these circumstances, the velocity of rise in the vicinity of the heated surface will be approximately equal to Equation III-2. Before proceeding further we shall verify this statement. Jalcob $(3,67)$ has reported that the velocity of rise of a bubble immediately after departure is $U_{\infty}=17 \mathrm{~cm} / \mathrm{sec}$. For water at saturation temperature, Equation III-2 predicts a velocity of rise

$$
U_{\infty}=18.6 \mathrm{~cm} / \mathrm{sec} \text {. }
$$

Jakob and Iinke (4) found that while the bubble still adhers to the surface its center of gravity rises with almost the same velocity with which the bubble later rises when it leaves the surface. This observation implies that the following relation is approximately valid 


$$
\frac{D_{d}}{t_{d}}=U_{\infty}=1.18\left[\frac{\sigma g\left(P_{L}-P_{V}\right.}{P_{b}^{2}}\right]^{1 / 4} \quad \text { III-3 }
$$

It follows then from Equation I-9 and Equation III-3 that

$$
D_{d} \cdot f=\frac{t_{d}}{t_{c}+t_{d}} \cdot 1.18\left[\frac{\sigma g\left(\rho_{L}-P_{V}\right.}{\rho_{L}^{2}}\right]^{1 / 4} \quad \text { III-4 }
$$

The maximum frequency of bubble emission occurs when bubbles touch each other, i.e., when the contact time $t_{c}$ is zero. For a spectrum of diameters $D_{d}$, Equation III-4 then gives the corresponding frequency spectrum.

The product $D_{d} \cdot f$ appears in almost every analysis of nucleate boiling $(4,5,51,52,53,54,55,56,57)$. It was discussed in Section I-3 that this product was a constant for a nucleating center. For different liquids the average value of $D_{d}$.f was found to be of the same order of magnitude. Consequently, Jakob and Linle (5) proposed that a constant value of $\mathrm{D}_{\mathrm{d}} \cdot \mathrm{f}=77 \mathrm{~mm} / \mathrm{sec}$ be assigned to this product, whereas Fritz and Ende proposed a value of $95 \mathrm{~mm} / \mathrm{sec}$. We shall compute this value now. Jakob and co-workers $(2,3,4,5,24,67)$ have observed that in their experiments the contact time $t_{c}$ was almost equal to the time at departure $t_{d}$. Taking 
$t_{c}$ equal to $t_{d}$, Equation III-4 becomes

$$
D_{d} \cdot f=\frac{1.18}{2}\left[\frac{\sigma g^{2}\left(e_{2}-e_{d}\right)}{e^{2}}\right]^{1 / 4}
$$

III-5

For water at saturation at one atmosphere Equation III-5 gives a value of $93 \mathrm{~mm} / \mathrm{sec}$, whereas for methanol and carbon tetrachloride it predicts $73 \mathrm{~mm} / \mathrm{sec}$ and $64 \mathrm{~mm} / \mathrm{sec}$ respectively. On Figure III-1, which is reproduced from References 3 and 67 , Equation III-5 (for water) is compared with experimental data of Jakob and co-workers. On the same figure are plotted experimental data of Yamagata and Nishilkava (27) and of Westwater and co-workers $(1,25)$. The agreement appears to be satisfactory.

III-3. A Possible Relation Between the Diameter of a Quasi-Static Bubble and the Diameter of a Cavity

The indicated similarity between bubble formation at orifices and bubble formation in nucleate bolling offers an interesting possibility for further investigation. It was noted that for low gas flow rates the diameter of a departing, quasi-static bubble was given by Equation III-1. It was observed in Section I-3 that at low heat transfer rates in nucleate boiling the bubble formation can be considered as a quasi-static process. If the bubble groving from a nucleating center is 


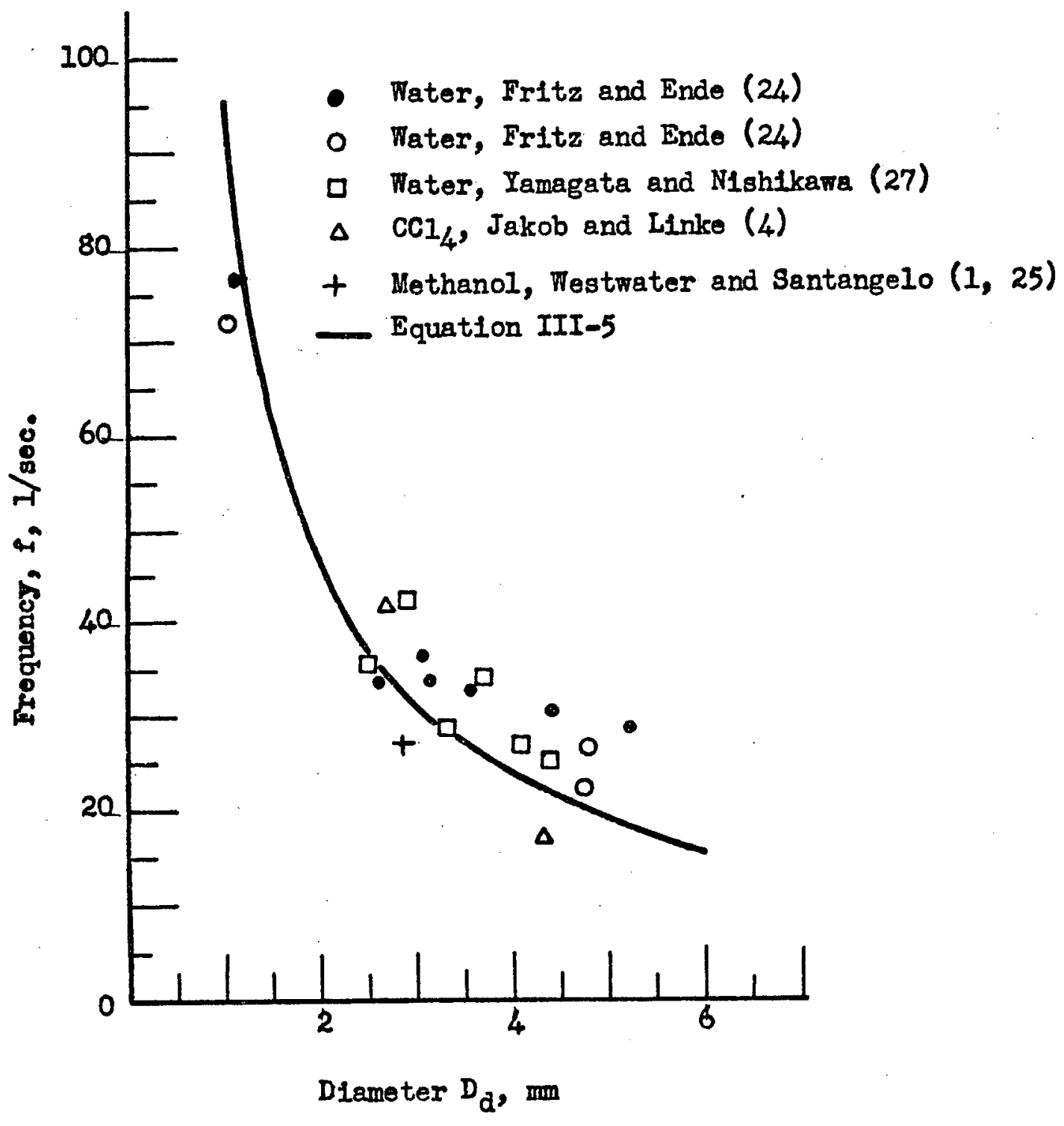

FIGURE III-1. THE RELATION BETWEEN THE FREQUENCY OF BUBBLE EMISSION AND THE DIAMETER OF A BUBBLE DEPARTING FROM A HORIZONTAL SURFACE 
prevented from wiping the wall clear of water (see discussion in section (I-6), it could be assumed that in nucleate boiling the radius of the cavity could be substituted for the radius $r_{0}$ of the orifice in Equation III-1. This would enable us to compute the spectrum of diameters of cleparting bubbles in terms of the spectrum of cavity diameters. We cannot test this assumption because no experimental data are available. This can be done somewhat indirectly for the experiments of Fritz and Ende (24) and of Zmola (42), who reported the values of $D_{\mathrm{d}}$. No data was given however for cavity. dimensions $D_{c}=2 r_{0}$. The analysis in Section II-5 indicated a possible relation between the diameter of the cavity $D_{c}$ and the thickness of the superheated liquid film $x_{s}$. If we substitute $x_{s} / 2$ from Equation II-26 for $r_{0}$ in Equation III- 1 , it follows that

$$
D_{d}=\left[\frac{6 \sigma}{g\left(f_{L}-p_{L}\right)} \frac{k \Delta T}{q}\right]^{1 / 3}
$$

On Table III-1 the experimental data of Zmola are tabulated together with the computed value of $D_{d}$. 
TABLE III-1

Comparison of Equation III-6

with Experimental Data of Zmola

$\begin{array}{cccc}\begin{array}{c}\Delta T \\ \text { OF }\end{array} & \begin{array}{c}\mathrm{q} \\ \text { Btu }\end{array} & \begin{array}{c}D_{\mathrm{d}} \\ \text { in }\end{array} & \begin{array}{c}D_{\mathrm{d}} \\ \text { (Equation III-6) } \\ \text { in }\end{array} \\ & & & \\ 9.4 & 9600 & 30 \times 10^{-3} & 32 \times 10^{-3} \\ 9.4 & 9600 & 31 \times 10^{-3} & 32 \times 10^{-3} \\ 9.4 & 9600 & 34 \times 10^{-3} & 32 \times 10^{-3}\end{array}$

The same is done on Table III-2 for the experiments of Fritz and Ende and of Jakob and Linke.

\section{TABLE III-2}

Comparison of Equation III-6 with Experimental Data of Fritz and Ende and of Jakob and Linke

Fritz and Ende

$\Delta_{O C}^{T} \frac{\mathrm{Kcal}}{\mathrm{hr} \mathrm{m}^{2}}$

$\begin{array}{llll}9 & 19000 & 5.42 \times 10^{-1} \\ 9 & 19000 & 4.04 \times 10^{-1} \\ 9 & 19000 & 4.64 \times 10^{-1}\end{array}$

$D_{d}$
Jakob \& Linke Equation

$D_{\mathrm{d}}$

$\mathrm{cm}$

(average value)

$$
\begin{array}{ll}
2.81 \times 10^{-1} & 2.15 \times 10^{-1} \\
& 2.15 \times 10^{-1} \\
& 2.15 \times 10^{-1}
\end{array}
$$

It is seen that the computed values are of the same order as the measured one. In view of the possibilities which a relation of this kind could open, it appears that further experimental investigations are warranted. 
III-4. On the Similarity Between Hucleate Boiling and Flow of a Gas Bubbling Through a Perforated Surface.

We shall discuss in this section the similarity which exists between nucleate boiling and a gas bubbling through a perforated or porous surface. From this similarity and from some phenomena which occur in fluidized bubble-bed apparatus, the mechanism leading to the critical heat flux in nucleate boiling will become apparent .

The problem of a gas bubbling through a porous surface has received much attention recently. The reader is referred to the papers by Verschoor (68), Siemes $(60,61)$, Kolbel and Siemes (69), Siemes and Borchers (70) and Houghton, McLean and Ritchie (71) for detalled discussion and additional references. It was suggested already by Kolbel and Siemes that experiments performed with bubble-beds, i.e., with a gas flowing through a porous plate, may be used to help our understanding of nucleate boiling. Such experimental studies of two-phase flow phenomena conducted with perforated and porous surfaces were recently reported by Petrik (72) and by Wallis and Griffith (73). 
We shall first examine the relation between the gas flow rate and the pressure drop across the porous surface; the discussion is based on the studies reported in References 61,70 and 71 . Talke a capillary of a given radius $r_{0}$, the pressure difference which is required for the formation of a bubble is given by Equation I-1. Thus the problem of initiating the bubbling process in nucleate boiling and from a porous plate are identical.

Consider now the effect of increasing the gas flow rate. At low gas flow rates only few of the largest pores are bubbling. As the gas flow increasea the pressure difference increases and an increasing number of smaller pores become active. Vershoor noted that in addition to these new pores coming into action the first ones are yielding larger bubbles. Smaller pores can be brought into action also by reducing the surface tension of the liquid. The porosity of the surface determines the slope of the curve. This can be seen on Figure III-2, which is reproduced from Reference 70 .

The reader should compare now the experimental results listed above to the discussion, given in Section I-4, of the effect of surface roughness upon the relation between the heat flux density and superheat 


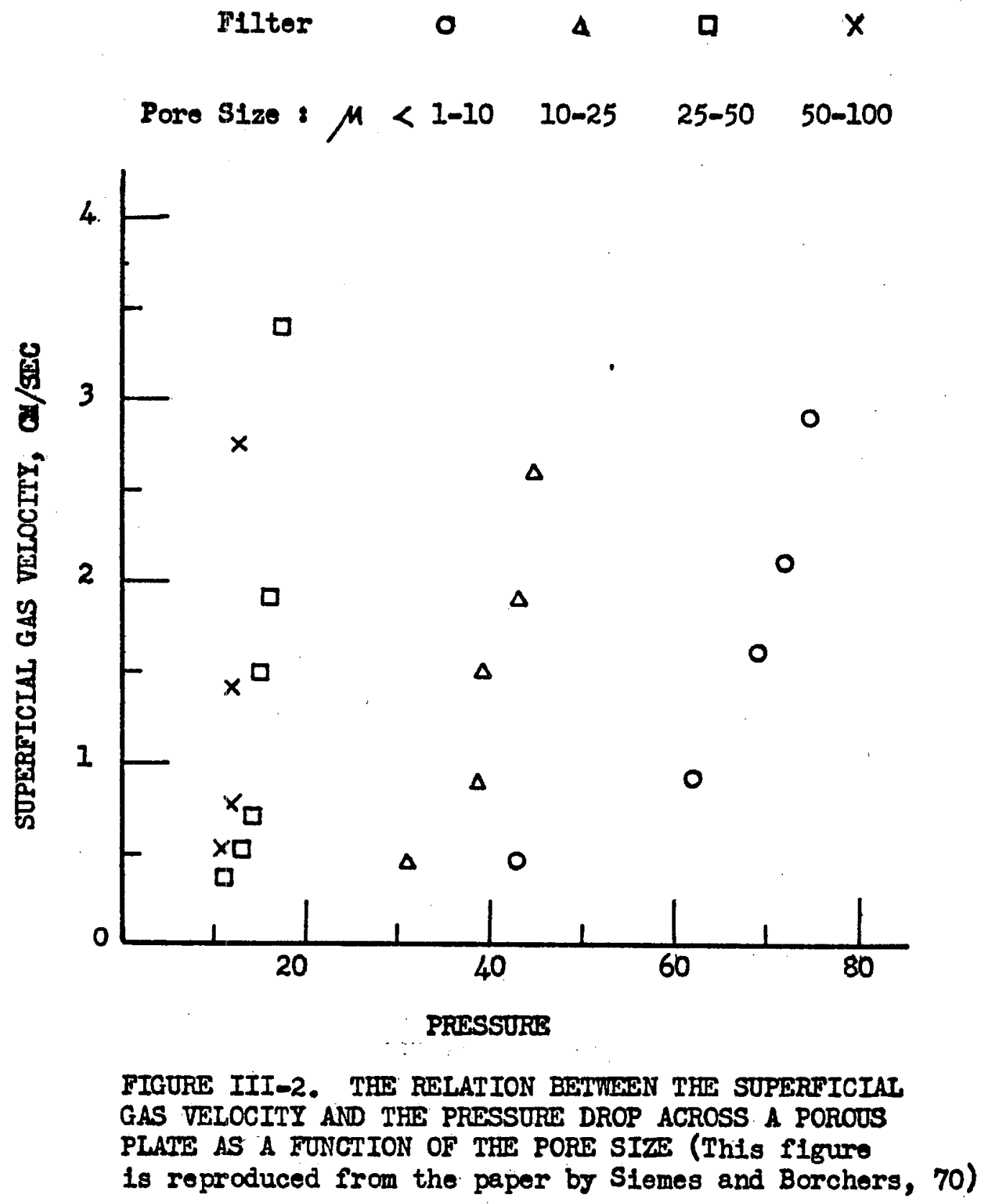


temperature difference $T_{W}-T_{S}$, in nucleate boiling. If, as it was suggested in Section III-1, "gas flow rate" is substituted for "heat flux density", both descriptions are alike. Note that through ClausiusClapeyron's equation the superheat temperature difference can be expressed in terms of a pressure drop.

We shall now examine some hydrodynamic characteristics of fluidized bubble-beds. For additional information the reader is referred to the excellent study of siemes. The flow process of gas bubbling. through a porous plate is characterized by two distinct flow regimes. At low gas flow rates a so-called "laminar" regime exists, whereas a "turbulent" regime is associated with high gas flow rates. At low gas flow rates few pores are active. Bubbles of constant volume rise with a constant velocity wi thout interacting. At these low gas flow rates an increase of flow rate results mostly in an increase of the number of acting pores, i.e., of the bubble population. At still higher rates both the population and the bubble volumes increase and bubbles start interacting. This process continues until, at a critical gas flow rate, the flow changes to a turbulent process. Houghton and co-workers (71) observed that this change in the flow regimes is associated with bubble coalescence. If the gas flow rate is still further increased large plugs of gas are formed. 
The distinct difference between these two flow regimes is shown on Figure 2 in Reference 69. It was noted that hydrodymamically, nucleate boiling is similar to the process of a gas bubbling from a porous or perforated plate. Indeed, if Figure 2 from Reference 69 Is compared to the photographs of nucleate boiling which were published by Westwater and co-workers $(1,25)$, no difference can be seen. The "laminar" regime corresponds to nucleate boiling at low heat transfer rates, whereas the "turbulent" regime corresponds to the region close to the critical heat fluse. It appears thus that the critical heat flux is characterized by a change in the flow regime. We shall explore this statement further.

III-5. The Phenomenon of "Flooding"

The change of flow regimes and, consequently, of the operating conditions is not a characteristic of fluidized bubble-beds only, but is a phenomenon which occurs in the performance of other apparatus. It has been recognized that the liquid and gas throughput in packed columns is subject to an upper limit above which the column ceases to operate satisfactorily as a countercurrent device. This upper limit is designated either as the "loading" or the "flooding" point. For a given apparatug "flooding" is determined by both gas and liquid rates. At any given gas rate there is a definite liquid 
rate above which a packed column will flood. Similarly, at any given liquid rate there is a definite gas rate above which the column will flood.

The mechanism of "flooding" in a sieve-tray distillation tower was recently described by McAllister, McGinnis and Plank (74). Among other effects, "flooding" resulted from a complete lifting of the liquid from the tray floor and subsequent carry-over of all the liquid on the tray as entrainment.

The generality of the "flooding" phenomenon was best discussed by Elgin and Weiss (75) and we shall quote now their description of the mechanism.

"It is believed desirable to emphasize here the generality of flooding and to visualize it as a requisite consequence in any apparatus in which one fluid passes discontinuously through an immiscible second one. The flooding of the packed tower is a special case of this general viewpoint. Bubble-cap, spray, bubble, or film type gas-liquid or liquid-liquid towers exhibit flooding in essentially the same manner. Preliminary visual laboratory studies of the flooding of such equipment indicate no funciamental or radical difference in the operative mechenism producing flooding. A difference in the visual behavior of such equipment arises from differences in the phase initially discontinuous. The 
behavior of an evaporator or vaporizer operated at an excessive heat rate for its diameter or a tube containing a column of water into which air is bubbled at an increasing rate is believed to be an evidence of the same basic phenomenon.

"The general character of flooding is visualized as follows: As pointed out the holdup of any discontinuous phase passing through another, whether the latter is in motion or not, necessarily increases with its rate of flow since its linear velocity in the continuous is less than proportional to the rate at which it enters. That its holdup must eventually approach the free volume or cross section of the apparatus and result in a tendency for it to become continuous and displace the phase Initially continuous is obvious. The continuous phase, If in countercurrent motion, retards the passage of the discontinuous through frictional drag to a greater or less extent. An increase in the holdup of the latter follows. This in turn results in a corresponding increase of holdup. Since the countercurrent velocities are mutually interdependent, the effect is multiplied at a rapidly accelerating pace with further increase in volume of either phase fed to the apparatus and must eventually culminate in flooding. When the flooding point is reached, either of two things may happen. Depending 
upon the rate of passage of the continuous phase if made discontinuous in the other and $i$ ts corresponding holdup at the relative volume flow rates at which flooding has occurred, either the discontinuous phase is reversed, and the only evident change is a transition say, from a spray to a bubble device; or if the flow conditions are such that the corresponding holdup of each would be sufficient to fill the available volume, a competition results and either is discharged more or less violently from one or both ends of the apparatus. Both conditions occur in packed tower or a spray type liquid-liquid tower. Only the latter situation is observed in a film type or a bubble type gas-liquid tower. The first may be regarded as flooding with respect to Iiquid, the second to gas."

We can interpret now the critical heat flux as the region where vapor and liquid compete for the free volume. It is a hydrodynamic phenomenon like other "flooding" phenomena. We may expect, therefore, that it will occur when the velocity of the vapor phase reaches a critical value. Like in the experiments of McAllister and Plank (74) the liquid will be pushed away from the surface and the whole system will oscillate at some characteristic Erequency. In Chapter VI we shall determine the critical velocity of the vapor phase, and this fre- 
quency. In the next section we shall discuss the heretofore proposed correlations of the critical heat flux.

III-6. Previous Emplrical Correlations of the Critical

Heat Flux

The observation of Elgin and Weiss which was quoted in the preceding section is, to the knowledge of the writer, the first reference in the literature which interpreted the critical heat flux as a hydrodynamic phenomenon. The first correlation of experimental data of the critical heat transfer rates which used the concept of "flooding" was reported by Bonilla and Perry (75).

The correlation of experimental data for column flooding are plotted in terms of two dimensionless groups

$$
\begin{aligned}
& \pi_{1}=\frac{\rho_{g} u_{q}^{2}}{\rho_{L} 2 g m} \\
& \pi_{2}=\frac{u_{g}}{u_{L}}
\end{aligned}
$$


where $U_{g}, U_{L}$, and $\rho_{g}, \rho_{L}$ are the superficial velocities and densities of the gas and liquid phase respectively; whereas $m$ and $g$ are the hydraulic radius and the acceleration due to gravity. To apply the above dimensionless groups to the critical heat flux, Bonilla and Perry substituted the group $q / L \rho_{v}$ for $U_{g}$, and

$\rho_{I} / \rho_{V}$ for $U_{g} / U_{L}$. The hydraulic radius was expressed in terms of the diameter $D_{\mathrm{d}}$ given by Equation I-7. The correlation was expressed thus in terms of

$$
\begin{aligned}
& \pi_{3}=\frac{\left(q / L \rho_{V}\right)^{2} \rho_{V}}{\rho_{L} 2 g \frac{1}{4}\left[\frac{\sigma}{g\left(\rho_{L}-\rho_{V}\right)}\right]^{1 / 2}} \\
& \pi_{4}=\frac{\rho_{L}}{\rho_{V}}
\end{aligned}
$$

The experimental values of the critical heat flux $q$, for boiling binary liquid mixtures, were inserted in Equation III-8, and the data was plotted on a $\log -\log$ scale using $\pi_{3}$ and $\pi_{4}$ as coordinates. From the results Bonilla and Perry concluded, "The points do not fall too far from the flooding line." However, in their subsequent publications this approach to the critical heat flux was not pursued further. 
The view that the critical heat flux marks a transition from one regime to another was adopted by Kutateladze $(78,77)$, Borishanslkii $(80,79)$, Sterman (56), and by Rohsenow and Griffith ( $8 \phi)$. The analysis of Kutateladze will be discussed in greater detail because he was responsible for the investigation and interpretation of the critical heat flux as a hydrodynamic phenomenon.

Kutateladze noted $(78,77)$, "At heat transfer rates close to the critical heat flux, the amount of vapor forming on the surface is so large that it is Impossible to consider separate nucleating centers and separate bubbles. Consequently, in this case the meaning and the concept of the frequency of bubble generation is lost. . the essential feature of the theory of the phenomenon can be derived if one assumes that the crisis in the boiling process is purely a hydrodynamic phenomenon: the destruction of stability of two-phase flow existing close to the heating surface." According to this view the change from nucleate to transitional boiling occurs when the velocity in the vapor phase reaches a critical value. Starting from the non-linear Euler equation of motion and the energy equation he derived by dimensional analysis, the following equation for the critical heat flux in pool boiling of 
liquids at saturation:

$$
\frac{q}{L\left(\rho_{v}\right)^{h_{L}\left[\sigma g\left(\rho_{L}-\rho_{v}\right)\right]^{1 / 4}}}=K=\text { const. }
$$

III-9

The constant $\mathrm{K}$ was determined from experiments and its value was found to be

$$
K=0.16
$$

The same equation, using dimensional analysis but a different thought model, was re-derived by sterman (56).

It is interesting to note here that the dimensionless group $K$ defined by Equation III-9 is equal to the square root of the dimensionless group $\Pi_{3}$ defined by Equation III-8.

In two recent papers Borishanskif $(80,79)$ extended Kutateladze's analysis to include the effect of viscosity. In the discussion he pointed out several interesting aspects of nucleate boiling close to the critical heat $f l u x$. These comments are worth repeating here. According to Borishanskii the continuous existence of steady heat transfer rates in the neighborhood of the critical nucleate heat flux leads to the conclusion that there exists a direct steady movement of liquid toward the heating surface and of vapor away from it. Because 
of the density difference between the two phases, Borishanskii reasoned that a large part of a crosssectional area close to the heating surface is occupied by vapor rather than by liquid. Therefore, near the heating surface one can consider a two-phase boundary layer whose thicleness is of the order of a disengaged bubble. This two-phase boundary region may be visualized as consisting of liquid streams flowing toward the surface and surrounded by vapor. The shapes of the fllaments of liquid as they flow towards the wall are not well defined because of the inherent randomness in the bubble dynamics and coalescence in the nucleate regime.

At low nucleate boiling heat transfer rates the discrete phase appears as a vapor bubble surrounded by a mass of liquid; whereas close to the critical heat flux it is, rather, a liquid stream filament bounded by a group of bubbles. The change from nucleate to trans1tional boiling occurs when the steady flow of the liquid toward the wall is disrupted, 1.e., when at critical velocity of the vapor phase the liquid streams are destroyed. Borishanskii noted further that: "This problem seems malogous from a theoretical point of view to the disturbance of steady flow of a liquid stream in gas which moves coaxially with it. The 
solution of the problem leads to a relationship between the increment of the osclilation and the wave length. Further analysis of the equation for the limiting case of stable flow leads to the conclusion that the critical boiling point corresponds to the establishment of a definite geometrical structure of the two-phase boundary layer." Borishanskii, therefore, considered that the phenomenon was to be explained by analyzing the stability of a liquid jet surrounded by a moving, coaxial, vapor phase. From the equation which determines the amplitude of the wave and from the energy equation he established, by dimensional analysis, two similarity criteria: $\mathrm{K}$ given by Equation III-9 as previously found by Kutateladze, and $N$, given by

$$
N=\frac{\rho_{L} \sigma^{3 / 2}}{\mu^{2}\left[g\left(\rho_{L}-e_{V}\right)\right]^{1 / 2}}
$$

By plotting $K$ versus $\mathrm{N}$ he found the following approximate relation:

$$
K=0.13+4 N^{-0,4}
$$


In order to establish the above correlation, 117 experimental data points were used. The experimental data represent the following liquid-solid combination: Water boiling on a graphite surface (77), ethanol, benzene, n-heptane, and n-pentane boiling on a chromium plated surface (82), ethanol (83) and water (84) bolling from a nichrome surface. The viscosity of the liquid appears in Equation III-12 only in the additive correction factor N. Inasmuch as the deviation from a horizontal line is small it can be seen from Figure III-3, that the effect of the viscosity is also sinall.

Good agreement with experimental data was achieved, also, by the correlation proposed by Rohsenow and Griffith (8) :

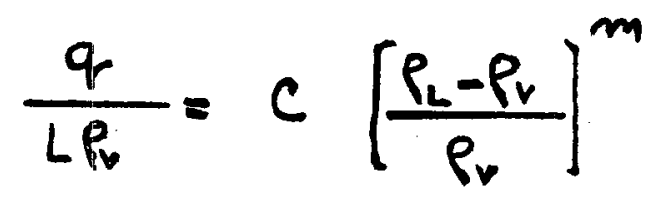

III-13

The value of the constant c. $=143$ f.p.h., which has dimensions of a velocity, and the exponent $m=0.6$ were determined from experimental data by plotting $q / L P_{v}$ versus the buoyancy term $\rho_{L}-\rho_{v} / \rho_{v}$.

Equations III-9 and III-13 are renarkable for three reasons. We note first that they do not relate heat transfer to a temperature difference. Also, they 


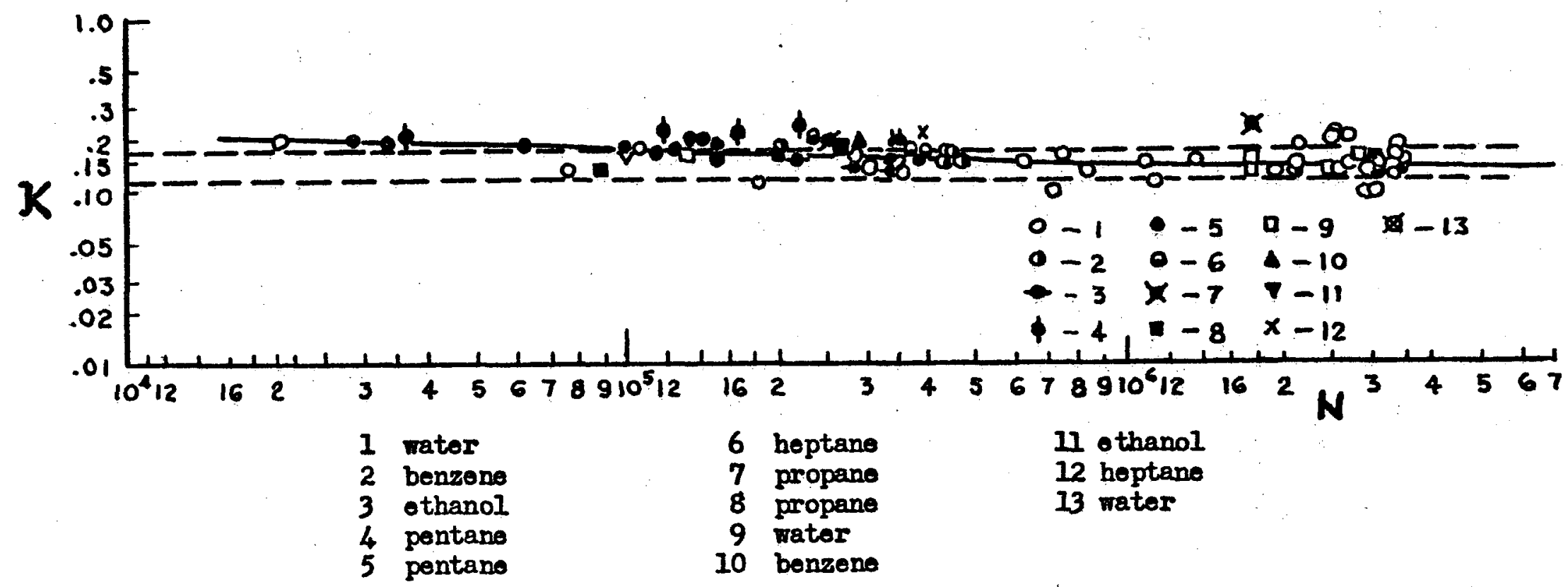

FIGURE III-3. CORBEIATION OF DATA FOR VARIOUS LIQOIDS AT THE CRITICAL HEAT FLUX DENSITY IN POOL BOIITNG.

This figure is reproduced from the report by Borishanskif $(79,80)$. The solid line (Equation III-12) 1s his best fit curro to 117 data points. The two dashed lines represent the upper and lower bounds on $\mathrm{K}$ (Equation VI-13) derived by the considerations in the paper. 
do not depend upon the thernal and transport properties of the liquid. And, we note their striking simplicity. These characteristics of Equations III-9 and III-13 are surprising when an interpretation of the phenomenon is sought in terms of the conventional convective heat transfer concepts where the thermal and transport properties of the liquid are of paramount importance. It is not surprising, however, if the critical heat flux is interpreted as a hydrodynamic phenomenon of "flooding" which occurs at a critical velocity of the vapor phase. This statement is confirmed when Equation III-13 is compared to the equation which gives the critical, 1.e., the limiting, vapor velocity at which a bubble-cap column floods (BS).

$$
U_{G}=K\left[\frac{\rho_{L}-\rho_{v}}{\rho_{v}}\right]^{1 / 2}
$$

where $U_{c}$ is the maximum permissible velocity, in feet per second, based on the area of the bubbling section of the plate and $K$ is an empirical constant. 
III-7. Nucleate Boiling and the Region of the Critical Heat F1ure

- In Chapter I we have reviewed experimental results of nucleate boiling. In the preceding sections of this chapter, nucleate boiling and the process of a gas bubbling through a liquid were compared and two similarities were analyzed. We have considered first the similarity which exists between a gas bubbling through an orifice and bubbles growing from a nucleus in bolling. Following this, we have considered the similarity which exists between nucleate boiling and a gas bubbling through a perforated surface.

By malking use of the first similarity and of some known facts concerning the frequency of bubble emission from an orifice, an equation (Equation III-4 or III-5) was derived for the product $D_{d}$.f in nucleate boiling. It is possible thus, from Equation III-4, to estimate the maximum frequency of emission of simple bubbles in nucleate boiling. The first similarity indicated also the possibility of expressing the diameter $D_{\mathrm{d}}$ of a simple bubble departing from the heated surface in terms of the radius of $r_{0}$ of the nucleating cavity. The second similarity, i.e., the similarity which exists between nucleate boiling and the process of a gas forced to bubble through a perforated surface, 
Indicated the basic nature of the phenomenon which Induces the change from nucleate to transitional boiling. It was seen that the critical heat flux is a hydrodynamic phenomenon. It was concluded also that extsting correlations (cf. Equation III-13) can be understood only If the critical heat flus is interpreted as a "flooding" phenomenon.

In this section we shall integrate the discussion given in Chapter I with the results presented in the - preceding sections of this chapter. We shall attempt thus to form a composite picture of nucleate pool bolling from a horizontal aurface.

Consider the effect of increasing the heat flux density on the process of boiling. At low heat flux densities nucleate boiling talkes place in patches. In these patches of nucleate bolling "simple" bubbles are generated (see Figure III-4.a). From a given nucleating center bubbles depart with a regular frequency and with a constant diameter. The product of the diameter and the frequency can be approximated by Equation III-4. To a distribution of nucleating cavities of different dimensions there corresponds probably a distribution of departing bubbles having different diameters and different frequencies of emission. The velocity of bubble rise in the vicinity of the surface can be approximated 


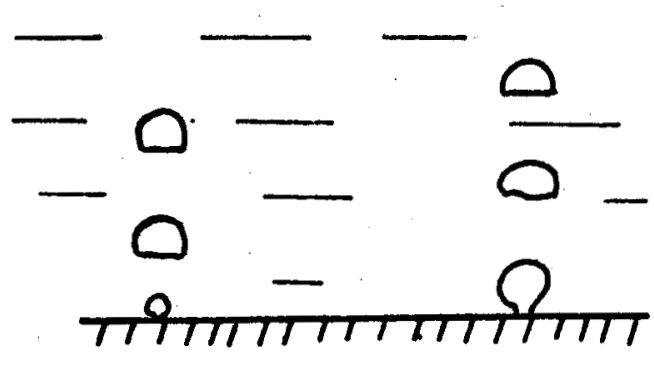

a.

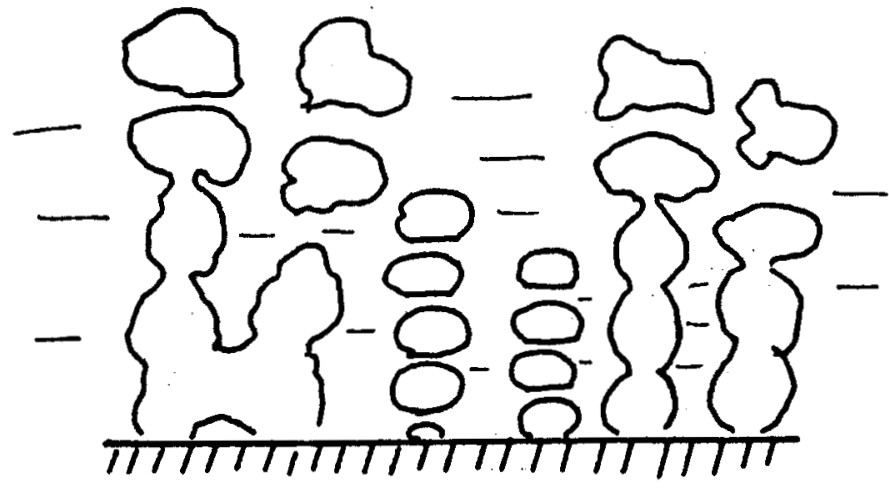

d.
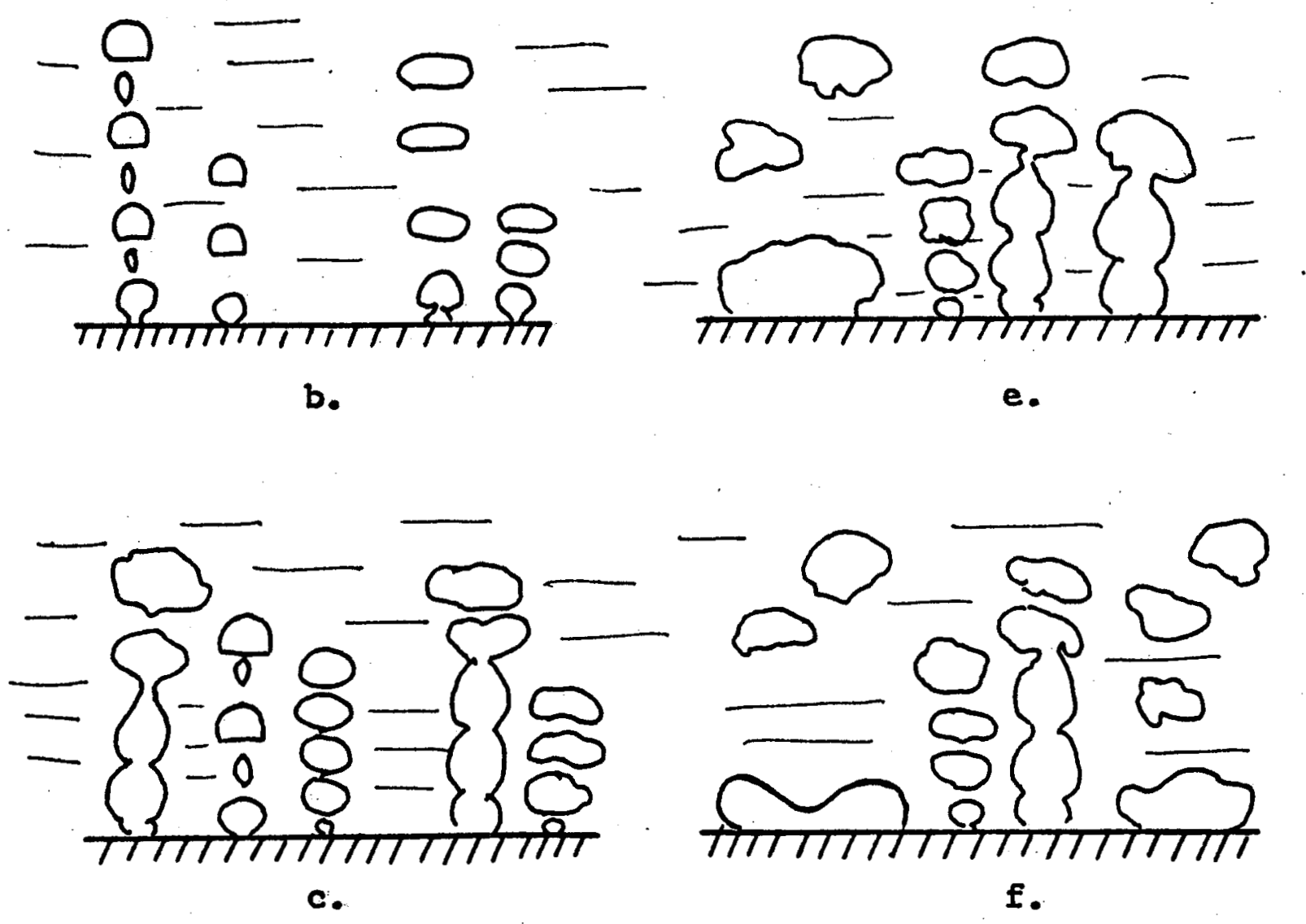

FIgure III-4. Schematic Representation of the Process of Nucleate Boiling 
by Equation III-2. As bubbles grow and depart the liquid is agitated. At regular time intervals in the vicinity of a nucleating center colder liquid is brought in contact with the heating surface, thus resulting in high local heat transfer rates. The process of bubble generation and, consequently, the process of local heat transfer are intermittent. The sum of such high local heat transfer rates results in an average total heat flux density which is higher than in a non-boiling system.

As the heat flux density is increased the patches of nucleate boiling spread, 1.e., the bubble population increases. In addition to the new active nucleating centers the first active ones are generating "multiple" bubbles (see Figure III-4.b). The process of spreading of patches of nucleate boiling continues with an increase of the heat flux density until the whole ourface is covered with active sites.

The bubble population continues to increase with Increasing heat flux densities. The frequency of bubble emission increases until bubbles follow each other, le., the contact time is almost zero. For a given bubble diameter the maximum frequency can be estimated from Equation III-4. Under these conditions coalescence of bubbles takes place. Consequently, from some nucleating 
centers continuous vapor columns, 1.e., vapor jets, are generated. At some distance from the surface these vapor jets breals up Into large bubbles (see Flgure III4.c). It is probable that further increase of the heat flux density results mainly in increasing the number of these continuous vapor columns, although the bubble population wL11 keep Increasing al 80 .

The vapor velocity in these jets increases with increasing heat flux density. The process continues until at sorre critical velocity of the vapor two neighboring jets start interacting, 1.e., become unstable. The IIquid stream between the two jets is interrupted, and the steady flow of the liquid toward the surface ceases (see Figure III-4.d). Using the expression of Elgin and Welos the vapor and the liquid start competing for the free volume and "flooding" starts.

For a horfzontal aurface the interaction of the fets occirs at a randon place. Thus, at random location on the surface a large patch of vapor is formed (see Tigure III-4.e). In order to distinguish the patches of nucleate bolling which appear at low heat flux densities from the large patches of vapor which appear In the region close to the critical heat flux, we shall refer to the latter oncs as patches of transitional boiling. Such a patch insulates the surface locally. 
Consequently, for a constant heat flux density the temperature of the surface increases. This causes the rate of evaporation to increase and results in higher vapor velocities in other jets. These, in turn, become unstable, start interferring with each other and patches of transitional boiling appear at a compounding rate. Using, again, the expression of Elgin and Weiss the effect of the instability is multiplied at a rapidly accelerating pace. Thus in the region of the critical heat flux a small increase of the heat flux density results in a large increase of the surface temperature. Consequently, In the $q=\Delta T$ plane there exists a plateau across which boiling changes from the nucleate to the transitional boiling regime.

The patches of transitfonal boiling are hydrodynamically unstable; this problem will be analyzed in the chapter which follows. In collapsing, i.e., when the vapor-1iquid interface of a patch approaches the heated surface, large rates of evaporation occur and the interface is pushed violently back. The process is similar to explosions or bursts of vapor. Similarly to the "flooding" of sieve-trays, in the region of the critical heat flux, the liquid is pushed away from the surface and the whole system oscillates at some characteristic frequency. 
Once the patches of transitional boiling cover the whole surface, transitfonal bolling sets in. The problem of transitional bolling is taken up in the chapters which follow. It will be seen that a well defined geometry is the characteristic of transitional bolling. Therefore, an analytical solution of the problem can be attained $(86,87,88)$. In nucleate bolling the flow configuration is not well defined. As noted by Borishanskil, "the form of the Iiquid streams differs considerably since $1 t$ is determined by the not well regulated order of the combination of vapor bubbles." It was observed in Reference 88, that given a field equation and no geometrical data, Kutateladze and Borishanskil found it necessary to use dimensional analysis, which is the best that can be done under the circumstances and which reduces the problem to the determination of an empirical constant.

III-8. Nucleate Boiling Heat Transfer - Methods of Analysis

In this closing section of the chapter on hydrodynamic aspects of nucleate boiling we shall discuss briefly two methods of analyzing the process of heat transfer in nucleate boiling. Correlations of experimental data which are based on finding empirical relations among dimensionless groups will not be discussed 
here. This approach to the problem as well as the correlations which have been proposed are discussed in Reference 88 .

It was discussed in the preceding sections that heat transfer rates in nucleate boiling are the result of local effects which are created by bubbles growing and departing from the heated surface. Consequently, an analysis must consider both the effect of bubble population and the mechaniam of vapor removal.

The problem can be analyzed either by considering the transport of enthalpy across an Imaginary boundary located in the liquid, or by considering the mechanism of energy transfer at the heating surface. A complete analysis cannot be carried out at present because both methods require information on the bubble population; this information is not available yet. In what follows we shall only outline these two methods of analysis and cite the contributions and suggestions which were advanced by various researchers.

The transport of enthalpy which was considered by Sterman (56) and by Treschov (20) is an energy balance: the enthalpy transported by one bubble, multiplied by the frequency of emission and by the number of bubbles per unit area, is equated to the heat flux density, thus. 


$$
q=\frac{4 \pi}{3} \bar{R}^{3}\left[c \rho_{L}\left(\overline{T_{w}-T_{L}}\right)+L \rho_{V}\right] \vec{f} \frac{n}{A}
$$

where the bar refers to mean values.

The second method of analysis was proposed by Kruzhilin (54, 55) and by Rannie (47); they considered the heat transfer process at the heating surface. It was noted that, because of the growth and departure of a bubble, colder liquid is periodically brought in contact with the heating surface. Thus in the vicinity of a nucleating center large temperature gradients are created periodically; conduction is increased and becomes the dominant heat transfer process. The analysis is formulated $(54,55)$ by considering the amount of energy which is transferred from the heated surface $(x=0)$ during a contact time $t_{c}$, across an area element $F$. This area $F$ is the segment of the total heated surface which is influenced by the action of one nucleating center, i.e., it is the "influence domain" of one bubble. The amount of energy $Q^{\prime}$, which is transferred by the action of one nucleating center, becomes thus

$$
Q^{\prime}=\left.\int_{t_{\alpha}}^{t_{d}+t_{c}} \int_{F} k \frac{\partial T}{\partial x}\right|_{x=0} d t d F
$$


The total amount of energy Q, which is transferred from the surface, is obtained by multiplying Equation III-15 by the total number of nucleating centers.

In orcler to make use of Equation III-IS it is necessary to evelliate the gradient $(\partial T / \partial x)_{x=0}$. This temperature gradient will be determined, as in similar transient conduction problems, by the coupling of the thermal fields which exist in the solid and in the liquid. It will depend thus upon the thermal properties of both the solid and the liquid. The importance of the thermal characteristics of the solid on nucleate boiling heat transfer were discussed by Rohsenow, Saberslky and Snyder (47). The observation made by Zysina-Molozhen, which was quoted in Section I-4, only adds supporting evidence. It was discussed by Griffith (44) that when the temperature of the heated surface remains approximately constant during the process of intermittent heat transfer, the temperature distribution in the liquid during the contact time $t_{c}$, can be approximated by the solution of Equation II-2. The instantaneous temperature gradient is given then by Equation II-4.

When the energy equation for the liquid adjacent to the heating surface can be approximated by Equation II-2, the problem of intermittent heat transfer in nucleate boiling becomes similar to the system which is 
described by the "discontinuous film model." This model was developed and used by Highbie (89), Dankwerts (90) and Hanratty (91) to describe the exchange of mass, momentum and energy at a boundary. According to this theory, masses of fluid can be visualized as moving to and from the wall causing a continual change of the fluid in contact with the wall. When a mass of fluid come $s$ in contact with the solid, its surface layer Immediately attains the temperature of the wall. It remains in contact with the wall for a period of time $t_{c}$, during which energy is transferred from the wall to the liquid by conduction. The problem is similar to the heating of a block of metal. After the contact time $t_{c}$, the mass of fluid is replaced and the process is repeated.

The problem is formulated by considering the Instantaneous temperature gradient, in this instance it is given by Equation II-4. If all fluid masses had the same contact time $t_{c}$, the average, i.e., the measured heat flux density would be

$$
q=\frac{1}{t_{c}} \int_{0}^{t_{c}} \frac{k\left(T_{w}-T_{s}\right)}{\sqrt{\pi a t}} d t={\frac{2\left(T_{w}-T_{s}\right)}{\sqrt{\text { Tat }}}}^{\text {III-I7 }}
$$


However, different liquid masses will have different contact times. The effect of the variations is taken Into account by considering the probability $\phi\left(t_{c}\right) d t_{c}$ that a fluid mass has a contact time between $t_{c}$ and $t+d t_{c}$. The average heat flux is obtained by integrating over all variations, thus

$$
q=\int_{0}^{\infty} \phi\left(t_{c}\right) \frac{2 x\left(T_{\omega}-T_{s}\right)}{\sqrt{\pi a t_{c}}} d t_{c}
$$

It is seen from this brief discussion that the system which is described by the "discontinuous film model" resembles the process of intermittent heat transfer which takes place in nucleate boiling. An investigation along this line will be given at a later date. Here we note that this approach can be readily applied to transitional boiling. It will be seen in the chapters which follow that, in contrast to nucleate pool bolling where the frequency of bubble emission and therefore the contact times are given by a distribution curve, in transitional boiling to a given heat flux density there corresponds one frequency. Thus, in transitional boiling Equation III-18 is reduced to Equation III-17 which permits an analytical solution of the problem $(87,88)$. 
HYDRODYNAMIC ASPECTS OF TRANSITIONAL BOILING

The region of the critical heat flux was discussed in the preceding chapter. It was observed that when two vaporjets start interferring with each other the flow of Iiquid toward the surface is interrupted and a patch of vapor, i.e., a patch of transitional boiling, is formed. The region of the critical heat flux is characterized thus by the presence of both nucleate and transitional boiling. When patches of transitional boiling cover the whole surface transient boiling sets in. We shall analyze in this chapter the hydrodynamic aspects of transitional boiling. The first analytical investigation of the characteristics of transitional boiling was reported in Reference 86 ; the problem was investigated further in Reference 88.

In transitional boiling the heating surface is separated from the liquid phase by an unstable irregular film of vapor. The process is hydrodynanically unstable, because the acceleration is directed from the less dense to the more dense redium. An understanding of the phenomenon requires therefore an understanding of the rech- 
anism of hydrodynamic instability, a topic which is taken up in the section which follows.

\section{IV-1. Stability of a Plane Interface - Mathematical Formulation}

The problem of hydrodynamic stability of a plane interface has received much attention. The reader is referred to the treatise by Lamb (50) and in particular to the recent monograph by Birkhoff and Zarantonello (92) for a detafled discission of the problem. In what follows we shall summarize the results which are pertinent to the problem under consideration. The dis cussion Is based on studies which were reported by Taylor (93), Lewis (94), Allred and Blount (95), Bellman and Pennington (96) and by Birkhoff $(97,98)$.

When two liquids of different densities and having a comon interface are accelerated in a direction perpendicular to the boundary, any small Irregularity of the interface will tend to change in shape. The interface is stable, i.e., the irregularities will tend to smooth out in time, if the acceleration is directed from the denser to the lighter fluid. The interface is unstable, 1.e., the irregularities of the interface will grow with time, when the acceleration is directed from the lighter to the heavier medium. A familiar example of this phenomenon is a glass of water turned upside down. The 
air-water interface instead of remaining plane as it falls will tend to form long liquid spikes which subsequently disintegrate into drops. An instability of this kind is referred to as "Taylor instability" because, as pointed out by Birkhoff and Zarantonello, "the first clear formulation of this principle was due to Sir Geoffrey Taylor (93), who also inspired its experimental confirmation by Lewis." (94)

The problem was formulated mathematically by Kelvin (Reference 50, Page 458) using potential theory. Consider a plane interface at $Y=0$ separating the vapor in $Y<0$ and the liquid in $Y>0$. Let an infinitesimal perturbation of this interface be given by

$$
\eta=H_{0} e^{i(\omega t-m x)}
$$

IV-1

Under the usual perturbation approximation the frequency equation of the interface becomes (Reference 50, Page. 459, Equation 2)

$$
\omega^{2}=\frac{\sigma m^{3}}{p_{L}+P_{V}}-\frac{g\left(P_{L}-\rho_{V}\right) m}{p_{L}+P_{V}}
$$


The condition of stability is that $\omega$ be real; when $\omega$ becomes imaginary the disturbance $\eta$, given by Equation IV-I, will grow exponentially with time. The wave length can be expressed in terms of the wave number $m$, thus,

$$
\lambda=\frac{2 \pi}{m}
$$

Equation IV-2 Indicates that the disturbances of the interface can be stable or unstable according to whether the wave length is shorter or longer than a critical value. This critical value is obtained by equating Equation IV-2 to zero, thus

$$
\Lambda_{01}=2 \pi\left[\frac{\sigma}{g\left(\rho_{L}-\rho_{V}\right)}\right]^{1 / 2}
$$

Equations IV-I and IV-2 indicate the existence of a continuum of unstable perturbations, each of which has its own growth rate. Because of the exponential nature of the instability the wave length which maximizes - $\omega 2$ will be the "most dangerous", i.e., it will result in a disturbance which has the most rapid growth. Differentiating $\omega 2$ with respect to the wave numer $m$, the "most dangerous" wave length then becomes (96): 


$$
\Lambda_{02}=2 \pi\left[\frac{3 \sigma}{g\left(e_{L}-e_{V}\right)}\right]^{1 / 2} \quad \text { IV } \quad
$$

Substituting Equation IV-5 into Equation IV-2, the maximum value of $-\omega 2$ becomes

$$
\omega^{2}=-\frac{2}{3} \frac{g\left(\rho_{L}-\rho_{V}\right)}{\rho_{L}+\rho_{v}}\left[\frac{g\left(\rho_{L}-\rho_{V}\right)}{3 \sigma}\right]^{1 / 2} \quad I V-6
$$

The most unstable disturbance is obtained from Equation IV -1 and IV- 6 , thus

$$
\eta_{m}=H_{0} e^{\beta t} e^{i m x}
$$

where

$$
\beta=\left[\frac{2 g\left(\rho_{L}-\rho_{V}\right)}{3\left(\rho_{L}+\rho_{V}\right)}\right]^{1 / 2}\left[\frac{g\left(\rho_{L}-\rho_{V}\right)}{3 \sigma}\right]^{1 / 4}
$$

IV -8

The comparison of these theoretical results with experimental data is discussed in the section which follows. 
IV-2. Stability of a Plane Interface - Experimental

\section{Resulto}

We shall first note some phenomena which can be interpreted in terms of Taylor instability. It was observed by Beliman and Pennington (96) that, "This phenomenon explains the hanging of water drops on the underside of a horizontal surface, such as a ceiling. Such a droplet is undergoing an upward acceleration of $980 \mathrm{~cm} / \mathrm{sec}^{2}$ and will tend to drip because of Taylor instability unless its effective wave length is about $\lambda_{01}=1.73 \mathrm{~cm}$. Droplets of larger diameter will tend to drip, while smaller ones will tend to hang." Another evidence is cited by Lamb (50) and Milne-Thompson (99) who point to experiments "in which water is retained by atmospheric pressure in an inverted tumbler whose mouth is closed by a gauze of sufficiently fine meshes." The mesh size should not exceed $\Lambda_{01} / 2$.

It was noted in Section III-I that Davidson and Amick (64) reported "... stable stationary bubbles could not be found at an orifice having a diameter of $0.79 \mathrm{~cm}$, but stable bubbles formed at an orifice of $0.64 \mathrm{~cm} . "$ The reason for this becomes apparent when Taylor instability is considered. For water the critical wave length $\Lambda_{01}$ is $1.73 \mathrm{~cm}$; because of probable fluctuation at the orifice or in the liquid, the orifice diameter 
of $0.79 \mathrm{~cm}$ was too close to the value of $\Lambda_{01} / 2$ for the stability to be maintained.

The occurrence of capillary waves in stable film boiling has been reported and described by Bromley (100) and by Westwater and Santangelo (1). In a recent paper Chang (101) observed that in stable film boiling the bubble spacings computed from Equation IV-4 are in agreement with experimental results reported by Westwater and Santangelo (1).

The analytical prediction of Taylor and of Bellman and Pennington were verified by the experiments reported by Lewis (94) and by Allred and Blount (95). These experiments were conducted to determine the growth of sinusoidal perturbations with wave length $\Lambda$ at a liquid-gas interface, accelerated at $a=30 \mathrm{~g}-75 . \mathrm{g}$ over a distance of $5 \lambda-15 \lambda$. Lewis (94) concluded that "the instability is made up of the following stages:

1) "An exponential increase in amplitude as given by the first order theory until the amplitude is about $0.4 \lambda$.

2) "A transition stage during which the amplitude increases from $0.4 \lambda$ to $0.75 \lambda$ and the surface disturbance changes to the form of round-ended columns of air penetrating into the 1iquid, which forms narror upstanding 
columns in the interstices.

3) "A final stage of penetration through the liquid of the columns at a uniform velocity proportional to $\sqrt{a-g}$."

Two additional stages to be expected are discussed by Birlchoff (97); these are:

4) "A stage in which the boundaries of the air columns will deform irregularly under the influence of Helmholtz instability and the growth of vorticity, until

5) "the mixing zone separating the two fluids is turbulent, and must be analyzed (like turbulence) by statistical methods."

For detailed information concerning Taylor instability the reader is referred to this excellent report by Birlihoff.

The initial exponential growth rate as predicted by Taylor's theory was verified further by the experiments of Allred and Blount (95). They observed also that during the final stage of deformation (Stage 3 ), the interface consists of spikes of heavy liquid extending into the light fluid, and of rounded regions which may be thought of as bubbles of lighter fluid rising into heavier fluid. The spacing of these spikes, i.e., the effective "wave length" was found to be in good agreement with the "most 
dangerous" wave length given by Equation IV-5. It is important to emphasize here that this equation was derived from two-dimensional considerations.

Lewis (94) reported that the growth rates during the asymptotic stage can be predicted from an equation, derived by Davies and Taylor (102), for the rise of a large bubble:

$$
u=\frac{2}{3}[r g]^{1 / 2}
$$

were $r$ is the radius of curvature of the bubble vertex. Similar conclusions for the asymptotic growth were reported by Allred and Blount (Reference 95, Page 11)*. The asymptotic growth rate of Taylor instability was investigated also theoretically in the recent papers by Garabedian (103) and by Birkhoff and Carter (104).

In this and the preceding section we have briefly sumarized results of theoretical and experimental investigations of Taylor instability. It will be seen in the sections which follow that transitional boiling is closely related to this instability.

* See also Reference 97, Page 16 and Page 31. 
IV-3. Westwater and Santangelo's Description of

Transitional Boiling

The only quantitative, experimental investigation of transitional boiling in the literature was reported by Westwater and Santangelo (1). It is fortunate to have as a guide for theoretical analysis the results of these and other outstanding experiments reported by Professor Westwater and his co-workers. Westwater and Santangelo give the following description of transition boiling.

'Wost prior worlers have failed to realize that this boiling is entirely different from both nucleate boiling and film boiling. No active nuclei exist. In fact, no liquid-solid contact exists either. The tube is completely blanketed by a film of vapor, but the film is not smooth nor stable. The film is irregular and in violent motion.

"Vapor is formed by sudden bursts at random locations along the film. Liquid rushes in toward the hot tube, but before the two can touch, a miniature explosion of vapor occurs and the liquid is thrust back violently. The nevly formed slug of vapor finally ruptures, and the surrounding liquid again surges toward the tube. The process is repeated indefinitely. 
"One observer of these high speed motion pictures has expressed an opinion that occasional liquid-solid contact does occur during transition boiling. If so, these contacts are rare and exceedingly short. The present writers do not believe there is a real contact.

"The frequency of the vapor burst is surprisingly high. For an over-all $\mathrm{T}_{\mathrm{w}}-\mathrm{T}_{\mathrm{g}}$ of $133^{\circ} \mathrm{F}$ ( and of $h=164 \mathrm{Btu} / \mathrm{hr} \mathrm{ft}^{2}{ }^{\circ} \mathrm{F}$ ) each inch of the photographed side of the tube exhibited 34 bursts per second. The burst occurs so suddenly and unexpectedly that even in slow motion they resemble explosions.

"If the temperature is increased still further, the blanket becomes thicker and more stable. ... The increase of vapor thickness results in a better damping of the explosive burst until finally a thick rather stable film results and no more bursts occur. This is the beginning of film boiling."

The minimum heat flux, i.e., point $D$ on Figure I is described by Westwater and Santangelo as follows:

"As vapor gathers at the top of the tube, a rodlike mass forms along the top of the entire length of tube. At first the rod is smooth and rather uniform in thiclmess, although faint movements occur constantly. As the gas rod increases in volume, peales and valleys form until the upper boundary becomes sinusoidal in shape. 
The entire rod ruptures, between all the nol es, and a horizontal row of bubbles rise side by side. After the rupture, the whole process is repeated. One cycle requires 0.06 second when the over-all temperature difference is $184^{\circ} \mathrm{F}$.

"A second gas rod ruptures at points directly under the centers of the last released individual bubbles. This means that the even-numbered rows of bubbles are displaced sideways by a half space from the odd-numbered ones. Viewed from the side the bubbles are similar to marchers arranged in triangular spacing rather than the conventional square spacing."

In the section which follows we shall relate these observations to the discussion which was presented in the preceding section. We shall thus interpret transitional boiling in terms of Taylor instability.

IV-4. The Hydrodynamic Instability of Transitional Boiling

Consider a vapor patch in transitional boiling from a horizontal surface (see Firure IV-1,a). The liquid vapor interface is hyclrodynamically unstable because the acceleration is directed from the vapor to the liquid. It can be expected that, because of agitation, the interface has random initial perturbations, 


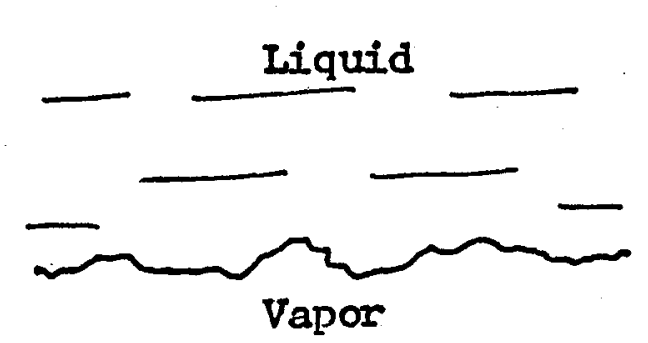

गाTाTाभTाTाTा

a.

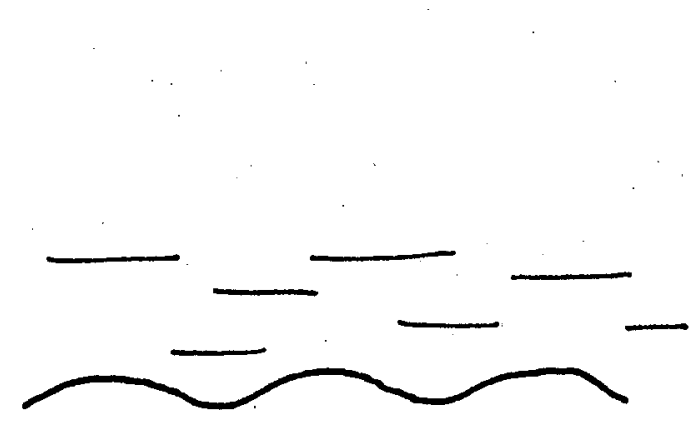

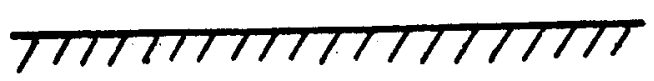

b.

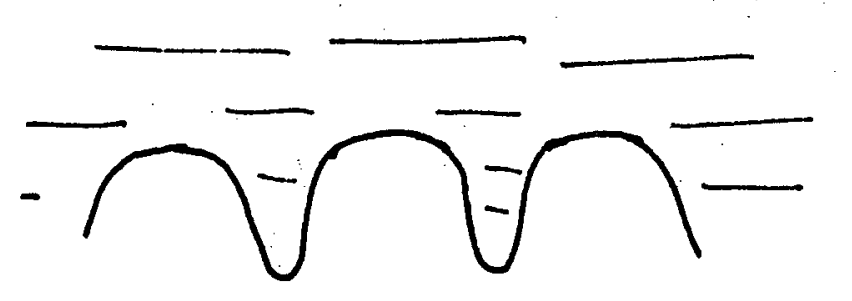

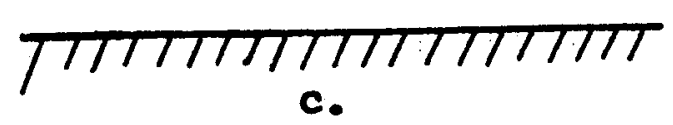

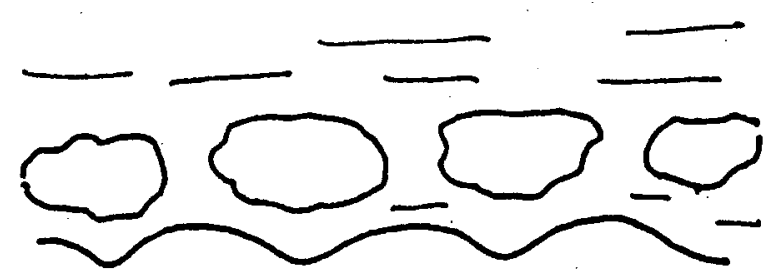

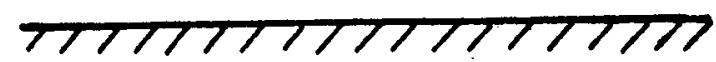

d.
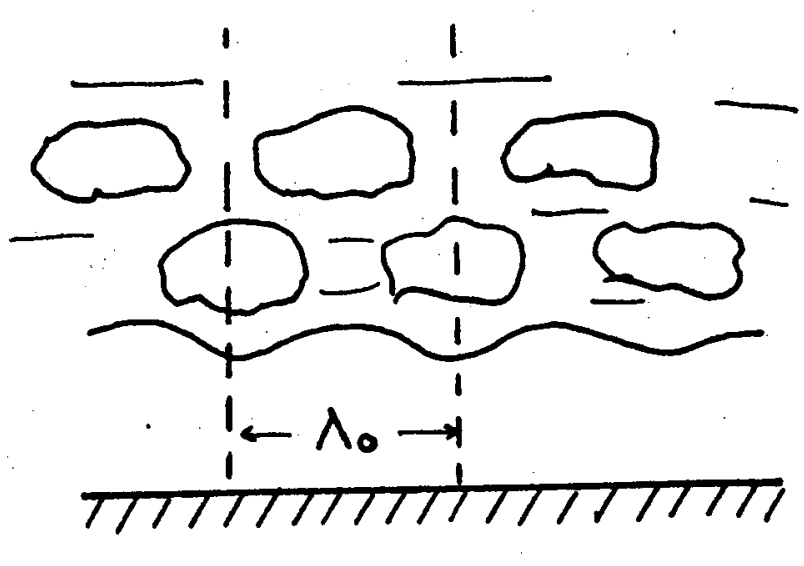

e.

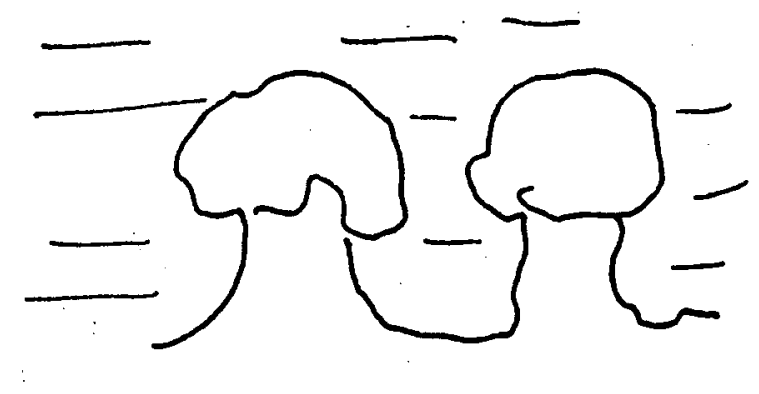

गाTाTाग7ाা

f.

Figure IV-1 Schematic Representation of the Process of Transitional Boiling 
distributed over a spectrum of wave lengths.

It was seen in Section IV-2 that the growth rates and wave lengths which are obtained by two-dimensional considerations are in good agreement with experimental results. It appears, therefore, that the essential characteristics of the physical process are well described by two-dimensional equations. In view of the above agreement we shall, in what follows, make use of this two-dimensional thought model. The analysis can be easily extended to three dimensions. Indeed, the three-dimensional frequency equation was published by Masavell (Reference 50, Page 461).

It was seen in Section IV-1 that for a twodimensional system all perturbations with wave lengths longer than the critical one, i.e., than $\lambda_{\text {ol }}$, are unstable. For an interface which has random initial perturbations it can be expected that, because of the initial exponential growth, wave lengths near the "most dangerous" one, 1.e., near $\lambda_{02}$, will be the first to achieve finite observable amplitude. In line with the experiments of Lewis and of Allred and Blount, the interface will consist of spikes of liquid and of rounded regions similar to cylindrical bubbles which rise into the liquid. Therefore, as a consequence of Taylor instability a definite geometrical configuration in 
transitional boiling can be expected (see Figure IV-1.b). This chape of the interface is shown by the photograph on Figure 10.4 in Reference 105. For a two-dimensional system this geometry should be characterized by disturbances with wave lengths in the spectrum

$$
\lambda_{\text {o1 }} \leq \lambda_{0} \leq \lambda_{02} \quad \text { IV-10 }
$$

In contrast to nucleate boiling, where the disturbances of the superheated liquid film originate at randomly distributed nucleating centers, in transitional boiling the disturbances occur away from the surface and are selected by the properties of the fluid field.

In their downward fall the spikes approach the heated surface and rapid evaporation occurs. As liquid evaporates from the spilces the vapor flows in the region between two spiles. It was noted that this region resembles rising bubbles (see Figure IV-1.c). The same form of the interface is shom by the photographs on Figure 7, Reference 1, and on Figures 3.6, 3.7, 3.8, and 3.9 in Reference 95. These and other photographs are reproduced in Reference 88 . Thus in transitional boiling because of Taylor instability a release of bubbles at regular intervals can be expected. 
As a row of bubbles is released an unstable interface is formed again. Because of the downward flow of the liquid a spike will be formed now underneath the released bubble and the process is renewed (see Figure IV-1.d). The successive rows of bubbles will appear displaced therefore by half a wave length (see Figure IV-1.e). To quote Westwater and Santangelo, "the bubbles are similar to marchers arranged in triangular spacing." This spacing is shown by photographs on Figure 8, Reference 1, and on Figure 14.3, Reference 106. The alternate interchange of position between the spiles and the rounded cylindrical bubbles bears similarity to the phenomenon known as Faraday's crispations which is discussed by Rayleigh (107). We shall quote his description of the phenomenon. When a horiaontal plate on wich liquid is spread is set to vibrate, "the motion of the liquid interface consists of two sets of stationary vibrations superposed, the ridges and furrows of the two sets being perpendicular to one another and usually parallel to the edges of the plate - . At one moment the ridges form a set of parallel and equidistant lines, the interval being $\lambda$. Midway between these are the lines which represent, at that monent, the position of the furrows. After a lapse of a $1 / 4$ period, the surface is flat; after another $1 / 4$ 
period, the ridges and furrows are again at their maximum development but the positions are exchanged." In the region of transitional boiling close to the critical heat flux, the rates of evaporation are high. Consequently, the release of bubbles will resemble small explosions or bursts of vapor (see Figure IV-1.f). A similar form of the interface is shown by photographs on Figures 4 and 5 in Reference 1 and Figure 5.6 in Reference 95. The mushrooming of the interface during the growth of Taylor instability was observed in the experiments by Allred and Blount who noted: "It seems reasonable to ascribe this effect to the Helmholtz instability, in view of the fact that the necessary velocities for the production of Helmholtz instability are present." A similar effect can be expected to take place in transitional boiling at high heat flus densities when large rates of evaporation occur, i.c., when the velocities in the vapor phase are large. This aspect of the problem will be analyzed in Chapter VI.

It was observed that the geometry of the interface in transitional boiling is determined by the properties of the fluid field. Inasmuch as the factors which influence the "most dangerous" wave length will remain constant at a given pressure, it can be expected that, in transition boiling, changes in heat transfer rates 
are associated with changes in the frequency of bubble. release, i.e., of the vapor bursts. The critical heat fluxs and the minimum heat flux correspond, therefore, to the maximum and the minimum frequencles of the system. At a given heat flux the process of transitional bolling can continue indefinitely; it is therefore thermally stable. However, it is hydrodynamically unstable; Indeed, it is this instablifty which is the cause of the phenomenon.

In this chapter we have qualitatively analyzed the hydrodynamic aspects of transitional bolilng. It was seen that experimental results of transitional boiling can be understood and interpreted in terms of Taylor instability. We shall summarize now the results of this chapter.

1) As a consequence of Taylor instability a definite geometrical configuration can be expected in transitional boiling. For a two-dimensional system this geometry is characterized by disturbances with wave length in the spectrum.

2) As a consequence of the exponential character of the instabllity it can be expected that the interface takes the form of spikes of heavy liquid moving downward and of rounded 
reglons of lighter fluid moving upward.

3) As a consequence of the definite geometrical configuration a release of bubbles from the interface at regular intervals can be expected. For large evaporation rates the release of a bubble will appear as a burst.

4) Because of the release of vapor and of Taylor Instability the process exhibits, also, a periodicity in time. The phenomenon is hydrodynamically unstable but thermally stable.

5) Inasmuch as the factors which influence the geometry remain invariant, it can be expected that, in transitfonal boiling, changes in heat transfer rates are associated with changes in frequency only. The critical heat flux and the minimum heat flux correspond, therefore, to the maximum and the minimum allowable frequencies of the system. In the chapters which follow, the problem will be formulated mathematically and the results quantitatively compared to experimental data. 


\section{CHAPTER V}

THE MINIMUM HEAT FLUX DENSITY IN TRANSITIONAL BOILINO FROM A HORIZONTAL SURFACE

The geometrical regularity and the periodicity of the process of transitional boiling was discussed in the preceding chapter. It was noted that inasmuch as the geometry should remain invariant, changes of heat transfer rates should be assoclated only with changes of the frequency of the system. The phenomenon thus bears a similarity to a release of bubbles from a set of orifices of fixed geometry but with a variable frequency. Although, at first, this similarity appears to be rather tenuous the agreement of the detail and the gross characteristics of the idealized system with experimental data seems to support it. The problem is, therefore, to determine the geometry of "orifices" and the maximum and minimum frequency of the releases. According to the hypothesis, these frequencies should correspond to the maximum (critical) and to the minimu heat transfer rates in transitional bolling from a horizontal surface. In this chapter we shall analyze the minimum heat flux density; the critical heat flux 
density is talcen up in Chapter VI.

V-1. Minimum Heat Flux Density - Formulation and

Solution of the Problem

In order to formulate the problem a simplifying

idealization must be made concerning the form of the vapor slugs which are released from the vapor liquid interface. Westwater and Santangelo observed (see Section IV-3) that the disturbed vapor IIquid Interface breaks at the nodes. It will be assumed therefore in what follows that the vapor slugs can be approximated by spheres of radius

$$
R=\frac{\Lambda_{0}}{4}
$$

For the two dimensional thought model* it follows from Equation IV-10 that the diameter is given within the Iimits

$$
\pi\left[\frac{\sigma}{g\left(\rho_{L}-\rho_{V}\right.}\right]^{V_{2}} \leq D \leq \pi\left[\frac{3 \sigma}{g\left(\rho_{L}-\rho_{V}\right)}\right]^{1 / 2}
$$

* See Section IV-4 for the discussion concerning the two-dimensional approximation. 
The mass of vapor associated with one vapor slug is

$$
G=e_{v} \frac{4 \pi}{3}\left(\frac{\Lambda_{0}}{4}\right)^{3} \quad v-3
$$

Denoting the frequency of bubble release by $f$, the number of bublbles released per unit time is

$$
n=\frac{a}{\Lambda_{0}} \cdot \frac{b}{\lambda_{0}} f
$$

where $a \cdot b$ is the area of interest. The vapor flow rate per unit area is obtained from Equation $V-4$ and $V-3$, thus

$$
\dot{M}=\rho_{V} \frac{\pi}{48} \Lambda_{0} f
$$

The heat transfer is obtained from an energy balance. In transitional boiling of liquids at saturation the only energy requirements of the system is the energy needed to generate the vapor flux density given by Equation V-5. Therefore the heat transfer rate from a horizontal surface in transitional boiling of liquids at saturation is 


$$
q=L \rho_{r} \frac{\pi}{48} \Lambda_{0} f
$$

At a constant pressure the geometry does not change; Equation $V-6$ can be written as

$$
q=(\text { constant }) \cdot f
$$

The problem now is to determine the frequency of bubble release $f$,for the minimm heat flux density. It was discussed in the preceding chapter that at the minimum heat flux the rates of evaporation are low, consequently in this region small velocities in the vapor phase can be expected. In the absence of dynamic effects of the vapor phase, the rate of penetration of the rounded vapor regions into the liquid (see Figure IV-1.c) cannot be faster than that which is predicted by Taylor instability. In other words, at the minimum heat flux in transitional boiling from a horizontal surface, the frequency of release of bubbles from the vapor-liquid interface cannot be slower than that which would be expected by considering the effect of Taylor instability only. The relation between the frequency of bubble release $f$, and the rate of penetration of the interface $d \eta / d t$, then becomes 


$$
F=\frac{2}{\lambda_{0}} \frac{d \eta}{d t}
$$

The above relation implies that bubbles follow one another very closely in a strip of width $\lambda_{0}$, which extends from the heating surface into the liquid (see Figure IV-1.e). The photograph on Figure 14-3 in Reference 106 confirms the validity of this approximation.

To make use of Equation $\mathrm{V}-8$ we have to determine the value $d \eta / d t$. It was discussed in Sections IV-I and IV-2, that the growth of Taylor instability passes through several stages. During the initial stage the growth rate can be predicted from the linearized theory, whereas during the final stage the rate of growth can be approximated by Equation IV-9. We shall express the frequency in terms of both growth rates.

Consider the "most dangerous" wave length $\lambda_{02}$. If the amplitude $\mathrm{H}_{0}$, of the disturbance $\eta$, was known then Equation IV-7 could be used to estimate the time which is required for the disturbance to reach a value of $\eta_{m}=\Lambda_{02} / 2$. This method was used by Birlchoff (Reference 97, Page 41) in analyzing the penetration of a disturbance. However, in boiling the values of $\mathrm{H}_{0}$ are not reported. In order to estimate the initial rate 
of growth we shall make use of the experimental obserration of Lewls (94) that the amplitude increases at an exponential rate until it reaches a value of $\eta=0.4 \Lambda$ (see Section IV-2). From Equation IV-7 the rate of growth during the initial stage is given by:

$$
\frac{d \eta}{d t}=\beta \eta_{m}
$$

During the exponential growth the disturbance $\eta \mathrm{m}$ increases from an infinitesimally small value to approximately $0,4 \Lambda_{02}$. The average velocity corresponding to this average disturbance is

$$
\frac{\overline{d \eta}}{d t}=\beta \frac{0.4}{2} \Lambda_{02}
$$

Substituting Equations $V-10$ and IV-8 into Equation V-8, the estimated frequency of bubble release becomes

$$
f=0.4 \beta=0.4\left[\frac{2}{3} \frac{g\left(\rho_{L}-\rho_{V}\right)}{\rho_{L}+\rho_{V}}\right]^{1 / 2}\left[\frac{g\left(\rho_{L}-\rho_{V}\right)}{3 \sigma}\right]^{1 / 4}
$$


From Equations $V-6$ and $V-11$ the estimated minimum heat flux density is then given by

$$
\left.q=L e_{v} \frac{\pi}{48} 2 \pi\left[\frac{3 \sigma}{g\left(l_{L}-e_{v}\right.}\right]^{1 / 2} 0.4\left[\frac{2}{3} \frac{g\left(l_{L}-e_{v}\right)^{1 / 2}}{l_{L}+l_{V}}\right]^{1 / 2\left(l_{L}-e_{v}\right)}\right]_{v-12}^{1 / 4}
$$

or

$$
q=L e_{v} \frac{\pi}{24}\left(\frac{\pi 0.4 \sqrt{2}}{(3)^{V_{4}}}\right)\left[\frac{\sigma g\left(\rho_{L}-e_{V}\right)}{\left(\rho_{L}+P_{V}\right)^{2}}\right]^{1 / 4}
$$

We shall now use the growth rate of the final stage, i.e., Equation IV-9 to estimate the frequency and the heat flux density. Including the effect of buoyancy in Equation IV-7 and substituting Equation V-1 for the radius of curvature $r_{0}$, Equation IV-9 becomes

$$
u=\frac{2}{3}\left[\frac{\Lambda_{0}}{4} \frac{g\left(P_{L}-e_{v}\right)}{e_{L}}\right]^{1 / 2}
$$


144

The frequency is obtained from Equation $v-14$ and $V-8$, thus,

$$
f=\frac{2}{3}\left[\frac{q\left(p_{L}-e_{V}\right)}{e_{L}} \frac{1}{\Lambda_{0}}\right]^{1 / 2}
$$

Because of the spectrum of unstable disturbances, the wave length in the above expression can be determined between the limits given by Equation IV -10, 1.e., by Equations IV -4 and IV -5. It follows from Equation V-15, IV -4 and IV -5 that the frequency can be determined within the limits:

$$
\begin{aligned}
& \frac{2}{3}\left[\frac{g\left(f_{-}-f_{1}\right)}{e_{L}} \frac{1}{2 \pi}\left(\frac{g\left(e_{6}-f_{v}\right)}{3 \sigma}\right)^{1 / 2}\right]^{1 / 2} \leqslant f \leqslant \\
& \text { V-16 } \\
& f \leqslant \frac{2}{3}\left[\frac{q\left(p_{L}-e_{V}\right)}{p_{L}} \frac{1}{2 \pi}\left(\frac{g\left(p_{L}-e_{V}\right)}{\sigma}\right)^{1 / 2}\right]^{1 / 2}
\end{aligned}
$$

Consequently the period can be estimated within the range

$$
\begin{aligned}
\frac{3}{2}\left[\frac{\rho_{L}}{g\left(\rho_{L}-\rho_{V}\right)} \cdot 2 \pi\left(\frac{\sigma}{g\left(\rho_{L}-\rho_{V}\right)}\right)^{1 / 2}\right]^{1 / 2} \leqslant \tau \leqslant \\
\leqslant \frac{3}{2}\left[\frac{\rho_{L}}{g\left(\rho_{L}-\rho_{V}\right)} 2 \pi\left(\frac{3 \sigma}{g\left(\rho_{L}-\rho_{V}\right)}\right)^{1 / 2}\right]^{1 / 2}
\end{aligned}
$$

$\mathrm{V}-17$ 
Equations $V-4$ and $V-16$ give the range of the minimum heat flux, thus

$$
\begin{aligned}
& L e_{v} \frac{\pi}{48} \Lambda_{00} \frac{2}{3}\left[\frac{g\left(e_{L}-e_{v}\right)}{e_{L}} \frac{1}{\Lambda_{01}}\right]^{1 / 2} \leqslant q \leqslant \\
& q \leq L \rho_{V} \frac{\pi}{48} \Lambda_{02} \frac{2}{3}\left[\frac{g\left(\rho_{L}-\rho_{V}\right)}{\rho_{L}} \frac{1}{\Lambda_{02}}\right]^{1 / 2} \quad v-18
\end{aligned}
$$

Hence from Equations IV -4 and IV-5 it follows that

$$
\begin{aligned}
L \rho_{v} \frac{\pi}{24} & \frac{\sqrt{2 \pi}}{3}\left[\frac{\sigma g\left(\rho_{L}-\rho_{V}\right)}{e_{L}^{2}}\right]^{1 / 4} \leqslant q \\
q & \leqslant L \rho_{v} \frac{\pi}{24} \frac{\sqrt{2 \pi}}{3}(3)^{1 / 4}\left[\frac{\sigma g\left(\rho_{L}-\rho_{V}\right)}{\rho_{L}^{2}}\right]^{1 / 4}
\end{aligned}
$$

By comparing Equation V-19 to V-13 and Equation V-16 to $V-11$, it is seen that they exhibit identical dependence upon physical properties. These equations vary only in the values of the numerical constants; the variations being of the order of unity. We note also that the above equations are of the same form as those which have been reported in Reference 86 and 88 . The latter ones were derived by considering the similarity between the oscillations of the interface in transitional boiling and the Faraday's crispations, a similarity 
which was noted in Section IV-4.

In the section which follows we shall compare theoretical predictions to experimental data.

V-2. The Minimum Heat Flux Density - Comparison of Analytical with Experimental Resulta

In the following, the theoretical predictions are compared with experimental data for bolling methanol at atmospheric pressure reported by Westwater and Sentangelo (1).

The Diameter of Bubbles at the Minimum Heat Flux Analysis. Experiment (inches) (Inches)

Equation V-2

$$
0.2 \leq D \leq 0.345
$$$$
0.2 \leq D \leq 0.36
$$

The Period at the Minimum Heat Flux

$$
\text { Analysis }
$$

(seconds)
Experiment (seconds)

Equation V-11

$\tau=1 / f=0.052$

0.06

$$
0.048 \leqslant \tau \leqslant 0.063
$$


The Minimum Heat Flux in Trangition Boiling

$$
\text { Analjgis }
$$

(Btu/hr $f t^{2}$ )
Experiment

(Btu/hr $f t^{2}$ )

Equation V-13

Equation V-19 $q=8740$

$5500 \leqslant q \leqslant 7100$

It appears from the above comparisong that this almple idealized aystem exhibits the detailed and general features of the observed phenomenon. The spectrum of bubble diameters as determined from experIments tends to support the original assumption concerning the spectrum of unstable disturbances and the approximation related to the form of the vapor 8lugs. The frequency of bubble release and the minimum heat flux density determined from the theory are in close agreement with experimental data. Thus, the description and statements concerning transition bolling which were shown, in the preceding chapter, to be in qualitative agreement are also in quantitative agreement with experimental data.

It should be noted that this agreement was achieved by two dimensional considerations; further experiments should indicate whether a three dimensional modification Is necessary. Further experiments in transitional 
bolling should be performed in order to Investigate, in detail, the growth of a diaturbance during both the exponential stage and the final stage of penetration. We shall proceed with an analysis of the critical heat flux. 
CHAPTER VI

\section{THE CRITICAL HEAT FLUX IN BOILING \\ FROM A HORIZONTAL SURFACE}

The region of the critical heat flux was discussed in section III-7. It was seen that the phenomenon occurs at some critical velocity in the vapor phase when the vapor jets start interferring with each other. It was seen also that because of the spreading of the patches of transitional boiling the region of the critical heat flux corresponds to a plateau in the $q-\Delta T$ plane. This region is clearly shown by the experimental results of Perkins and Westwater (25). Consequently, an analysis of the critical heat fluxs can be undertaken at either end of this plateau. Because the flow configuration in nucleate boiling is not well defined, an analysis of the critical heat flux performed by considering nucleate boiling is reduced to dimensional analysis. It was seen In the preceding chapter that, because of Taylor instability, a definite geometrical configuration can be expected in transitional boiling. We shall make use of this defined geometry and analyze, therefore, the critical heat flux by considering transitional boiling. 
VI-1. The Critical Heat Flux - Formulation and Solution of the Problem

It was discussed in the preceding chapter that in transitional boiling at high flux densities the release of bubbles appears like vapor explosions. As the interface mushes toward the surface rapid evaporation starts, the interface is pushed violently back, and the vapor $1_{8}$ released in the form of explosive jets (see FIgure IV-I.f). The process is repeated indefinitely and, as in "flooding", the whole system oscillates at some characteristlc frequency. In Section IV-4 the observation of Allred and Blount was quoted which related the mushrooming form of the Interface (see Figure IV-1.f) to Helmholtz Instab1I1ty. A similar effect can be expected to take place at the critical heat flux when, because of the large rates of evaporation, the velocity of the vapor phase is large. Thus, at the critical heat flux the dynamic effect of the vapor phase upon the motion of the Interface become important. This is in contrast to the minimum heat flux. where this dynamic effect is negligible. Whereas the minimum heat flux is characterized by Taylor instability only, the critical heat flux is characterized by the combined effects of. Taylor and Helmholtz instabilities. In order to formulate the problem it is necessary to determine in what was these instabilities manifest 
themselves and interact.

It was noted that the phenomenon of transitional bolling, because of Taylor instability, bears similarity to a release of bubbles with variable frequency from a set of regularly spaced orifices of fixed geometry. In accordance with the hypothesis, at the critical heat flux the frequency reaches a maximum and the release resembles explosive vapor jets. In view of Taylor instabllity we are lead, therefore, to consider vapor columns of width, $\Lambda_{\%} / 2$, spaced $\Lambda_{0}$ units apart. In view of Helmholtz Instability we have to Investigate the stability of such a jet. In accordance with the previous analysis the stability of a two-dimensional system is investigated again.

Consider a coordinate system in which the direction $y$ is parallel to the surface and $z$ is perpendicular to the surface. Let the plane $y=0$ denote an interface between the vapor leaving the heated surface and the fluid rushing toward 1t. For a vortex sheet which oscillates under the influence of surface tension, the propagation equation of a small disturbance is given by Lamb (50) and Milne-Thomson (99):

$$
c^{2}=\frac{\sigma m}{\rho_{L}+\rho_{b}}-\frac{\rho_{L} \rho_{v}}{\left(\rho_{L}+\rho_{v}\right)^{2}}\left(u_{v}+u_{L}\right)^{2}
$$


The condition of stablifty is that $\mathrm{C}$ shall be real.

The velocity in the liquid phase is obtained from the continuity

$$
e_{V} u_{V}=\rho_{L} u_{L}
$$

VI-2

Substituting $U_{I}$ from Equation VI-2 Into Equation VI-I the critical velocity in the vapor phase is then obtalned, thus

$$
u_{v}=\left[\frac{\sigma m}{P_{v}}\right]^{1 / 2}\left[\frac{P_{L}}{P_{L}+P_{v}}\right]^{1 / 2}
$$

It is noted that if, In a three-dimensional problem, the continuity equation was corrected for the areas occupied by the vapor and the liquid, the right hand side of Equation VI-3 would be multiplled by the factor $P_{L}(16-\pi)+P_{V} \pi / R_{L}(16-\pi)$. The value of this factor is unity and it is omitted therefore.

We have to determine now the value of the wave number $m$ in Equation VI-3. Rayleigh (107) has examined the stability of a circular gas jet in a liquid. For a disturbance with axial symmetry the instability occurs only for disturbances whose wave length is longer than the circumference of the jet. Thus, the critical wave length is given by 


\section{$\lambda=2 \pi R$}

$$
\text { VI-4 }
$$

where $R$ is the radius of the jet. If the jet is to breale into spheres, it can be expected that the wave lengths will be of the same order of magnitude axially as circumferentially. Expressing therefore the wave number $m$ in Equation VI-3 in terms of the wave length $\Lambda$, given by Equation VI-4, the critical vapor velocity then becomes

$$
u_{v}=\left[\frac{\sigma}{\rho_{v} R}\right]^{1 / 2}\left[\frac{\rho_{L}}{\rho_{L}+e_{v}}\right]^{1 / 2}
$$

In Appendix I, the above equation is derived by considering the stability of a circular jet. It was assumed that the radius $R$, of the vapor column was given by $\Lambda_{0} / 2$; substituting this value in Equation VI-5 it follows that

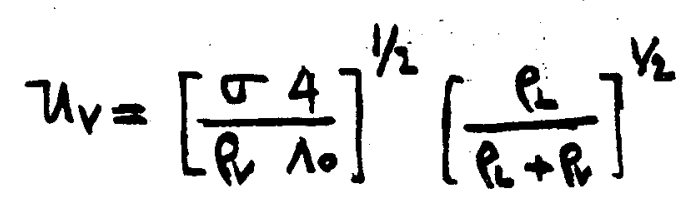

The mass flow rate associated with one vapor column is: 
154

$$
G=\rho_{v} \pi\left(\frac{\Lambda_{0}}{4}\right)^{2} u_{v}
$$

VI-7

The vapor mass flow rate per unit area is obtained from Equations VI-6 and VI -7, thus

$$
\dot{M}=e_{r} \frac{\pi}{16}\left[\frac{\sigma 4}{\rho_{r} 1_{0}}\right]^{1 / 2}\left[\frac{\rho_{L}}{\rho_{L}+\rho_{r}}\right]^{1 / 2}
$$

Expressing the wave length $\Lambda_{0}$ In Equation VI-8 in terms of the wave number $m_{0}$, It follows that

$$
\dot{M}=\rho_{v} \frac{\pi}{24} \frac{3}{\sqrt{2 \pi}}\left[\frac{\sigma m_{0}}{\rho_{v}}\right]^{1 / 2}\left[\frac{\rho_{L}}{\rho_{L}+\rho_{r}}\right]^{1 / 2} \quad \text { vI -9 }
$$

For liquids at saturation temperature the only energy requirement of the system is the energy needed to generate the vapor flux density given by Equation VI-9. Therefore the critical heat flux density in pool boiling from a horizontal surface is

$$
q=L \rho_{v} \frac{\pi}{24} \frac{3}{\sqrt{2 \pi}}\left[\frac{\sigma m_{0}}{\rho_{v}}\right]^{1 / 2}\left[\frac{\rho_{L}}{\rho_{L}+\rho_{v}}\right]^{1 / 2}
$$

VI-10 
155

It was seen in Section V-2 that experimental data supported the assumption concerned with the spectrum of unstable disturbances given by Equation V-2. Because of this spectrum the wave number $m_{0}$ can be determined within the range

$$
\left[\frac{g\left(p_{L}-p_{V}\right)}{\sigma}\right]^{1 / 2} \geqslant m_{0} \geqslant\left[\frac{g\left(p_{L}-p_{V}\right)}{3 \sigma}\right]^{1 / 2} \quad V I-11
$$

The critical heat flux density is determined therefore within the limits:

$$
\begin{aligned}
& \operatorname{Le} \frac{\pi}{24} \frac{3}{\sqrt{2 \pi}}\left[\frac{\sigma g\left(p_{L}-\rho_{v}\right)}{p_{v}^{2}}\right]^{1 / 4}\left[\frac{p_{L}}{p_{L}+l_{v}}\right]^{1 / 2} \geq q \geqslant \\
& q \geqslant \frac{\pi}{24} \frac{3}{\sqrt{2 \pi}} \frac{1}{(3)^{1 / 4}} L P_{v}\left[\frac{\sigma g\left(\rho_{L}-\rho_{v}\right)}{\rho_{V}^{2}}\right]^{1 / 4}\left[\frac{\rho_{L}}{\rho_{L}+e_{V}}\right]^{Y_{2}^{V I-12}}
\end{aligned}
$$

Equation VI-12 can be written as

$$
\begin{aligned}
& K_{1} L e_{v}\left[\frac{\sigma q\left(p_{L}-p_{L}\right)}{\rho_{v}^{2}}\right]^{1 / 4}\left[\frac{p_{L}}{p_{L}+p_{v}}\right]^{1 / 2} \geqslant q \geqslant \\
& q \geqslant K_{2}<p_{v}\left[\frac{\sigma g\left(p_{L}-p_{v}\right)}{\rho_{r}^{2}}\right]^{1 / 4}\left[\frac{p_{L}}{p_{L}+e_{r}}\right]^{1 / 2} \quad v I-13
\end{aligned}
$$

where

$$
K_{1}=\frac{\pi}{24} \frac{3}{\sqrt{2 \pi}} \Rightarrow 0.157
$$

$V I-14$ 
and

$$
K_{2}=\frac{\pi}{24} \frac{3}{\sqrt{2 \pi}} \frac{1}{(3)^{1 / 4}}=0.12
$$

The algebraic mean is, therefore

$$
K_{m}=0.138
$$

VI-16

A convenient average value for the critical heat flux can be obtained by replacing $m_{0}$ in Equation VI-10 by the upper limit obtained from Equation VI-II, and by approximating the numerical constant $3 / \sqrt{2 \pi}$ by unity. The resulting equation is given by

$$
q=\frac{\pi}{24} L e_{v}\left[\frac{\sigma g\left(\rho_{L}-\rho_{v}\right)}{\rho_{v}^{2}}\right]^{1 / 4}\left[\frac{\rho_{L}}{\rho_{L}+P_{v}}\right]_{V I-17}^{1 / 2}
$$

The value of the numerical constant in this case is:

$$
K=\frac{\pi}{24}=0.131
$$

In the section which follows, the theoretical results will be compared with experimental data. 
VI-2. The Critical Heat Flux - Comparison of Theoretical with Experimental Results

It is assumed that in transitional boiling changes In heat transfer rates are assoclated with changes of frequency only. We shall verify this assumption first. According to this postulate and to Equation V-7, the following relation should hold in transitional boiling:

$$
\frac{q_{1}}{q_{2}}=\frac{f_{1}}{f_{2}}
$$

where the subscripts 1 and 2 refer to two different operating conditions.

Westwater and Santangelo have reported that for an overall temperature difference $T_{w}-T_{0}=133^{\circ} \mathrm{F}$, and a heat transfer coefficient $h=164 \mathrm{Btu} / \mathrm{hr} \mathrm{ft}^{2} \mathrm{o}_{\mathrm{F}}$, the frequency was 84 bursts per second per inch length of tube. Whereas at a heat flux of $q=5470 \mathrm{Btu} / \mathrm{hr} \mathrm{ft}^{2}$, the frequency was 22 bursts per second per inch length. Substituting these values into Equation VI-19 it follows that

$$
\begin{aligned}
& \frac{q_{1}}{q_{2}}=\frac{21800}{5470}=3.98 \\
& \frac{f_{1}}{f_{2}}=\frac{84}{22}=3.82
\end{aligned}
$$


158

As a further check of the hypothesis the ratio of the frequencies computed from the analysis will be compared with the ratio of the maximum and minimum heat flux determined from experiments.

The frequency of the bursts of vapor at the critical heat flux is obtained from the equation of contenuity

$$
\frac{4 \pi}{3}\left(\frac{\Lambda_{0}}{4}\right)^{3} f_{c}=\pi\left(\frac{\Lambda_{0}}{4}\right)^{2} U_{v}
$$

$$
\text { VI-21 }
$$

hence

$$
f_{c}=\frac{3}{\Lambda_{0}} U_{v} \quad \text { VI -22 }
$$

Substituting Equation VI-6 into Equation VI-22 and neglecting the density ratio $e_{L}+e_{V} / e_{L}$ it follows that

$$
F_{c}=\frac{3}{\lambda_{0}}\left[\frac{\sigma}{\rho_{v}} \frac{4}{\lambda_{0}}\right]^{1 / 2}
$$$$
\text { VI-23 }
$$

The two frequencies which correspond to the disturbances $\Lambda_{01}$ and $\Lambda_{02}$ are obtained by substituting alternately Equation IV -4 and IV-5 into Equation VI-23, thus

$$
f_{\text {col }}=\frac{6}{\sqrt{2 \pi}} \frac{1}{\lambda_{01}}\left[\frac{\sigma g\left(\rho_{L}-\rho_{v}\right)}{\rho_{v}^{2}}\right]^{1 / 4} \quad \text { VI-24 }
$$


159

$$
F_{\mathrm{CO} 2}=\frac{6}{\sqrt{2 \pi}} \frac{1}{(3)^{1 / 4}} \frac{1}{\Lambda_{02}}\left[\frac{\sigma g\left(\rho_{L}-\rho_{v}\right)}{\rho_{v}^{2}}\right]^{1 / 4} \quad \text { vI -25 }
$$

The corresponding frequencies at the minimum heat flux are given by Equation $\mathrm{V}-16$; thus, after some rearrangement

$$
\begin{array}{ll}
f_{m 01}=\frac{2 \sqrt{2 \pi}}{3} \frac{1}{\lambda_{01}}\left[\frac{\sigma g\left(\rho_{L}-\rho_{v}\right)}{\rho_{L}^{2}}\right]^{1 / 4} & v I-20 \\
f_{m 02}=\frac{2 \sqrt{2 \pi}}{3}(3)^{1 / 4} \frac{1}{\Lambda_{02}}\left[\frac{\sigma g\left(\rho_{L}-\rho_{v}\right)}{\rho_{L}^{2}}\right]^{1 / 4} & \text { VI-27 }
\end{array}
$$

The ratio of the frequencies of vapor release at the critical and minimum heat flux densities are then given by

$$
\frac{f_{\text {col }}}{f_{\text {mol }}}=\frac{g}{2 \pi} \sqrt{\frac{\rho_{L}}{\rho_{V}}}
$$

$$
\text { VI -28 }
$$

and

$$
\frac{f_{C O 2}}{f_{\text {mat }}}=\frac{q}{2 \pi \sqrt{3}} \sqrt{\frac{\rho_{L}}{\rho_{r}}}
$$$$
\text { VI-29 }
$$ 
The critical and the minimum heat flus densitfes for boiling methanol were reported by Westwater and Santangelo (1). Substituting the theoretical and experimental values in Equation VI-19 the following result is obtained

Analysis

Equation VI-28 $\frac{E_{\mathrm{col}}}{E_{\mathrm{mol}}}=35.3$

Equation VI-29 $\frac{f_{\mathrm{co} 2}}{\mathrm{f}_{\mathrm{mo} 2}}=20.6$
Experiment

$$
\frac{q_{\text {crit }}}{q_{\text {min }}}=\frac{172000}{5470}=31.4
$$

It appears from this comparison and from Equation VI-20 that experimental results tend to support the hypothesis that in transitional bolling changes of heat transfer are assoclated with changes of frequency only. We shall compare now the critical heat flux densities predicted by the theory with experimental data. Inssmuch as the square root term in Equations VI-12 and VI-17 is close to unity except in the nelghborhood of the thermodynamic critical state, it is seen that both Equations III-9 and III-12 are of the same form as Equation VI-12. The agreement of the heat transfer rates predicted by Equation III-9 with experimental data was discussed in Section III-3; this agreement is shown also on Figure III-3. Therefore, for a comparison of the present analysis with experiments it suffices to compare the value of the 
coefficients $K$ determined analytically with the values determined by Kutateladze and Borishanskil from experiments.

Analysis

Equation VI-13 $0.12 \leqslant K \leqslant 0.157$

Equation VI-17 $\mathrm{K}=\frac{\pi}{24}=0.131$
Experiment

Kutateladze

$$
K=0.16
$$

Borishanski1

$$
\mathrm{K}=0.13
$$

The values of the constant $K$ given by the theoretical limits

$$
\frac{\pi}{24} \frac{3}{\sqrt{2 \pi}}=0.157 \geqslant K \pi 0.12=\frac{\pi}{24} \frac{3}{\sqrt{2 \pi}} \frac{1}{(3)^{1 / 4}}
$$

are indicated on Figure III-3.

In Figure VI-1 the heat transfer rates predicted by Equation VI-17 are compared with experimental data for water by Kazalkova (108). Another comparison with experimental data for ethanol reported by Cichelli and Bonilla (82) is shown on Figure VI-2.

It appears from the above comparisons that this simple idealized system exhibits the detailed and general features of the phenomenon. It is interesting to note that the analysis predicts an inherent uncertainty in determining the exact heat flux. The width of this uncertainty band is approximately $\pm 14 \%$. It follows from the theory that a certain irreproducibility of the experimental results can be expected. The scatter of experi- 


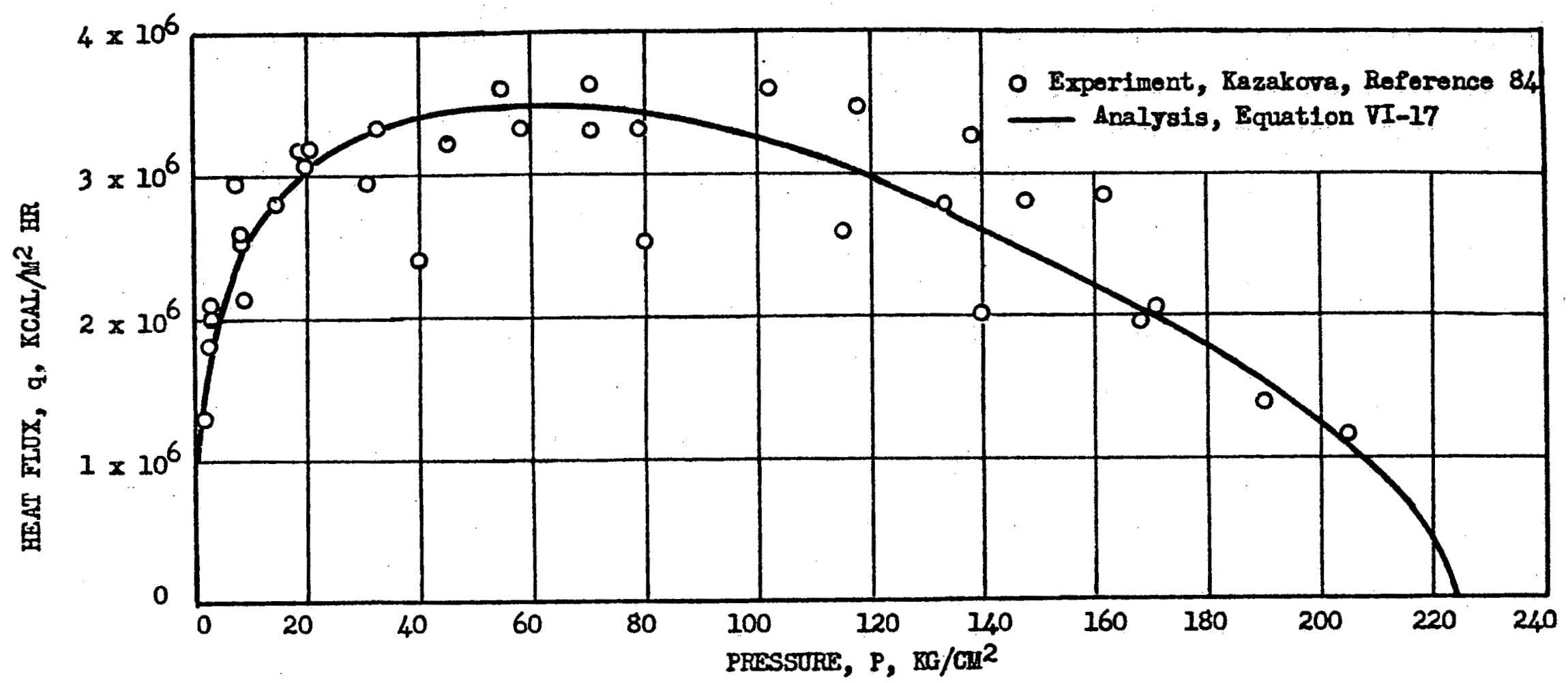

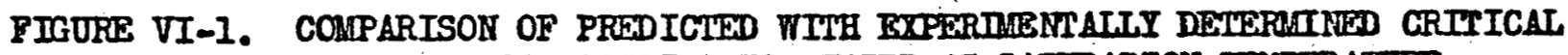
HEAT FLUX DENSITIES IN POOL BOILING FOR TATER AT SATURATION TMMPERATUFE 


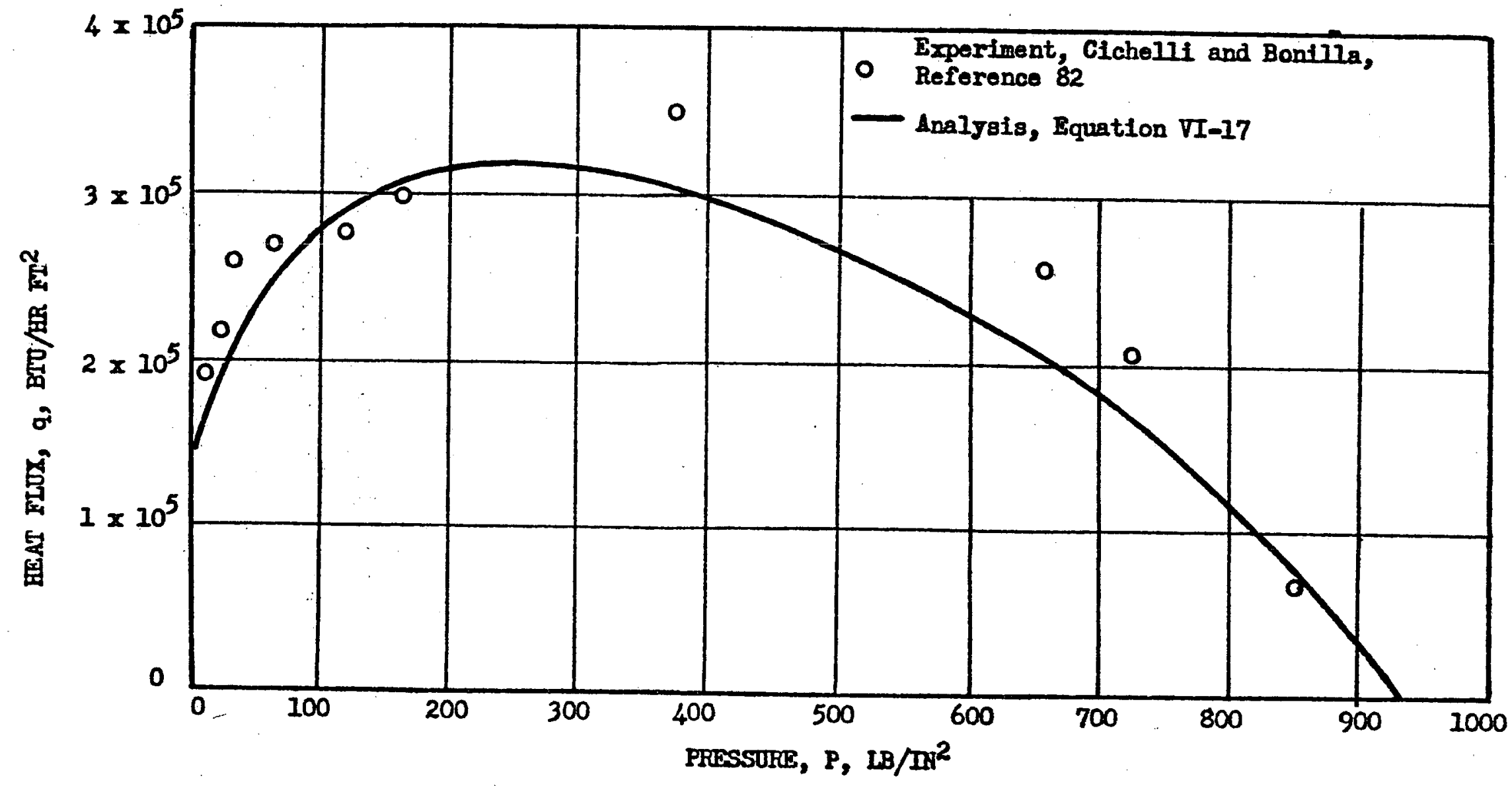

FIGURE VI-2, COMPARISON OF PREDICTED WITH EXPERTARMINLY DETERAINED CRTTICAL BEAT FLUX DEISITIES IN POOL BOIIING FOR ETHYL ALCOBOL AT SATURATION TEMPERATURE 
mental data is often reported in the literature. In the following section the analysis will be extended to transition boiling of subcooled liquids.

VI-3. The Critical Heat Flux in Pool Boiling of a Subcooled Liquid

Equations VI-12 and VI-17 which predict the critical heat flux were determined from stability considerations and an energy balance for liquids at saturation temperature. To extend the analysis to subcooled liquids a basic assumption will be made: The change from nucleate to transitional boiling is determined only by the hydrodynamic stability; in pool boiling, i.e., in the absence of a forced flow, the change occurs when the vapor flux attains a given value. Therefore, if the liquid is at saturation and the viscosity is neglected, the heat transferred across the solid surface is equal to the energy required for the generation of that particular vapor mass flow. Since the stability is the mechanical aspect of the problem it will be unaffected by whether the Iiquid is subcooled or not, but will depend only on the mass transport. However, as the heat flux across the solid surface is determined by an energy balance it will depend on the subcooling. Therefore all energy requirements will appear as additive terms to the energy needed for the generation of the critical vapor flow. The problem 
is reduced now to the determination of energy requirements associated with a bolling, subcooled liquid.

In transitional boiling the vapor-1lquid interface Is at saturation temperature; when the bulk liquid is subcooled, the problem is to determine the energy transferred from the interface to the bulk liquid. It was discussed in the preceding sections that in transitional bolilng vapor is periodically released from the Interface. Because of this release both the interface and the temperature distribution are periodically renewed. The process can be described therefore by the "discontinuous film model" which was discussed in section III-8. If the assumption is de that the "contact time" Is short compared to the ratio of the scale of turbulence to the intensity of turbulence, the energy will be transferred mainis by conduction. This is equivalent, therefore, to the assumption that the "depth of penetration" is small compared to the "scale of turbulence." Assuming a plane interface the energy problem is described by Equation II-2. In transitional boiling to a given heat flux there corresponds a given frequency. Consequently, the average heat flus from the interface to the bulk liquid is given by Equation III-I7 (instead of Equation III-18), thus

$$
q=2 \kappa \frac{\left(T_{S}-T_{L}\right)}{\sqrt{\pi a \tau}}
$$


166

where $\tau$ is the period. In References 87 and 88 the equations which correspond to Equations II-4 and VI-30 of this dissertation were multiplied by the factor $b=\pi / 2$ (see section II-1) in order to account for the distortion of the interface. Experimental data of Kutateladze and Schneiderman (109) indicate that this correction is not needed. The factor $b$ is omitted therefore from Equation VI-30. Expressing the period in terms of the frequency given by Equation VI-24, Equation VI-30 becomes

$$
q_{2}=2 \kappa \frac{\left(T_{S}-T_{L}\right)}{\sqrt{\pi a}} \sqrt{f_{c o l}} \quad \text { VI -SI }
$$

The heat transferred across the solid surface is obtained again from an energy balance, $i_{\text {.e., by adding Equations }}$ VI-17 and VI-3I. Consequently the critical heat flux in subcooled, pool boiling from a horizontal surface is given by

$$
q_{\text {CSS. }}=L_{0} \rho_{r} \frac{\pi}{24}\left[\frac{\sigma g\left(\rho_{L}-\rho_{V}\right.}{\rho_{r}^{2}}\right]^{1 / 4}+\frac{2 K\left(T_{s}-T_{L}\right)}{\sqrt{\pi Q}} \sqrt{f_{c 01}} \quad \text { VI -32 }
$$


267

where

$$
\begin{array}{ll}
f_{(0)}=\frac{6}{\sqrt{2 \pi}} \frac{1}{\Lambda_{01}}\left[\frac{\sigma g\left(\rho_{L}-e_{v}\right)}{\rho_{v}^{2}}\right]^{1 / 4} & \text { vI-33 } \\
\Lambda_{01}=2 \pi\left[\frac{\sigma}{g\left(\rho_{L}-\rho_{V}\right)}\right]^{1 / 2} & \text { VI-34 }
\end{array}
$$

In Figure VI-3 the heat transfer rates predicted by Equation VI-31 are compared to the experimental data reported by Gunther and Kreith (7) for water boiling at atmospheric pressure from a horizontal surface. A comparison is shown also with experimental results for ammonia and carbon tetrachloride reported by Bartz (110) and by Ellion (15). The experimental data of Kutateladze and Schneiderman are reported as the ratio $q_{c . s u b} / q_{c . s a t} \cdot$ In order to predict this ratio we divide Equation VI-32 by Equation VI-17, thus

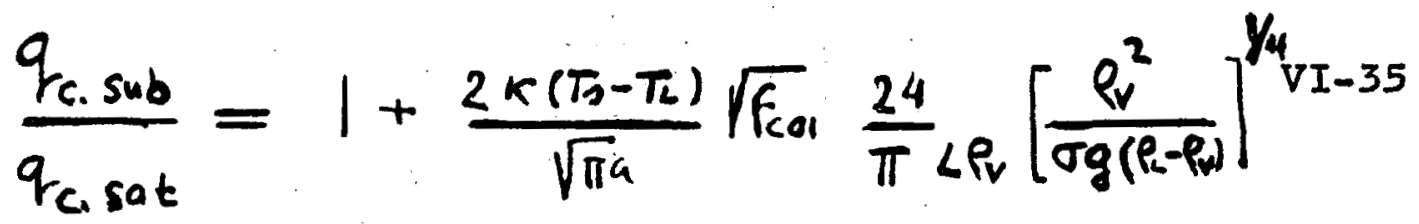




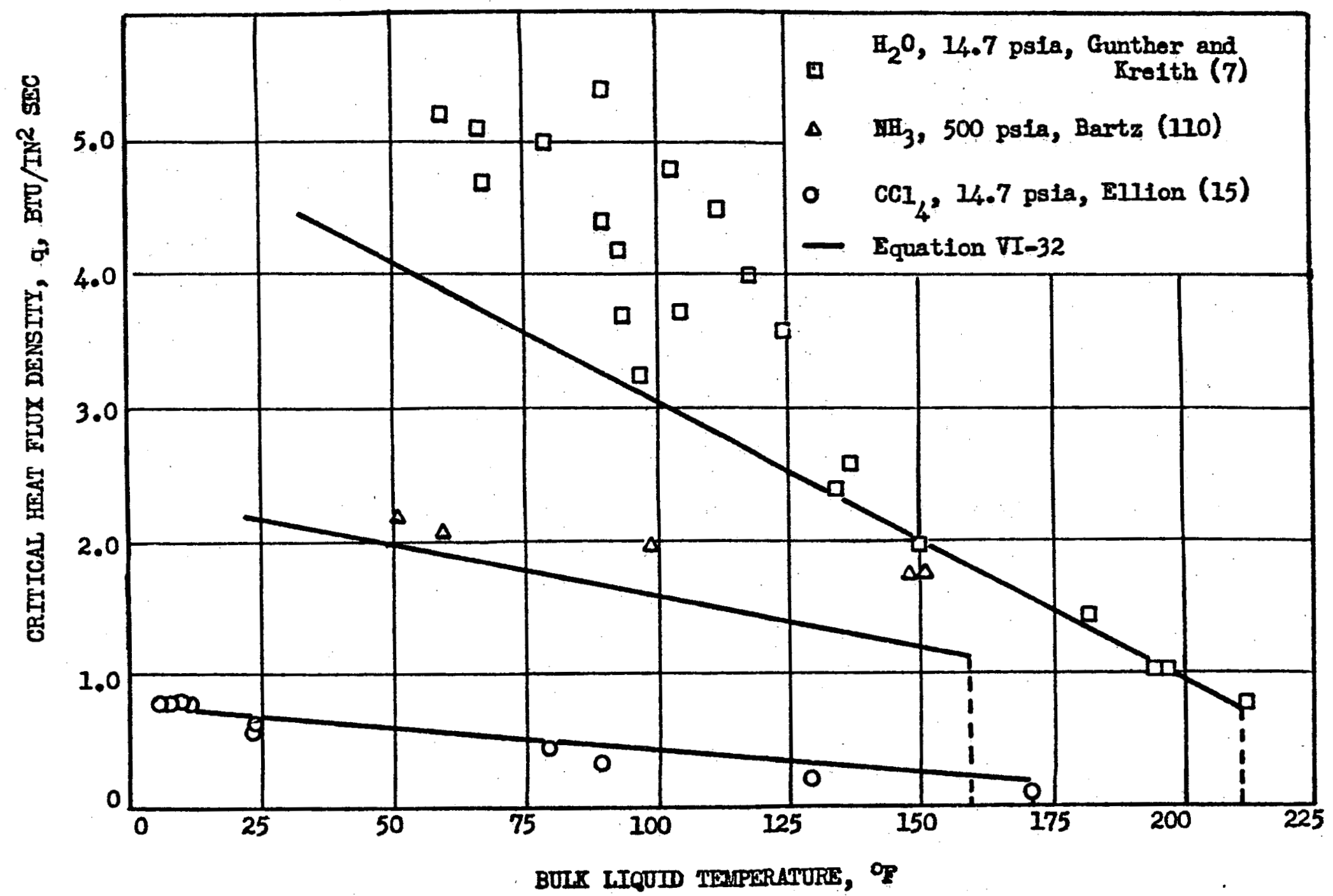

FIGURE VI-3. COMPARISON OR PREDICTED MITH EXPERTIMENTALLY DETERMINED CRITICAL HBAT TRANSTER RATES IN POOL BOIIITG OF SUBCOOLED LIQUIDS 
On Figures VI-4 and VI-5, Equation VI-34 is plotted together with the experimental data of Kutateladze and Schneiderman. The agreenent appears to be satisfactory.

It should be noted that the interface in subcooled boiling is not plane, and the treatment of the interface as though it were a plane is introduced as the simplest idealization. However, it will follow that no matter what geometry is postulated, the form of the resulting solution is unchanged and only the numerical constant will be affected in Equation VI-33. This change will alter each of the slopes of Figures VI-3, 4 and 5 in the same way. The group $k \sqrt{f / a}$ will still determine the relative slopes for different liquids and the retios of the slopes will be unaffected by the numerical constant. The agreement shorn by Figures VI-3, 4 and 5 concerming absolute as well as relative slopes serves to indicate that the conception of the process is proper. Similar remarics apply to the use of a "contact time."

As a closing remark we note that in some experiments, in order to maintain the subcooling, the liquid is circulating slowly. The velocity of circulation is low, so that the process can be considered pool boiling. When the subcooling is low, bubbles will depart from the interface and will be removed by the circulating liquid. The mass of vapor is replaced by an equivalent 


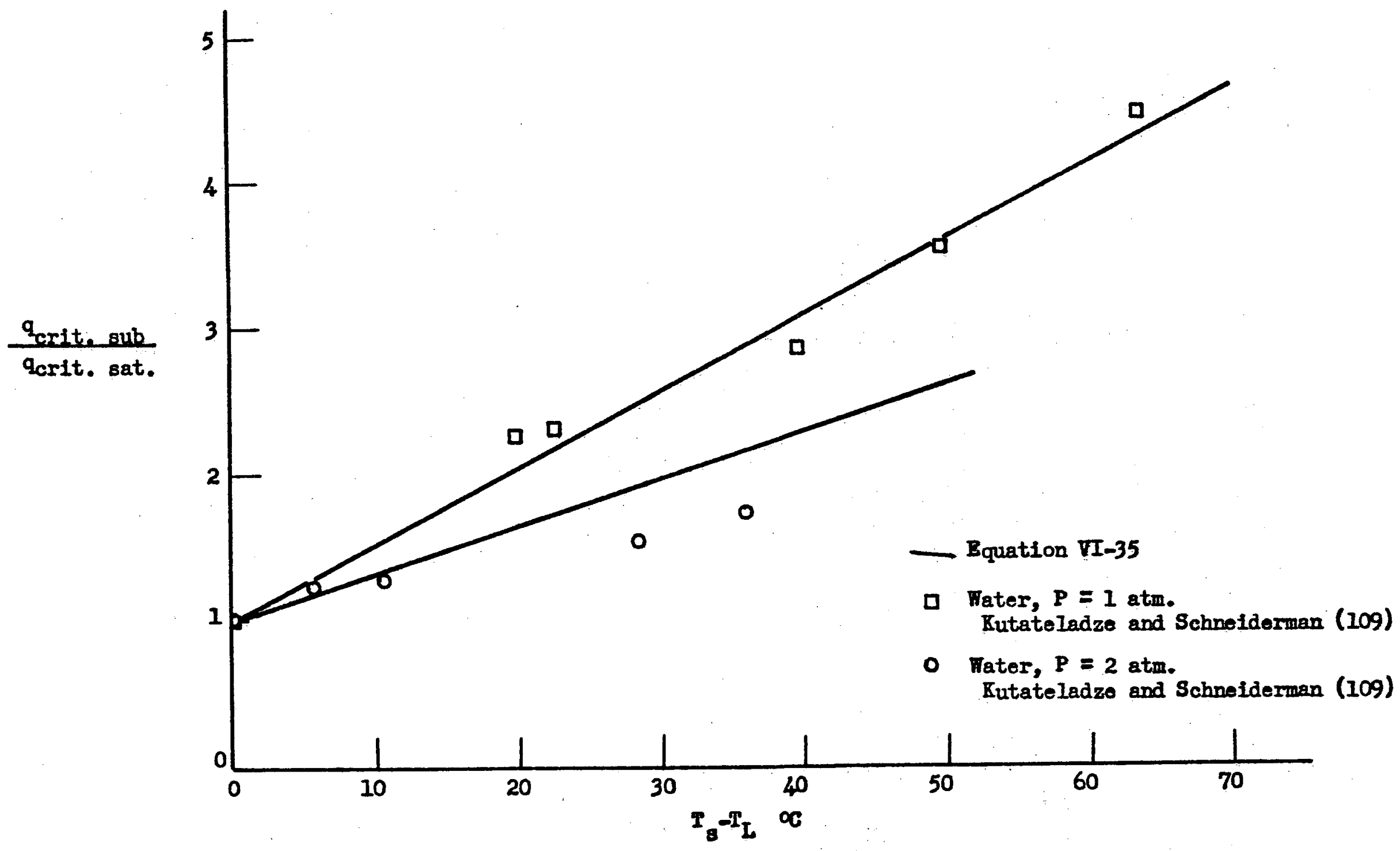

FIGURR VI-4. COMPARISON OP PREDICTED FITH EXPERDMENTALLY DETERMINED CRITICAL HEAT TRANSEER RATES IN POOL BOILING OF SUBCOOLED TIATER 


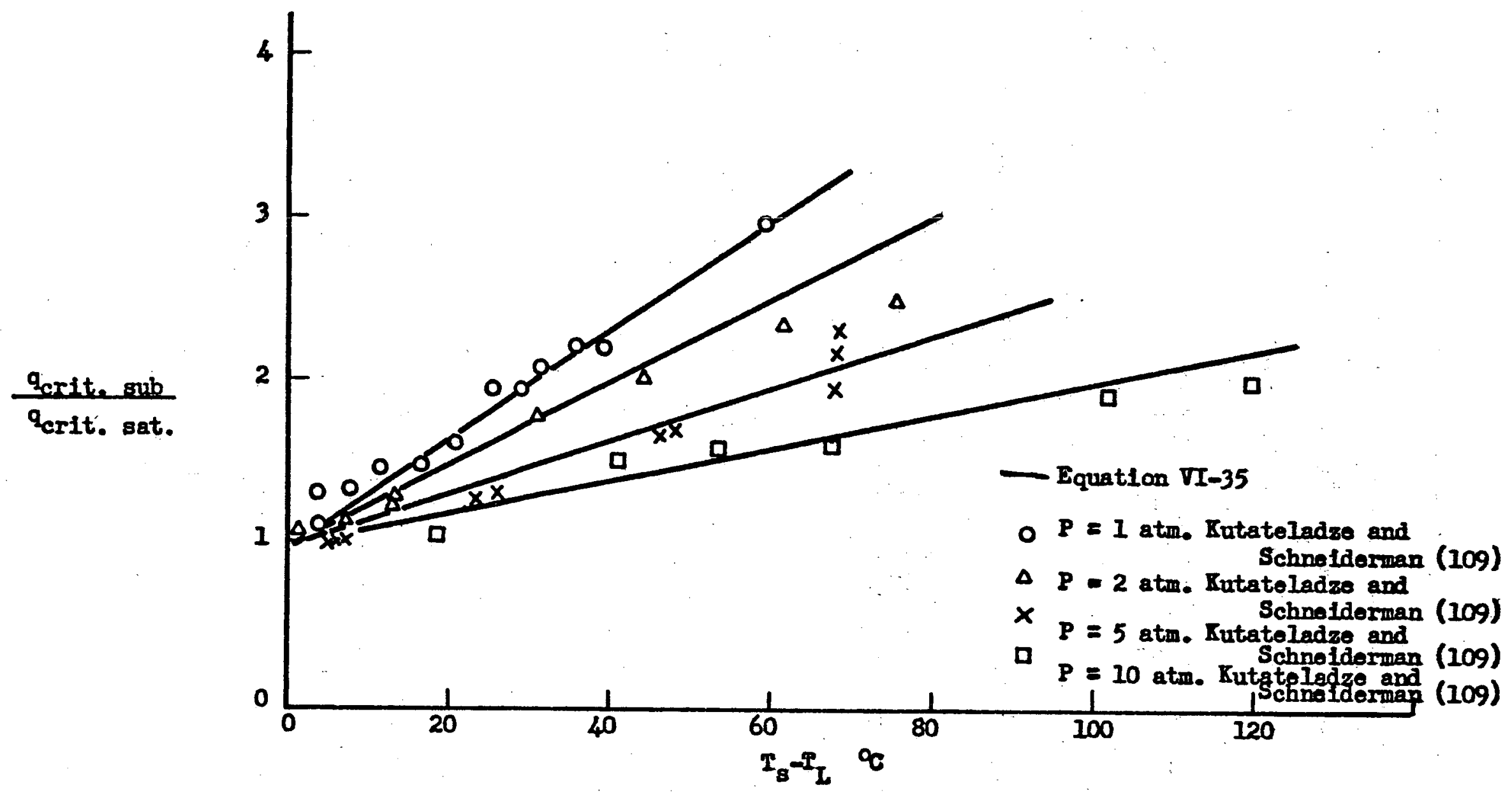

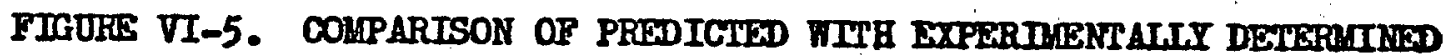
CRTIICAL HEAT TRANSFER RATES IN POOL BOTIING OF SUBCOOLED ETHYL ALCOHOL AT VARIOUS PRESSUTHES 
mass of liquid which, as the bulk temperature is aubcooled, has to be heated up to saturation temperature first before the evaporation can occur. From Equation VI-17, the critical vapor mass flow rate $1_{s}$ given by

$$
\dot{M}=e_{v} \frac{\pi}{24}\left[\frac{\sigma g\left(e_{L}-e_{v}\right)}{e_{v}^{2}}\right]^{1 / 4}
$$

The enthalpy change $\Delta H$, of the liquid thus becomes

$$
\Delta H=\left[\frac{\pi}{24} e_{v}\left[\frac{\sigma g\left(e_{L}-e_{v}\right)}{e_{r}^{2}}\right]^{1 / 4}\right] c\left(T_{S}-T_{L}\right)
$$

Because in the experiments of Gunther and Krelth and of Kutateladze and Schnelderman the Ilquid did not circulate, Equation VI-37 was not Included in the energy balance. 
SUMMARY AND CONCLUSIONS

Chapter I. - A Review of Nucleate Bolling

The problems of nucleation from a solid surface and the dymamics of a bubble departing from the heating surface were considered. The characteristics of nucleate bolling and the relation between the heat flux density, the aurface roughness and the liquld superheat temperature difference were discussed. It was seen that, in nucleate boiling, the heat flux density is not a single valued function of the temperature; in order to determine the $q$ - T relation it is necessary to specify the characteristics of the surface.

Chapter II. - The Problem of Bubble Growth

The problem of bubble growth in a superheated liquid was discussed. It was pointed out that the original conceptual and mathematical formulation of the problem as given by Bosnjakovic and Jakob contain the essential features of the phenomenon.

The theory of Bosnjakovic and Jakob was extended to include the effects of a non-unfform temperature field; the growth rates predicted by Equations II-I7 and II-30 are in satisfactory agreement with experimental data. 
These equations are not adequate for predicting the collapse of a bubble in subcooled boiling. Experimental data Indicate that the collapse can be approximated by an 1sothermal process; it can be predicted by the solution of Rayleigh's equation, 1.e., by Equation II-33. Experimental data for bubbles growing and collapsing in subcooled boiling can be approximated therefore by considering the growth and collapse process separately. The growth and collapse equations are matched at the maximum bubble radius.

If a bubble is suddenly introduced into a subcooled liquid, or if the saturation temperature is suddenly increased (by increasing the pressure), then, initially, the thickness of the thermal boundary layer is much smaller than the bubble radius. Under these conditions the bubble collapse rate is given by Equation II-35.

Experimental data tend to indicate that the diameter of an active nucleating cavity can be related to the heat flux and superheat temperature by the simple relation given by Equation II-26. Further experimental investigations are needed to verify this relation.

Chapter III. - Hydrodynamic Aspects of Nucleate Boiling

The similarity between bubble formation at an orifice and bubble formation in nucleate boiling was pointed out and discussed. Using this similarity an 
equation (Equation III-4 or III-5) was derived for the product bubble diameter times frequency of bubble emission in nucleate bolling. Equation III-5 was shown to be in good agreement with experimental data. From Equation III-4 it is possible to estimate the meximum frequency of bubble release in nucleate boiling. The simflarity also indicates the possibility of relating (by Equation III-1 or III-6) the diameter, $D_{\mathrm{d}}$, of a bubble departing from a heated horizontal surface to the radius, $I_{0}$, of the nucleating cavity. From auch a relation it would be posstble to estimate the spectrun of departing bubbles in terms of a distribution of nucleating cavities. Further experiments are needed to verify this relation. The hydrodynamic aspects of nucleate boiling and the region of the critical heat flux were analyzed. It was seen that nucleate boiling at low heat flux densities Is characterized by the presence of single bubbles; whereas the region of the critical heat flux is characterlzed by the presence of vapor columns and large patches of vapor.

The simllarity between nucleate boiling and the bubbling of a gas from a porous plate was discussed. This similarity indicated that the change from nucleate to transitional boiling is a hydrodynamic phenomenon known as "flooding". It occurs when the stability of 
two-phase flow is disrupted. It was concluded that extating correlations of experimental data can be understood only if the critical heat flux is interpreted as a "flooding" phenomenon.

Chapter IV. Hydrodynamic Aspects of Transitional Boiling

The hydrodymamic stability of a plane interface was analyzed and experimental results pertaining to Taylor Instability were discussed. The hydrodynamic aspects of transitional boiling were qualitatively analyzed and the phenomenon was interpreted in terms of Taylor instability. It was concluded that, as a consequence of Taylor instability, a definite geometrical configuration can be expected in transitional bolling. As a consequence of this definite geometrical configuration, a release of bubbles occurs at regular intervals. At a constant pressure the factors which influence the geometry remain invarlant. Consequently, in transitional boiling, changes In heat transfer rates are assoclated only with changes In the frequency of bubble release. The critical heat flux and the minimum heat flux in transitional bolling correspond, therefore, to the maximum and minimum allowable frequencies of the system. 
Ghapter V. - The Minimum Heat Flux in Transitional

\section{Boiling from a Horizontal Surface}

The diameters of the vapor slugs in transitional boiling were estimated by considering the critical and the "most dangerous" wave length (Equation $\mathrm{V}-2$ ) given by Taylor instability. It was noted that, at the minimum heat flux density, the dynamic effect of the vapor phase upon the motion of the interface is small. It was concluded therefore that the rate of penetration of the vapor into the Iiquid cannot be faster than the rate which would be predicted from Taylor instability. Because this rate of penetration is initially exponential and later reaches a constant value, two equations (Equation $V-11$ and $V-16$ ) were derived for the frequency of bubble release. Using the two frequencies and malking an energy balance, two equations (Equation $V-13$ and $V-19$ ) were derived for the minimum heat flux density in transitional boiling. The theoretical results were found to be in good agreement with experimental data.

Chapter VI. - The Critical Heat Flux Density from a Horizontal Surface

The critical heat flux density was analyzed by considering transitional boiling. Because of large evaporation rates, at the critical heat flux, the dynamic effect of the vapor upon the motion of the 
11quid-vapor interface 18 important. The problem was formulated by considering the combined effect of Taylor and Helmholtz Instabilities. Analytical expressions were derived which perut the prediction of the critical heat flux density in pool bolling of saturated or subcooled 1iquids. The numerical values of the empirical constants whfch appear In the Kutateladze and Borishanslefl criteria for the critical heat flux are derived from the theory. The theoretical reaults are in good agreement with experimental data. 


\section{BIBLIOGRAPHY}

1. Westwater, J. W. and J. G. Santangelo, "Photographlo Study of Boiling," Ind. Engr. Chem. 47, 1955, 1605.

2. Jakob, M. and W. Fritz, "Verguche uber den Verdampfungsvorgang," For schung, 2 Bd. Heft 12, 1931, 435.

3. Jalkob, M., "Kondensation and Verdampfung," Zetschr. d. Ver. Deutsch. Ingr., Bd. 76, 1932, 1161.

4. Jakob, M. and W. Iinke, "Der Wärmellbergang von elner Waagerechten Platte an Siedenes Wasser," Forschung, 4 Bd. , Heft 2, 1933, 75.

5. Jakob, M., and W. Linke, "Der Wärmellbergang beim Vergampfen von Flussigkelten an Senkrechten und Waagerechten Flächen," Phys. Zeltsch., 36, 1935, 267.

6. Jakob, M., "Heat Transfer in Evaporation and Condensation," Mech. Engr., 58, 1936, 643 and 729.

7. Gunther, F. C. and F. Kreith, "Photographle Study of Bubble Formation in Heat Transfer to Subcooled Ilquids," Heat Transfer and Fluid Mechanics Inst., Berkeley, 1949, 113.

B. Rohsenow, W. and J. Clark, "A Study of the Mechenlsw of Boiling Heat Transfer," Trans, A.S.M.E., 73, $1951,609$.

9. Banleoff, S. G., "Ebullition from Solid Surfaces in the Absence of Pre-existing Gaseous Phases," Trans. A.S.M.E., 79, 1957, 735 .

10. Clark, H. B., P. H. Strenge and J. W. Westwater, "Active Sitea for Nucleate Boiling," Preprint No. 13, A.I.Ch.E.-A.S.M.E. Heat Transfer Conference, Chicago, 1958 .

11. Dzhandzhgava, Sh. G., "Investigation of the Formation of Bubbles and of the Superheat," Dok. Ak. Nauk S.S.S.R., 70, No. 3, 1950,417 . 
12. Nesis, E. I., "Boiling under Real Conditions," Zh. Tekh. F1z., 22, 1952, 1506.

13. Courty, 0., "Surface Variables in Nucleate Boiling," Ph. D. The sis, Purdue Univ., Lafayette, Ind., 1952.

14. Courty, C. atid A. Foust, "Surface Varlables in Nucleate Boling," Chem. Engr. Progr. Symposium, Serles $51,1955,1$.

15. Ellion, M. E., "A Study of the Mechanism of Bolling Heat Tranafer," Ph. D. Thesis, Calif. Inst. Tech., 1954, Jet Prop. Lab Rept. Memo. 20-88, Celif. Inst. Tech., 1954.

16. Griffith, P. and J. D. Wallis, "The Role of Surface Conditions in Nucleate Boiling," Tech. Rept. No. 14, Mass. Inst. Tech., Dec., 1958.

17. Bankoff, S. G., "The Entrapment of Gas in the Spreading of a Liquid Over a Rough Surface," A.I.Ch.E. Journal, $4,1958$.

18. Bankoff, S. G., "The Prediction of Surface Temperatures at Inciplent Boiling," Preprint 4, A.I.Ch.E.-A.S.M.E. Heat Transfer Conference, Chicago, 1958.

19. Sabersley, R. H. and C. W. Gates, "On the Start of Nucleation In Boiling Heat Transfer," Jet. Propulsion, $1955,67$.

20. Treschov, G. G., "Experimental Investigation of the Mechanism of Heat Transfer in Surface Boiling," Teploenergetika, 3, No. 5, 1957, 44.

21. Wark, J. W., "The Physical Chemistry of Flotation," J. Phys. Chem., 37, 1933, 623.

22. Fritz, W., "Berechnung des Maximalvolumens von Dempfblasen," Phys. Zeitsch., 36, 1935, 379.

23. Bashforth, F. and C. Adams, An Attempt to Test the Theories of Capillary Action, Cambridge Univ. Press, 1883.

24. Fritz, W. and W. Ende, "Verdampfungsvorgang nach Kinematographischen Aufnahmen an Dampfblasen," Phys. Zeitsh., 37, 1936, 401. 
25. Westwater, J. W. and A. S. PerleIns, Measurements of Bubbles Formed In Bolling Methanol," A.E.Ch.E. Jour., 2, 1956, 471 .

26. Donald, M. B. and F. Haslam, "The Mechanism of the Transition from Nucleate to F1lm Boiling," Chem. Engr. Sc., 8, 1958, 287.

27. Yamagata, K. and $K$. Nishikawa, "Nucleate Bolling of Water on the Horlzontal Heating Surface," Memoirs of the Faculty of Engr., Kyushu Univ., 15, No. 1, 1955.

28. Aver1n, E. K., "Effect of Materfal and Machining of Surface on Heat Transfer to Bolling Water," Izv. Akad. Naulk S.S.S.R., Otd. Teleh. Naule No. 3, A.E.R.E. Tr. 562, 1954, 116 .

29. Zysina-Molozhen, I. M., "Some Data on the Number of Centers of Vaporization in Bolling on Industrial Heating Surfaces," published in Problems of Heat Transfer During a Change of State, G. E. I., Moscow, 1953.

30. Bosnjalcovic, F., "Verdampfung und FI ilssigheitsliberhitzung," Tech. Mech. und Therm., Bd. 1, 1930, 358.

31. Prüger, W., "Dle Verdampfungsgeschwindigkeit der Fllssiglkeiten," Zeitsch. fur Physik, B-115, 1940, 202.

32. Plesset, M. and S. A. Zwicle, "A Non-Steady Diffuaion Problem with Spherical Symetry," J. Appl. Phys,, 23, 1952, 95.

33. Plesset, M. and J. A. Swicle, "The Growth of Vapor Bubbles in Superheated Liquids," J. Appl. Phys., 25, 1954, 495.

34. Romie, F., "The Growth of Bubbles in Superheated Liquids," Memorandum, Dept. of Engr., Univ.. of Calif, Ios Angele s, 1952; see also M. I. Greenfield, et al., "Density Transients in Volume Heated Bolling Systems," Rept. 53-17, Dept. of Engr., Univ. of Calif., Ios Angeles, 1953.

35. Forster, H. K. and N. Zuber, "Growth of a Vapor Bubble in a Superheated Liquid," J. Appl. Phys., 25, 1954, 474.

36. Dergarabedian, P., "The Rate of Growth of Vapor Bubblea in Superhe ated Water," J. Appl. Mech., 20, 1953, 537. 
37. Birkhoff, G., R. S. Margulies, and W. A. Horning, "Spherical Bubble Growth," Phys. of Fluids, I, 1958, 201.

38. Scriven, L. E., "On the Dynamics of Phase Growth," Report $P$ 659, Shell Development Co., Emeryville, California, 1958.

39. Savic, P., "The Cooling of a Hot Surface by Drops Boiling in Contact with It," Nat. Res. Council of Canada, Div. of Mech. Engr., Rept. No. MT-37, April, 1958.

40. Carslaw, H. S. and J. C. Jaeger, Heat Conduction in Solids, Oxford Univ. Press

41. Treschow, G. G., "Experinental Investigation of the Mechanism of Heat Transfer in Surface Bolling," Teploenergetika, 3, 1957, 44 .

42. Zmola, P., "Investigation of the Mechanism of Boiling In Liquids," Ph.D. Thesis, Purdue Univ., Lafayette, Ind., 1950.

43. Gunther, F., "Photographic Study of Surface Boillng Heat Transfer to Water with Forced Convection," Trans. A.S.M.E., 73, 1951, 115.

44. Griffith, P., "Bubble Growth Rates in Boiling," Ph.D. The sis, Mass. Inst. Tech., 1956, Tech. Rept. No. 8, Mass. Inst. Tech., 1956, Trans. A.S.M.E., 80, 1958 .

45. Bankoff, S. G. and R. D. Mikesell, "Bubble Growth Rates in Highly Subcooled Nucleate Boiling," Preprint 2, A.S.M.E-A.I.Ch.E. Heat Transfer Conference, Chicago, 1958.

46. Banleoff, S. G. and R. D. Mikesell, "Growth of Bubbles in a Liquid of Initially Nonuniform Temperature," Paper 58-A-105, A.S.M.E. Annual Meeting, New York, 1958.

47. Bankoff, S. G., Colahan, W. J., and D. R. Bartz, "Summary of Conference on Bubble Dynamics and Boiling Heat Transfer Held at the Jet Propulsion Laboratory, June 14 and 15, 1956," Jet Propulsion Lab Memo. No. 20-137, Calif. Inst. Tech., 1956. 
48. Ple sset M. and S. A. ZwLlel, "On the Dynamics of Vapor Bubbles in Ifquida," J. Math. and Phys., 33 , $1955,308$.

49. Zuber, N., "Bolling Heat rransfer to Subcooled Ilquids " Memor andum, Dept. of Engr., Univ. of Calif. at Ios Angeles, 1956.

50. Lamb, H., Hydrodynamtes, Dover Publ., New Yorle, 1957.

51. Rohsenow, W., "A Method of Correlating Heat Transfer Date for Surface Boiling of Liquids," A.S.M.E. Trans, , 74, 1952, 969.

52. Rohsenow, $W$. "Heat Transfer with Evaporation," Heat Transfer Symposium, University of MichIgan, 1952.

53. Kutateladze, S. S., Heat Transfer in Bolling and Condensation, Mashgiz, Moscow, IY52.

54. Kruzhflin, G. N., "Generallzation of Experimental Data of Heat Transfer to Bolling Liquids in Free ConvectIon," Izv. Akad. Nauk S.S.S.R. No. 3, 1949, 701, A.E.G. Translation RT 2542.

55. Kruzhilin, G. N. , "Heat Transmission from a Heating Surface to a Boiling One-component Liquid In Free Convection," Izv. Alkad. Naule, Otd. Telkh. Nauk., 1948, 967, A.E.C. Translation RT 2792.

56. Sterman, I. S., "Theory of Heat Transfer in Bolling Llquids," Zh. Tekh. F1z., 23, 1953, 342.

57. Sternan, L. S., "Investigation of Heat Exchange by Boiling Liquids in Tubes," Zh. Tekh. Fiz., 24, 1954, 2046, A.E.R.E. Translation 565.

58. Slegel, R. and G. Usiskin, "A Photographic Study of Boiling in the Absence of Gravity," A.S.M.E. Paper 59-AV-37, 1959 Aviation Conference, Los Angeles.

59. Van Krevelen, D. W. and P. J. Hoftijzer, "Studfes of Gas Bubble Formation, Calculation of Interfacial Area In Bubble Cont actors," Chem. Engr. Progress, $46,1950,29$.

60. Siemes, W. "Über die Erzengung und den Aufstieg von Gasblasen in Fillsaiglesten," Chem. Ingr. Technile, 26, 1954,479 . 
61. Siemes, W., "Gasblasen in Flüssigleiten", Chem. Ingx, Techn., 26, 1954, 614.

62. Siemes, W. and J. F. Kaufman, "Dle Periodische Enstehung von Gasblasen an Dusen," Chem. Engr. Sc., $5,1956,127$.

63. Hughes, R. R., Handlos, A. E., Evans, H. D., and R. I. Maycocle, "The Formation of Bubbles at simple Orifices," Chem. Engr. Progress, 57, 1955, 557.

64. Davidson, L. and E. Amicl, "Formation of Gas Bubbles at Horizontal Orifices," A.I.Ch.E. Journal, 2, 1956, 336.

65. Peebles, F. N. and H. J. Garber, "Studies on the Motion of Gas Bubbles in Liquids," Chem. Engr. Progress, 49, 1953, 88.

66. Kutateladze, S. S. and M. A. Styrikovich, Hydraulics of Air-Liquid Systems, "Gosenergoizdat, Moscow, 1958.

67. Jakob, M., Heat Transfer, Vol. 1, Wiley, New York, 1949.

68. Verschor, $H_{6}$, "Some Aspe cts of the Motion of a Swarm of Gas Bubbles Rising Through a Vertical Liquid Volumn," Trans. Inst. Chem. Engr., 28, 1950, 52.

69. Kölbel, H. and W. Siemes, "Eigenschaften von Blasensäulen," Dechema-Monographien, Bd. 29, 1957, 334.

70. Siemes, W. and M. Borchers, "Begassung von Flüssigdurch Porose Sintermetalle," Chem. Ingr. Tech., 28, 1956, 787 .

71. Houghton, G., McLean, A. M., and P. D. Ritchie, "Mechanism of Formation of Gas Bubble-Beds," Chem. Engr. Sci., 7, 1957, 40 .

72. Petricle, M., "Two Phase Air Water Flow Phenomena," Argonne Nat. Lab. Rept. 5787, 1958.

73. Wallis, C. and P. Griffith, "Liquid and Gas Distribution in a Two-Phase Boiling Analogy," Tech. Rept. No. 13, Mass. Inst. Tech.,. December 1958. 
74. McAllister, R. A., McGinnis, P. H. and C. A. Plank, "Perforated Plate Performance," Chem. Engr. Sc., 9, $1958,25$.

75. Elgin, J. G. and F. B. Welss, "Lfquid Holdup and Flooding fin Packed Towers," Ind. Eng. Chem., 31, 1939, 435 .

76. Bonilla, C. and C. W. Perry, "Heat Transmission to Boiling Binary Liquid Mixtures," Tran. Am. Inst. Chem. Engr., 37, 1941, 685.

77. Kutateladze, S. S., "On the Transition to F1lm Boiling under Natural Convection," Kotloturbostroenie, No. $3,1948,10$.

78. Kutateladze, S. S., "A Hydrodymamic Theory of Changes in the Bolling Process under Free Conduction," Izv. Akad. Nauk, S.S.S.R., Otd. Tekh. Naule, No. 4, 1951, 529 .

79. Borishanskli, V. M., "An Equation Generalizing Experimental Data on the Cessation of Bubble Boiling in a Large Volume of Liquid," Zhur. Tekh. Fiz., 25, 1956, 252. (See Soviet Physics - Technical Phys., I, No. 2, 438.)

80. Borishanskil, V. M., "On the Problem of Generalizing Experimental Data on the Cessation of Bubble Boiling in a Large Volume of Liquids," Ts. K.T.I., 28, Moscow, 1955.

81. Rohsenow, W. and P. Griffith, "Correlation of Maximum Heat Flux Data for Bolling of Saturated Iiquids," Preprint No. 9, A.S.M.E.-A.I.Ch.E. Heat Transfer Symposium, Louisvilie, Ky., 1955.

82. Cichelli, M. T. and C. F. Bonilla, "Heat Transfer to Iiquids Boiling under Pressure," Trans. A.I.Ch.E., 41, 1945,755 .

83. Styrikovich, M. A. and G. M. Poliakov, "On the Critical Heat Load with Boiling Liquids in Large Volume," Izv. Alkad. Nauk, Otd. Telkh. Nauk, No. 5, 1948.

84. Kazalcova, E. A., "The Influence of Pressure on the First Crisis in Boiling Water from a Horizontal Surface," in Problems of Heat Transfer with Change of Phase, G.E.I., Koscor, 1953. 
85. McCabe, W. L and J. C. Smith, Unit Operation of Chemical Engineering, McGraw Hill, New York, 1956, 730 .

86. Zuber, N., "Stability of Boiling Heat Transfer," Trans. A.S.M.E., 80, 1958, 711.

87. Zuber, N., "On the Maximum Heat Fluss in Pool Nucleate Boiling of Subcooled Liquids," Memorandum, Dept. of Engr., Univ. of Calif. at Los Angeles, 1957.

88. Zuber, N. and M. Tribus, "Further Remarks on the Stability of Boiling Heat Transfer," Dept. of Engr., University of California at Los Angeles, Rept. 58-5, 1958, al 80 A.E.C. Report A.E.C.U. 3631, 1958.

89. Higbie, R., "The Rate of Absorption of a Gas into a Still Liquid During Short Periods of Exposure," Trans. A.I.Ch.E., 31, 1935, 65.

90. Dankwerts, P. V., "Significance of Liquid-Film Coefficients in Gas Absorption," Ind. Engr. Chem., $43,1951,1460$.

91. Hanratty, T. J., "Turbulent Exchange of Mass and Momentum with a Boundary," A.E.Ch.E. Journal, 2, $1956,359$.

92. Birlchoff, G. and E. H. Zarantonello, Jets, Wakes and Cavities, Academic Press, Nev York, 1958.

93. Taylor, G. I., "The Instability of Liquid Surfaces When Accelerated in a Direction Perpendicular to The ir Plane," Proc. Roy. Soc. London, A-201, 1950, 192.

94. Lewis, D. J., "The Instability of Liquid Surfaces When Accelerated in a Direction Perpendicular to Their Planes II," Proc. Roy. Soc. London, A-202, $1950,81$.

95. Allred, J. C. and G. H. Blount, "Experimental Studies of Taylor Instability," Univ. of Calif. Los Alamos Sc.Lab., Rept. LA-1600, 1954.

96. Bellman, R. and R. H. Pennington, "Effects of Surface Tension on Taylor Instability," Quar. Appl. Math., 12, 1954,151 . 
97. Birlchoff, G. "Taylor Instability and Laminar Mixing," Univ. of Calif. Los Alamos Sc. Lab., Rept. LA-1862, 1955.

98. Birlkhoff, G., "Taylor Instability Appendices to Rept. LA-1862," Univ. of Calif. Los Alamos Sc. Lab., Rept. LA-1927, 1955.

99. Milne-Thompson, L. M., Theoretical Hydrodymamics, Macmillan Co., New York, 1950, 37.

100. Bromley, L., "Heat Transfer in Stable Film Boiling," Chem. Engr. Progress, 46, 1950, 221.

101. Chang, Y. P., "A Theoretical Analysis of Heat Transfer in Natural Convection and in Boiling," A.S.M.E. Trans. 79, 1957, 1501 .

102. Davies, R. M. and G. I. Taylor, "The Mechanism of Large Bubbles Rising Through Extended Liquids and Through Liquids in Tubes," Proc. Roy. Soc. London, A-200, 1949, 375.

103. Garabedien, P. R., "On the Steady-State Bubbles Generated by Taylor Instability," Proc. Roy. Soc. London, A-241, 1957, 423.

104. Birlchoff, G. and D. Carter, "Rising Plane Bubbles," J. of Rat. Mech. and Analysis, 6, 1957, 769.

105. Kutateladze, S. S., Borishanskii, V. M., Novikov, I. I. and 0 . S. Fedjinski, Liquid Metal Heat Transfer, Atomazdat, Muscow, 1958.

106. McAdams, W. H., Heat Transmission (3rd Edition), McGraw Hill, New York, 1954.

107. Lord Rayleigh, Theory of Sound, Dover Publ., New York, 1945.

108. Kazaleova, E. A., "The Influence of Pressure on the First Crisis in Boiling Water," Problems of Heat Transfer During a Change of state, Gosenergoizdat, Moscow, 1953.

109. Kutateladze, S. S, and I. I. Schneiderman, "Esperimental Study of the Influence of the Temperature of a Iiquid on the Change of the Rate of Boiling," Problems of Heat Transfer During a Change of State, 108COW, 1953. 
110. Bartz, D., "Factors Which Influence the Suitabllity of Liquid Propellants as Rocket Motor Regenerative Coolants," Paper presented at the American Rocket Society Spring Meeting, Washington, D. C., April, 1957.

111. Rayleigh, Lord, "Instability of Cylindrical Fluid Surfaces," Philosophical Magazine, 34, Ser. v, $1892,177$.

112. Christiansen, R. M., "Brealcup of a Liquid Jet in a Denser Liquid," Ph.D. Dissertation, University of Pennsylvania, 1955.

113. Christiansen, R. M., and A. N. Hixson, "Breakup of a Liquid Jet in a Denser Liquid," Ind. and Engr. Chemfstry, 49, 1957, 1017.

114. Weber, C. ,"Zum Zerfall eines Filissigkeitsstrahles," Zeitsch. für. angev. Math. und Mech., 1l, Heft 2, $1931,136$. 


\section{APPENDIX A}

The Stability of a Circular Jet

The stability of a cylindrical fluid column under the action of capillary force was analyzed by Rayleigh (106, 111). Considering only the Inertia of the Ifquid Inside the column, the angular frequency of an axially symetric disturbance is given by:

$$
\omega^{2}=\frac{\sigma m R}{\rho^{\prime} R^{3}}\left[(m R)^{2}-1\right] \frac{I_{0}(m R)}{I_{0}(m R)}
$$

where $\rho^{\prime}$ is the density of the fluid within the colum; $m$ is the wave number of the disturbance and $R$ is the radius of the jet. This case would correspond to a liquid jet disintegrating in air. When the inertia of the fluid outside, rather than the fluid inside, the column is important, the angular frequency is given by:

$$
\omega^{2}=\frac{\sigma m R}{\rho R^{3}}\left[1-(m R)^{2}\right] \frac{\stackrel{0}{K}(m R)}{K_{0}(m R)}
$$


where $P$ is the density of the surrounding medium; this case corresponds to the disruption of a jet of alr under water. Since the modified Bessel function of the second kind is given by

$$
K_{0}(z)=\sqrt{\frac{\pi}{2 z}} e^{-z}
$$

It is seen that in both problems the cylindrical column becomes unstable when $m R<1$, i.e., when the wave length $\lambda$ exceeds the circumference of $2 \pi R$ of the jet. The Inertial effects of both jet and surrounding medium were considered by Christiansen $(112,113)$. When the densities of both fluids are taken into account the angular frequency is given by

$$
\omega^{2}=\frac{\sigma m R\left[(m R)^{2}-1\right]}{R^{3}\left[\rho^{\prime} \frac{I_{0}(m R)}{\underline{I}_{0}(m R)}-\rho \frac{K_{0}(m R)}{\dot{K}_{0}(m R)}\right]}
$$

An analysis of the effects of Helmholtz instability upon the disintegration of a liquid jet in air was reported by Weber (114). In his paper the inertia of the jet only was taken into eccount, i.e., the Inertia of the surrounding medium was neglected. In what follows the stability of a cylindrical column will be investigated 
and the Inertial effects of both me dia as well as the effect of Helmholtz Instability will be considered.

Let a cylindrical jet of radius $R$ flow with velocity $u^{\prime}$ in the direction of the vertical $z$ axis. Denote by superscript prime the medium inside the jet. The equation of the disturbed boundary is given by

$$
r=R+\zeta(z, t)
$$

$A-5$

where the disturbance $\zeta$ is of the form

$$
\zeta=\zeta_{0} e^{i(\omega t-m \xi)}
$$

The problem is formulated by considering the equations of continuity for the two flow fields

$$
\begin{aligned}
& \frac{\partial^{2} \phi^{\prime}}{\partial r^{2}}+\frac{1}{r} \frac{\partial \phi^{\prime}}{\partial r}+\frac{\partial^{2} \phi^{\prime}}{\partial z^{2}}=0 \\
& \frac{\partial^{2} \phi}{\partial r^{2}}+\frac{1}{r} \frac{\partial \phi}{\partial r}+\frac{\partial^{2} \phi}{\partial z^{2}}=0
\end{aligned}
$$$$
A-7
$$$$
A-8
$$ 
subject to the boundary conditions

$$
\begin{aligned}
& r=0: \frac{\partial \phi^{\prime}}{\partial r}=0 \\
& r \simeq R:-\frac{\partial \phi^{\prime}}{\partial r}=\frac{d \zeta}{d t} \\
& r \simeq R:-\frac{\partial \phi}{\partial r}=\frac{d \zeta}{d t} \quad A-10 \\
& r \rightarrow \infty: \quad \phi_{A-9} \quad \text { finite } A-11 \\
& r=R: \quad P^{\prime}-P=\sigma\left[\frac{1}{R}+\frac{1}{R_{3}}\right]_{A-12}^{A-13}
\end{aligned}
$$

where $1 / R c$ and $1 / R z$ are the curvature in the transverse and axial sections respectively. From Lamb (Reference 50, Page 473) the sum of the principal curvature is

$$
\frac{1}{R_{c}}+\frac{1}{R_{z}}=\frac{1}{R}-\frac{\xi}{R^{2}}-\frac{\partial^{2} y}{\partial z^{2}}
$$


The pressures $P^{\prime}$ and $P$ satisfy Bernouill1's equation thus:

$$
\begin{aligned}
& \frac{p}{\rho}=\frac{\partial \phi}{\partial t}-\frac{1}{2}\left[\left(\frac{\partial \phi}{\partial z}\right)^{2}+\left(\frac{\partial \phi}{\partial r}\right)^{2}\right]+\text { const. } \\
& \frac{p^{\prime}}{\rho^{\prime}}=\frac{\partial \phi^{\prime}}{\partial t}-\frac{1}{2}\left[\left(u^{\prime}-\frac{\partial \phi^{\prime}}{\partial z}\right)^{2}+\left(\frac{\partial \phi}{\partial r}\right)^{2}\right]+\text { const }
\end{aligned}
$$$$
\text { A-15 }
$$

The solutions of Equations A-7 and A-8 which satisfy the kinematic conditions, i.e., Equations A-9 through A-12, are

$$
\begin{aligned}
& \phi^{\prime}=-i\left(\frac{\omega}{m}-U^{\prime}\right) \frac{I_{0}(m r)}{\dot{I}_{0}(m R)} \zeta_{0} e^{i(\omega t-m z)} \\
& \phi=-i\left(\frac{\omega}{m}\right) \frac{X_{0}(m r)}{\dot{K}_{0}(m R)} \xi_{0} e^{i(\omega t-m z)}
\end{aligned}
$$

The frequency equation is obtained by satisfying the dynamic conditions, i.e., Equations A-13 through A-16 thus 
194

$$
\frac{\sigma\left[(m R)^{2}-1\right]}{R^{2}}=\frac{P^{\prime} I_{0}(m R)}{\dot{I}_{0}(m R)}\left(\frac{\omega}{m}-U^{\prime}\right)^{2} m-\frac{P \frac{K(m R)}{K(m R)}\left(\frac{\omega}{m}\right)^{2} m}{K-19}
$$

We note that for $u^{*}=0$, Equation A-19 is reduced to Equation A-4. With $u^{\prime}=0$ and neglecting the Inertia of the surrounding lIquid, 1.e., $P=0$, Equation A-19 reduces to Equation $A-1$; with $\rho^{\prime}=0$ it reduces to Equation A-2.

Expanding and rearranging Equation A-19 it follows that.

$$
\frac{\omega}{m}=\frac{\rho^{\prime} I_{0}^{*} u^{\prime}}{\rho^{\prime} \Gamma_{0}^{*}-\rho K_{0}^{*}} \pm \frac{\left[\left(\rho^{\prime} \Gamma^{*}-\rho K^{*}\right) W+\rho K^{*} \rho^{\prime} I^{*} u^{\prime 2}\right]^{1 / 2}}{\rho^{\prime} I^{*}-\rho K^{*}}
$$

where

$$
\begin{aligned}
I^{*} & =\frac{I_{0}(m R)}{\dot{I}_{0}(m R)} \\
K^{*} & =\frac{Z_{0}(m R)}{\dot{K_{0}}(m R)} \\
W & =\frac{\sigma}{R^{2}} m\left[(m R)^{2}-1\right]
\end{aligned}
$$

A-21 
The first term on the right-hand side may be called the mean velocity of the flow. Relative to this there are waves travelling with velocities $\pm C$, given by

$$
c^{2}=\frac{\sigma\left[(m R)^{2}-1\right]}{R^{2} m\left[\rho^{\prime} I^{*}-\rho K^{*}\right]}+\frac{\rho^{\prime} I^{*} \rho K^{*} u^{\prime 2}}{\left[\rho^{\prime} I^{*}-\rho K^{*}\right]^{2}}
$$

We note that if the density $\rho$, of the surrounding medium is small. compared to the density $\rho^{\prime}$, of the jet, Equation A-22 becomes

$$
c^{2}=\frac{\sigma\left[(m R)^{2}-1\right]}{R^{2} m\left[\rho^{\prime} I^{*}\right]}+\frac{\rho K^{*}}{\rho^{\prime} I^{*}} u^{\prime 2}
$$

which is the equation derived by Weber. For the present problem the density of the jet $\rho^{\prime}$, is much smaller than the density of the surrounding Iiquid; Equation A-22 can be simplified, thus

$$
c^{2}=\frac{\Gamma}{\rho R} \frac{\left[(m R)^{2}-1\right]}{m R}\left|\frac{\dot{K}_{0}(m R)}{K_{0}(m R)}\right|-\frac{\rho^{\prime} \dot{I}(m R)}{\rho \dot{I}(m R)}\left|\frac{\dot{K}_{0}(m R)}{K_{0}(m R)}\right| U_{A-24}{ }^{2}
$$


For large values of $m R, 1 . e .$, for short waves, Equation A-24 becomes

$$
c^{2}=\frac{\sigma m}{\rho}-\frac{\rho^{\prime} u^{\prime 2}}{\rho}
$$

which is of the same form as Equation VI-I. The condition of stability is that $C$ shall be real. It is seen from Equation A-24 that if $\mathrm{mR}=1$ the motion is unstable for any vapor velocity. The critical condition is then given by

$$
U^{\prime 2}=\frac{\sigma}{\rho^{\prime} R} \frac{\left[(m R)^{2}-l\right]}{m R} \frac{I_{0}^{\prime}(m R)}{\Gamma_{0}(m R)}
$$

With $\mathrm{mR}=2$, Equation A-26 becomes

$$
u^{\cdot 2}=\frac{\sigma}{\rho^{\prime} R}
$$

which is of the same form as Equation VI-5. 